In the format provided by the authors and unedited.

\title{
Global aquifers dominated by fossil groundwaters but wells vulnerable to modern contamination
}

Scott Jasechko, Debra Perrone, Kevin M. Befus, M. Bayani Cardenas, Grant Ferguson, Tom Gleeson, Elco Luijendijk, Jerey J. McDonnell, Richard G. Taylor, Yoshihide Wada and James W. Kirchner 
The Supplementary Information is divided into four sections: S1) compiling global groundwater isotope data, S2) outcomes of young and old groundwater calculations, S3) known biases in our fossil groundwater calculations, and S4) groundwater well construction records in the western USA.

\section{S1. Compiling global groundwater isotope data}

Global groundwater isotope data was compiled from hundreds of primary literature sources and from the United States Geological Survey's Water Quality Portal (www.waterqualitydata.us). The raw data sources are shown in Tables S1 and S2. Compiled groundwater carbon isotope data and their associated aquifer latitude and longitude are tabulated and provided as a supplementary dataset (locations are presented to the nearest degree because some sampling locations are known only to \pm 10 s of kilometres).

Groundwater aquifer systems were delineated using geospatial data from the World-wide Hydrogeological Mapping and Assessment Programme (WHYMAP: www.whymap.org). However, the aquifers delineated by WHYMAP were much larger than the areas explored by individual studies in most cases. Therefore, we delineated the aquifer areas surrounding the groundwater isotope measurement locations reported in individual studies, using WHYMAP aquifer boundaries for guidance where possible.

\section{S2. Outcomes of young and old groundwater calculations}

The set of Figures S7 ( $n=62)$ present the raw, compiled radiochemical $\left({ }^{3} \mathrm{H}\right.$ and ${ }^{14} \mathrm{C}$ activities) and stable isotope $\left({ }^{13} \mathrm{C} /{ }^{12} \mathrm{C}\right.$ and ${ }^{18} \mathrm{O} /{ }^{16} \mathrm{O}$ ratios $)$ data for the study aquifers, as well as the calculated post-1953 and Holocene groundwater fractions. The Holocene groundwater fractions are related to fossil groundwater by [Holocene groundwater fraction] $=1-[$ Fossil groundwater fraction].

The uppermost three panels show profiles of geochemical parameters: tritium activities $\left({ }^{3} \mathrm{H}\right.$; upper left), radiocarbon activities $\left({ }^{14} \mathrm{C}\right.$ of dissolved inorganic carbon; upper middle) and stable carbon isotope compositions $\left(\delta^{13} \mathrm{C}\right.$ of dissolved inorganic carbon; upper right). The carbon 
isotope data are for inorganic carbon species dissolved in the water. The lower three panels present the calculated post-1953 groundwater fractions (lower left), Holocene groundwater fractions (lower middle), and stable oxygen isotope compositions $\left(\delta^{18} \mathrm{O}\right.$; lower right). The stable isotope data are presented in delta notation, where $\delta^{18} \mathrm{O}=\left({ }^{18} \mathrm{O} /{ }^{16} \mathrm{O}\right.$ sample $) /\left({ }^{18} \mathrm{O} /{ }^{16} \mathrm{O}_{\text {standard ocean water }}\right.$ $-1) \times 1000$, and $\delta^{13} \mathrm{C}=\left({ }^{13} \mathrm{C} /{ }^{12} \mathrm{C}_{\text {sample }}\right) /\left({ }^{13} \mathrm{C} /{ }^{12} \mathrm{C}\right.$ Pee Dee Belemnite -1$) \times 1000$.

The two panels presenting post-1953 and Holocene groundwater fractions (lower left and lower middle panels) show multiple points per water sample to convey calculation uncertainties. The large symbols show the midpoint estimate for the calculated post-1953 and Holocene groundwater fraction. Smaller-sized symbols represent the upper and lower (i.e., estimated minimum and estimated maximum) fractions of post-1953 and Holocene groundwater.

For all panels, squares represent the midpoint of the screened (i.e., perforated) interval (or top of screen if the midpoint was not given), whereas diamonds represent the total depth of the well. If information for both the top and the bottom of the well was available, we plotted the point twice, once showing the midpoint depth (squares) and once showing the total depth (diamonds) in order to convey the impact of using total well depths rather than screen midpoint depths for our calculations. Red symbols mark isotope measurements of dissolved inorganic carbon.

\section{S3. Known biases in our fossil groundwater calculations}

Our findings are likely to be conservative estimates of fossil groundwater percentage and young-old mixing. Our results may be affected by a number of biases, the majority of which would tend to bias our results toward lower fossil groundwater fractions; therefore, our first main conclusion - that fossil groundwater dominates global groundwater storage — should likely be at least as strong as it appears to be.

First, our groundwater ${ }^{14} \mathrm{C}$ dataset is necessarily weighted toward permeable sedimentary basins because these tend to have greater groundwater resources and thus more groundwater wells. These permeable basins plausibly support deeper penetration of younger, Holocene-aged waters than less geologically permeable regions where groundwater drilling is rarer and thus groundwater ${ }^{14} \mathrm{C}$ data are less available. Thus, the radiocarbon data used in this analysis may be 
biased towards areas with deeper young groundwater, biasing our calculations toward small fossil groundwater abundances.

Second, recent work has pointed out that groundwater samples exposed to the atmosphere before being measured are vulnerable to contamination by modern atmospheric ${ }^{14} \mathrm{CO}_{2}$, which would bias any such contaminated samples in the direction of smaller apparent fossil groundwater fractions ${ }^{26}$.

Third, widespread irrigation-induced recharge has likely drawn shallower and generally younger groundwaters deeper, plausibly causing our analysis to underestimate fossil groundwater abundance because our dataset is biased toward irrigated regions.

Fourth, groundwater pumping preferentially draws water from more productive geologic strata, which are more likely to host relatively fast-flowing (and consequently younger) groundwaters, again biasing our analysis towards smaller fossil groundwater fractions.

Fifth, it is possible that well pumping could cause upward flows of fossil groundwaters. Among the aforementioned biases, this case is perhaps the most likely to lead us to overestimate fossil groundwater storage because the stored fossil groundwater has been drawn anomalously shallow close to the well.

Most of the potential biases in our analysis lead us to underestimate fossil groundwater storage. Therefore, our estimate that fossil groundwater comprises most (42-85\%) global groundwater storage is likely to be an underestimate. 


\section{S4. Groundwater well construction records in the western USA}

We compile and present well construction depth data compiled from five western states: (i) California, (ii) Colorado, (iii) Kansas, (iv) Nebraska, and (v) Wyoming.

(i) California groundwater well completion report data were obtained by personal communication with a representative from the California Department of Water Resources (www.water.ca.gov; data obtained during March and April, 2016). The California groundwater well construction records are incomplete in the San Joaquin Valley because data for some counties remains unavailable.

(ii) Colorado groundwater well applications data were accessed from cdss.state.co.us/GIS/Pages/AllGISData.aspx (accessed February 21, 2016).

(iii) Kansas water well completion data were obtained by personal communication with a representative from the Kansas Geological Survey (www.kgs.ku.edu; data obtained during March and April, 2016).

(iv) Nebraska registered groundwater well data were accessed from www.ose.state.nm.us/GIS/geospatial_data.php (accessed February 21, 2016).

(v) Wyoming groundwater well construction data were accessed from sites.google.com/a/wyo.gov/seo/documents-data/maps-and-spatial-data (accessed February 21, 2016).

We focus our analysis on wells designed to extract groundwater for use by households, municipalities, industries and food producers. The compiled groundwater well databases include the stated purposes for which the wells were constructed. We retained wells with purposes that suggest the wells were drilled for industrial, household, municipal and agricultural use (Table S3). We removed wells that we could not confirm to have been installed for industrial, household, municipal or agricultural use. 
Table S1. Groundwater isotope data sources

\begin{tabular}{|c|c|c|c|c|c|c|}
\hline Country & Aquifer & Lon. & Lat. & \multicolumn{2}{|c|}{$\begin{array}{c}* \text { Fossil } \\
\text { groundwater } \\
\text { depth }(\mathbf{m})\end{array}$} & Primary source of radiocarbon and tritium data \\
\hline Australia & $\begin{array}{l}\text { Ngalia- } \\
\text { Amadeus }\end{array}$ & 132 & -23 & 15 & 70 & $\begin{array}{l}\text { Cresswell, R., Wischusen, J., Jacobsen, G., Fifield, K. } \\
\text { Assessment of recharge to groundwater systems in the arid } \\
\text { southwestern part of Northern Territory, Australia, using } \\
\text { chlorine-36. Hydrogeology Journal, 7, 393-404 (1999). } \\
\text { Wischusen, J. D. H., Fifield, L. K., Cresswell, R. G. } \\
\text { Hydrogeology of Palm Valley, central Australia; a } \\
\text { Pleistocene flora refuge? Journal of Hydrology, 293, 20-46 } \\
\text { (2004). }\end{array}$ \\
\hline Australia & $\begin{array}{l}\text { Western } \\
\text { Port }\end{array}$ & 145 & -38 & 30 & 75 & $\begin{array}{l}\text { Currell, M., Cendon, D. I., Cheng, X. Analysis of } \\
\text { environmental isotopes in groundwater to understand the } \\
\text { response of a vulnerable coastal aquifer to pumping: Western } \\
\text { Port Basin, south-eastern Australia. Hydrogeology Journal, } \\
\text { 21, 1413-1427 (2013). }\end{array}$ \\
\hline Bangladesh & $\begin{array}{l}\text { Bengal } \\
\text { Basin }\end{array}$ & 91 & 23 & 220 & 250 & $\begin{array}{l}\text { Aggarwal, P. K., Froehlich, K., Basu, A. R., Poreda, R. J., } \\
\text { Kulkarni, K. M., Tarafdar, S. A., Ali, M., Ahmed, N., } \\
\text { Hussain, A., Rahman, M., Ahmed, S. R. Isotope hydrology of } \\
\text { groundwater in Bangladesh: implications for characterization } \\
\text { and mitigation of arsenic in groundwater. IAEA-TC Project } \\
\text { Report: BGD/8/016, International Atomic Energy Agency, } \\
\text { Vienna, 65 pp. (2000). Hoque, M. A., Burgess, W. G. 14C } \\
\text { dating of deep groundwater in the Bengal Aquifer System, } \\
\text { Bangladesh: implications for aquifer anisotropy, recharge } \\
\text { sources and sustainability. Journal of Hydrology, 444, 209- } \\
220 \text { (2012). Klump, S., Kipfer, R., Cirpka, O. A., Harvey, } \\
\text { C. F., Brennwald, M. S., Ashfaque, K. N., Badruzzaman, A. } \\
\text { B. M., Hug, S. J., Imboden, D. M. Groundwater dynamics and } \\
\text { arsenic mobilization in Bangladesh assessed using noble } \\
\text { gases and tritium. Environmental Science and Technology, } \\
\text { 40, 243-250 (2006). Sikdar, P. K., Sahu, P. Understanding } \\
\text { wetland sub-surface hydrology using geologic and isotopic } \\
\text { signatures. Hydrology and Earth System Sciences, 13, 1313- } \\
\text { 1323 (2009). McArthur, J. M., Banerjee, D. M., Sengupta, } \\
\text { S., Ravenscroft, P., Klump, S., Sarkar, A., Dischm B., Kipfer, } \\
\text { R. Migration of As, and }{ }^{3} \text { H/3e ages, in groundwater from } \\
\text { West Bengal: Implications for monitoring. Water Research, } \\
44,4171-4185 \text { (2010). Majumder, R. K., Halim, M. A., } \\
\text { Saha, B. B., Ikawa, R., Nakamura, T., Kagabu, M., Shimada, } \\
\text { J. Groundwater flow system in Bengal Delta, Bangladesh } \\
\text { revealed by environmental isotopes. Environmental Earth } \\
\text { Sciences, 64, 1343-1352 (2011). Dowling, C. B., Poreda, R. } \\
\text { J., Basu, A. R., Peters, S. L., Aggarwal, P. K. Geochemical } \\
\text { study of arsenic release mechanisms in the Bengal Basin } \\
\text { groundwater. Water Resources Research, 38, 1173 (2002). }\end{array}$ \\
\hline Botswana & $\begin{array}{l}\text { Kalahari } \\
\text { Desert }\end{array}$ & 25 & -22 & 150 & 200 & $\begin{array}{l}\text { Stadler, S., Osenbrück, K., Suckow, A. O., Himmelsbach, T., } \\
\text { Hötzl, H. Groundwater flow regime, recharge and regional- } \\
\text { scale solute transport in the semi-arid Kalahari of Botswana } \\
\text { derived from isotope hydrology and hydrochemistry. Journal } \\
\text { of Hydrology, 388, 291-303 (2010). Mazor, E., Verhagen, } \\
\text { B. T., Sellschop, J. P. F, Robins, N. S., Hutton, L. G. Kalahari } \\
\text { groundwaters: their hydrogen, carbon and oxygen isotopes. } \\
\text { International Atomic Energy Proceedings, Vol. 1, } \\
\text { IAEA/CM/182/18, 202-223 (1974). Rahube, T. B. Recharge } \\
\text { and groundwater resources evaluation of the Lokalane- } \\
\text { Ncojane basin (Botswana) using numerical modelling. Thesis, }\end{array}$ \\
\hline
\end{tabular}




\begin{tabular}{|c|c|c|c|c|c|c|}
\hline \multirow[t]{2}{*}{ Country } & \multirow[t]{2}{*}{ Aquifer } & \multirow[t]{2}{*}{ Lon. } & \multirow[t]{2}{*}{ Lat. } & \multicolumn{2}{|c|}{$\begin{array}{c}* \text { Fossil } \\
\text { groundwater } \\
\text { depth }(\mathbf{m})\end{array}$} & \multirow{2}{*}{$\begin{array}{l}\text { Primary source of radiocarbon and tritium data } \\
\text { International Institute for Geo-information Science and Earth } \\
\text { Observation, } 119 \text { pp. (2003). }\end{array}$} \\
\hline & & & & & & \\
\hline China & $\begin{array}{l}\text { Hexi } \\
\text { Corridor } \\
\text { (East) }\end{array}$ & 102 & 39 & 100 & 250 & $\begin{array}{l}\text { Gates, J. B., Edmunds, W. M., Darling, W. G., Ma, J., Pang, } \\
\text { Z., Young, A. A. Conceptual model of recharge to } \\
\text { southeastern Badain Jaran Desert groundwater and lakes from } \\
\text { environmental tracers. Applied Geochemistry, 23, 3519-3534 } \\
\text { (2008). Zhu, G. F., Su, Y. H., Feng, Q. The hydrochemical } \\
\text { characteristics and evolution of groundwater and surface } \\
\text { water in the Heihe River Basin, northwest China. } \\
\text { Hydrogeology Journal, 16, 167-182 (2007). Edmunds, W. } \\
\text { M., Ma, J., Aeschbach-Hertig, W., Kipfer, R., Darbyshire, D. } \\
\text { P. F. Groundwater recharge history and hydrogeochemical } \\
\text { evolution in the Minqin Basin, North West China. Applied } \\
\text { Geochemistry, 21, 2148-2170 (2006). Ma, J., Pan, F., Chen, } \\
\text { L., Edmunds, W. M., Ding, Z., He, J., Zhou, K. Huang, T. } \\
\text { Isotopic and geochemical evidence of recharge sources and } \\
\text { water quality in the Quaternary aquifer beneath Jinchang city, } \\
\text { NW China. Applied Geochemistry, 25, 996-1007 (2010). }\end{array}$ \\
\hline China & $\begin{array}{l}\text { Laizhou } \\
\text { Bay }\end{array}$ & 119 & 37 & 70 & 175 & $\begin{array}{l}\text { Han, D., Kohfahl, C., Song, X., Xiao, G., Yang, J. } \\
\text { Geochemical and isotopic evidence for palaeo-seawater } \\
\text { intrusion into the south coast aquifer of Laizhou Bay, China. } \\
\text { Applied Geochemistry, 26, 863-883 (2011). }\end{array}$ \\
\hline China & $\begin{array}{l}\text { North China } \\
\text { Plain }\end{array}$ & 115 & 38 & 200 & 300 & $\begin{array}{l}\text { Chen Z., Nie, Z., Zhang Z., Qi J., Nan Y. Isotopes and } \\
\text { sustainability of ground water resources, North China Plain. } \\
\text { Groundwater, 43, 485-493 (2005). Kreuzer, A. M., von } \\
\text { Rohden, C., Friedrich, R., Chen, Z., Shi, J., Hajdas, I., Kipfer, } \\
\text { R., Aeschbach-Hertig, W. A record of temperature and } \\
\text { monsoon intensity over the past } 40 \text { kyr from groundwater in } \\
\text { the North China Plain. Chemical Geology, 259, 168-180 } \\
\text { (2009). von Rohden, C., Kreuzer, A., Chen, Z., Kipfer, R., } \\
\text { \& Aeschbach-Hertig, W. Characterizing the recharge regime } \\
\text { of the strongly exploited aquifers of the North China Plain by } \\
\text { environmental tracers. Water Resources Research, 46, } \\
\text { W05511 (2010). Chen, Z., Jixiang, Q., Jianming, X., } \\
\text { Jiaming, X., Hao, Y., Yunju, N. Paleoclimatic interpretation } \\
\text { of the past } 30 \text { ka from isotopic studies of the deep confined } \\
\text { aquifer of the North China plain. Applied Geochemistry, 18, } \\
\text { 997-1009 (2003). }\end{array}$ \\
\hline China & $\begin{array}{l}\text { Songnen } \\
\text { Plain }\end{array}$ & 125 & 49 & 125 & 210 & $\begin{array}{l}\text { Chen, Z., Wei, W., Liu, J., Wang, Y., Chen, J. Identifying the } \\
\text { recharge sources and age of groundwater in the Songnen Plain } \\
\text { (Northeast China) using environmental isotopes. } \\
\text { Hydrogeology Journal, 19, 163-176 (2011). }\end{array}$ \\
\hline Germany & Benkerstein & 11 & 49 & 0 & 120 & $\begin{array}{l}\text { van Geldern, R., Baier, A., Subert, H. L., Kowol, S., Balk, L., } \\
\text { Barth, J. A. C. Pleistocene paleo-groundwater as a pristine } \\
\text { fresh water resource in southern Germany - evidence from } \\
\text { stable and radiogenic isotopes. Science of the Total } \\
\text { Environment, 496, 107-115 (2014). }\end{array}$ \\
\hline Denmark & $\begin{array}{l}\text { Ribe } \\
\text { Formation }\end{array}$ & 9 & 56 & 190 & 290 & $\begin{array}{l}\text { Hinsby, K., Harrar, W. G., Nyegaard, P., Konradi, P. B., } \\
\text { Rasmussen, E. S., Bidstrup, T., Gregersen, U., Boaretto, E. } \\
\text { The Ribe Formation in western Denmark - Holocene and } \\
\text { Pleistocene groundwaters in a coastal Miocene sand aquifer. } \\
\text { Geological Society of London, Special Publications, 189, 29- } \\
48 \text { (2001). }\end{array}$ \\
\hline $\begin{array}{l}\text { Algeria, } \\
\text { Tunisia }\end{array}$ & $\begin{array}{l}\text { Great } \\
\text { Oriental Erg }\end{array}$ & 4 & 32 & 100 & 200 & $\begin{array}{l}\text { Edmunds, W. M., Guendouz, A. H., Mamou, A., Moulla, A., } \\
\text { Shand, P., Zouari, K. Groundwater evolution in the } \\
\text { Continental Intercalaire aquifer of southern Algeria and } \\
\text { Tunisia: trace element and isotopic indicators. Applied } \\
\text { Geochemistry, 18, 805-822 (2003). Guendouz, A., Moulla, }\end{array}$ \\
\hline
\end{tabular}




\begin{tabular}{|c|c|c|c|c|c|c|}
\hline \multirow[t]{2}{*}{ Country } & \multirow[t]{2}{*}{ Aquifer } & \multirow[t]{2}{*}{ Lon. } & \multirow[t]{2}{*}{ Lat. } & \multicolumn{2}{|c|}{$\begin{array}{c}* \text { Fossil } \\
\text { groundwater } \\
\text { depth }(\mathbf{m})\end{array}$} & \multirow[b]{2}{*}{$\begin{array}{l}\text { Primary source of radiocarbon and tritium data } \\
\text { A. S., Edmunds, W. M., Zouari, K., Shand, P., Mamou, A. } \\
\text { Hydrogeochemical and isotopic evolution of water in the } \\
\text { Complexe Terminal aquifer in the Algerian Sahara. } \\
\text { Hydrogeology Journal, 11, 483-495 (2003). Abid, K., } \\
\text { Dulinski, M., Ammar, F. H., Rozanski, K., Zouari, K. } \\
\text { Deciphering interaction of regional aquifers in Southern } \\
\text { Tunisia using hydrochemistry and isotopic tools. Applied } \\
\text { Geochemistry, 27, 44-55 (2012). Abid, K., Zouari, K., } \\
\text { Abidi, B. Identification and characterisation of } \\
\text { hydrogeological relays of continental intercalaire aquifer of } \\
\text { southern Tunisia. Carbonates Evaporites, 25, 65-75 (2010). } \\
\text { Abid, K., Ammar, F. H., Chkir, N., Zouari, K. Relationship } \\
\text { between Senonian and deep aquifers in Southern Tunisia. } \\
\text { Quaternary International, 257, 13-26 (2012). }\end{array}$} \\
\hline & & & & & & \\
\hline Estonia & $\begin{array}{l}\text { Cambrian- } \\
\text { Vendian }\end{array}$ & 27 & 58 & 40 & 50 & $\begin{array}{l}\text { Vaikmäe, R., Vallner, L., Loosli, H. H., Blaser, P. C., } \\
\text { Juillard-Tardent, M. Palaeogroundwater of glacial origin in } \\
\text { the Cambrian-Vendian aquifer of northern Estonia. } \\
\text { Geological Society, London, Special Publications, 189, 17-27 } \\
\text { (2001). }\end{array}$ \\
\hline France & $\begin{array}{l}\text { Aquitaine } \\
\text { Basin }\end{array}$ & 0 & 46 & 80 & 180 & $\begin{array}{l}\text { Jiráková, H., Huneau, F., Celle-Jeanton, H., Hrkal, Z., Le } \\
\text { Coustumer, P. Palaeorecharge conditions of the deep aquifers } \\
\text { of the Northern Aquitaine region (France). Journal of } \\
\text { Hydrology, 368, 1-16 (2009). }\end{array}$ \\
\hline France & $\begin{array}{l}\text { Bathonian- } \\
\text { Bajocian }\end{array}$ & 0 & 49 & 20 & 55 & $\begin{array}{l}\text { Barbecot, F., Marlin, C., Gibert, E., Dever, L. Hydrochemical } \\
\text { and isotopic characterisation of the Bathonian and Bajocian } \\
\text { coastal aquifer of the Caen area (northern France). Applied } \\
\text { Geochemistry, 15, 791-805 (2000). }\end{array}$ \\
\hline France & $\begin{array}{l}\text { Lorraine } \\
\text { Sandstone }\end{array}$ & 7 & 48 & 120 & 320 & $\begin{array}{l}\text { Celle-Jeanton, H. Huneau, F., Travi, Y., Edmunds, W. M. } \\
\text { Twenty years of groundwater evolution in the Triassic } \\
\text { sandstone aquifer of Lorraine: Impacts on baseline water } \\
\text { quality. Applied Geochemistry, 24, 1198-1213 (2009). }\end{array}$ \\
\hline Hungary & $\begin{array}{l}\text { Great } \\
\text { Hungarian } \\
\text { Plain }\end{array}$ & 21 & 47 & 80 & 250 & $\begin{array}{l}\text { Stute, M., Deák, J. Environmental isotope study }{ }^{14} \mathrm{C},{ }^{13} \mathrm{C},{ }^{18} \mathrm{O} \text {, } \\
\mathrm{D} \text {, noble gases on deep groundwater circulation systems in } \\
\text { Hungary with reference to paleoclimate. Radiocarbon, } 31 \text {, } \\
902-918 \text { (1989). }\end{array}$ \\
\hline Hungary & $\begin{array}{l}\text { Pannonian } \\
\text { Basin }\end{array}$ & 20 & 46 & 160 & 300 & $\begin{array}{l}\text { Varsányi, I., Palcsu, L., Kovács, L. Ó. Groundwater flow } \\
\text { system as an archive of palaeotemperature: noble gas, } \\
\text { radiocarbon, stable isotope and geochemical study in the } \\
\text { Pannonian Basin, Hungary. Applied Geochemistry, 26, 91- } \\
104 \text { (2011). Szocs, T., Rman, N., Süveges, M., Palcsu, L., } \\
\text { Tóth, G., Lapanje, A. The application of isotope and chemical } \\
\text { analyses in managing transboundary groundwater resources. } \\
\text { Applied Geochemistry, 32, 95-107 (2013). }\end{array}$ \\
\hline Indonesia & $\begin{array}{l}\text { Jakarta } \\
\text { Basin }\end{array}$ & 107 & -6 & 50 & 135 & $\begin{array}{l}\text { Geyh, M. A., Sofner, B. Groundwater analysis of } \\
\text { environmental carbon and other isotopes from the Jakarta } \\
\text { Basin Aquifer, Indonesia. Radiocarbon, 31, 919-925 (1989). } \\
\text { Kagabu, M., Shimada, J., Delinom, R., Tsujimura, M., } \\
\text { Taniguchi, M. Groundwater flow system under a rapidly } \\
\text { urbanizing coastal city as determined by hydrogeochemistry. } \\
\text { Journal of Asian Earth Sciences, 40, 226-239 (2011). } \\
\text { Kagabu, M., Shimada, J., Delinom, R., Nakamura, T., } \\
\text { Taniguchi, M. Groundwater age rejuvenation caused by } \\
\text { excessive urban pumping in Jakarta area, Indonesia. } \\
\text { Hydrogeological Processes, 27, 2591-2604 (2013). }\end{array}$ \\
\hline Israel & $\begin{array}{l}\text { Arava } \\
\text { Valley }\end{array}$ & 35 & 31 & 80 & 250 & $\begin{array}{l}\text { Vengosh, A., Hening, S., Ganor, J., Mayer, B., } \\
\text { Weyhenmeyer, C. E., Bullen, T. D., Paytan, A. New isotopic } \\
\text { evidence for the origin of groundwater from the Nubian } \\
\text { Sandstone Aquifer in the Negev, Israel. Applied }\end{array}$ \\
\hline
\end{tabular}




\begin{tabular}{|c|c|c|c|c|c|c|}
\hline \multirow[t]{2}{*}{ Country } & \multirow[t]{2}{*}{ Aquifer } & \multirow[t]{2}{*}{ Lon. } & \multirow[t]{2}{*}{ Lat. } & \multicolumn{2}{|c|}{$\begin{array}{c}* \text { Fossil } \\
\text { groundwater } \\
\text { depth }(\mathbf{m})\end{array}$} & \multirow{2}{*}{$\begin{array}{l}\text { Primary source of radiocarbon and tritium data } \\
\text { Geochemistry, 22, 1052-1073 (2007). Burg, A., } \\
\text { Zilberbrand, M., Yechieli, Y. Radiocarbon variability in } \\
\text { groundwater in an extremely arid zone- - the Arava Valley, } \\
\text { Israel. Radiocarbon, 55, 963-978 (2013). }\end{array}$} \\
\hline & & & & & & \\
\hline India & $\begin{array}{l}\text { Tiruvadanai } \\
\text { Aquifer }\end{array}$ & 79 & 10 & 130 & 280 & $\begin{array}{l}\text { Kumar, U. S., Sharma, S., Navada, S. V., Deodhar, A. S. } \\
\text { Environmental isotopes investigation on recharge processes } \\
\text { and hydrodynamics of the coastal sedimentary aquifers of } \\
\text { Tiruvadanai, Tamilnadu State, India. Journal of Hydrology, } \\
\text { 364, 23-39 (2009). }\end{array}$ \\
\hline Italy & $\begin{array}{l}\text { Emilia } \\
\text { Romagna } \\
\text { Plain }\end{array}$ & 11 & 45 & 110 & 300 & $\begin{array}{l}\text { Gorgni, C., Martinelli, G., Sighinolfi, G. P. Isotopic evidence } \\
\text { of paleowaters in the Po sedimentary basin (Northern Italy). } \\
\text { Geochemical Journal, 16, 51-61 (1982). Martinelli, G., } \\
\text { Chahoud, A., Dadomo, A., Fava, A. Isotopic features of } \\
\text { Emilia-Romagna region (North Italy) groundwaters: } \\
\text { Environmental and climatological implications. Journal of } \\
\text { Hydrology, 519, 1928-1938 (2014). Mayer, A., Sültenfuß, } \\
\text { J., Travi, Y., Rebeix, R., Purtschert, R., Claude, C., Le Gal La } \\
\text { Salle, C., Miche, H., Conchetto, E. A multi-tracer study of } \\
\text { groundwater origin and transit-time in the aquifers of the } \\
\text { Venice region (Italy). Applied Geochemistry, 50, 177-198 } \\
\text { (2014). }\end{array}$ \\
\hline Japan & $\begin{array}{l}\text { Kimitsu- } \\
\text { Yoro } \\
\text { Aquifer }\end{array}$ & 140 & 35 & 360 & 440 & $\begin{array}{l}\text { Tosaki, Y., Tase, N., Kondoh, A., Kimikazu, S., Takahasi, T., } \\
\text { Nagashima, Y. Distribution of } 36 \mathrm{Cl} \text { in the Yoro River Basin, } \\
\text { Central Japan, and its relation to the residence time of the } \\
\text { regional groundwater flow system. Water, } 3,64-78 \text { (2011). } \\
\text { Machida, I., Suzuki, Y., Takeuchi, M. Carbon-14 age and } \\
\text { chemical evolution of Ca(HCO }\left(\mathrm{HCO}_{3}\right)_{2} \text {-type groundwater of age } \\
\text { less than 8,000 years in a confined sandy and muddy } \\
\text { Pleistocene aquifer, Japan. Hydrogeology Journal, 21, 1289- } \\
1305 \text { (2013). }\end{array}$ \\
\hline Libya & Kufra Basin & 23 & 24 & 20 & 450 & $\begin{array}{l}\text { Al Faitouri, M., Sanford, W. E. Stable and radio-isotope } \\
\text { analysis to determine recharge timing and paleoclimate of } \\
\text { sandstone aquifers in central and southeast Libya. } \\
\text { Hydrogeology Journal, DOI 10.1007/s10040-015-1232-7, } \\
\text { (2015). }\end{array}$ \\
\hline Libya & Sirte Basin & 22 & 28 & 20 & 60 & $\begin{array}{l}\text { Edmunds, W. M., Wright, E. P. Groundwater recharge and } \\
\text { palaeoclimate in the Sirte and Kufra basins, Libya. Journal of } \\
\text { Hydrology, 40, 215-241 (1979). }\end{array}$ \\
\hline Nigeria & Chad Basin & 14 & 11 & 0 & 275 & $\begin{array}{l}\text { Maduabuchi, C., Faye, S., Maloszewski, P. Isotope evidence } \\
\text { of palaeorecharge and palaeoclimate in the deep confined } \\
\text { aquifers of the Chad Basin, NE Nigeria. Science of the Total } \\
\text { Environment, 370, 467-479 (2006). }\end{array}$ \\
\hline Poland & $\begin{array}{l}\text { Malm } \\
\text { Limestones }\end{array}$ & 20 & 50 & 40 & 100 & $\begin{array}{l}\text { Zuber, A., Weise, S. M., Motyka, J., Osenbrück, K., } \\
\text { Różański, K. Age and flow pattern of groundwater in a } \\
\text { Jurassic limestone aquifer and related Tertiary sands derived } \\
\text { from combined isotope, noble gas and chemical data. Journal } \\
\text { of Hydrology, 286, 87-112 (2004). }\end{array}$ \\
\hline Poland & $\begin{array}{l}\text { Mazovian } \\
\text { Basin }\end{array}$ & 21 & 52 & 0 & 175 & $\begin{array}{l}\text { Zuber, A., Weise, S. M., Osenbrück, Pajnowska, H., } \\
\text { Grabczak, J. Age and recharge pattern of water in the } \\
\text { Oligocene of the Mazovian basin (Poland) as indicated by } \\
\text { environmental tracers. Journal of Hydrology, 233, 174-188 } \\
\text { (2000). }\end{array}$ \\
\hline Poland & $\begin{array}{l}\text { Southern } \\
\text { Carbonates }\end{array}$ & 21 & 50 & 160 & 190 & $\begin{array}{l}\text { Zuber, A., Weise, S. M., Osenbriick, K., Mateńko, T. Origin } \\
\text { and age of saline waters in Busko Spa (Southern Poland) } \\
\text { determined by isotope, noble gas and hydrochemical } \\
\text { methods: evidence of interglacial and pre-Quaternary warm } \\
\text { climate recharges. Applied Geochemistry, 12, 643-660 } \\
\text { (1997). Samborska, K., Różkowski, A., Małoszewski, P. }\end{array}$ \\
\hline
\end{tabular}




\begin{tabular}{|c|c|c|c|c|c|c|}
\hline \multirow[t]{2}{*}{ Country } & \multirow[t]{2}{*}{ Aquifer } & \multirow[t]{2}{*}{ Lon. } & \multirow[t]{2}{*}{ Lat. } & \multicolumn{2}{|c|}{$\begin{array}{c}* \text { Fossil } \\
\text { groundwater } \\
\text { depth }(\mathbf{m})\end{array}$} & \multirow{2}{*}{$\begin{array}{l}\text { Primary source of radiocarbon and tritium data } \\
\text { Estimation of groundwater residence time using } \\
\text { environmental radioisotopes }\left({ }^{14} \mathrm{C}, \mathrm{T}\right) \text { in carbonate aquifers, } \\
\text { southern Poland. Isotopes in environmental and health } \\
\text { studies, 49, 73-97 (2012). }\end{array}$} \\
\hline & & & & & & \\
\hline Senegal & $\begin{array}{l}\text { Diass } \\
\text { Aquifer }\end{array}$ & -15 & 16 & 50 & 300 & $\begin{array}{l}\text { Castany, G., Marce, A., Margat, J., Moussu, H., Vuillaume, } \\
\text { Y., Evin, J. An environmental isotope study of the } \\
\text { groundwater regime in large aquifers. in: Isotope techniques } \\
\text { in groundwater hydrology 1974, Vol. I., International Atomic } \\
\text { Energy Agency, Vienna, Austria, 243-256 (1974). } \\
\text { Madioune, D. H., Faye, S., Orban, P., Brouyère, S., } \\
\text { Dassargues, A., Mudry, J., Stumpp, C., Maloszewski, P. } \\
\text { Application of isotopic tracers as a tool for understanding } \\
\text { hydrodynamic behavior of the highly exploited Diass aquifer } \\
\text { system (Senegal). Journal of Hydrology, 511, 443-459 (2014). }\end{array}$ \\
\hline Thailand & $\begin{array}{l}\text { Bangkok } \\
\text { Basin }\end{array}$ & 101 & 14 & 60 & 120 & $\begin{array}{l}\text { Sanford, W., Buapeng, S. Assessment of a groundwater flow } \\
\text { model of the Bangkok Basin, Thailand, using carbon-14- } \\
\text { based ages and paleohydrology. Hydrogeology Journal, 4, 26- } \\
40 \text { (1996). }\end{array}$ \\
\hline Tunisia & Djérid Basin & 8 & 34 & 30 & 120 & $\begin{array}{l}\text { Kamel, S. Dassi, L., Zouari, K. Approche hydrogéologique et } \\
\text { hydrochimique des échanges hydrodynamiques entre } \\
\text { aquifères profond et superficiel du bassin du Djérid, Tunisie. } \\
\text { Hydrological Sciences Journal, 51, 713-730 (2006). }\end{array}$ \\
\hline Turkey & $\begin{array}{l}\text { Kazan } \\
\text { Trona }\end{array}$ & 40 & 33 & 20 & 70 & $\begin{array}{l}\text { Arslan, S., Yazicigil, H., Stute, M., Schlosser, P. } \\
\text { Environmental isotopes and noble gases in the deep aquifer } \\
\text { system of Kazan Trona Ore Field, central Turkey and links to } \\
\text { paleoclimate. Quaternary Research, 79, 292-303 (2012). }\end{array}$ \\
\hline USA & $\begin{array}{l}\text { California } \\
\text { Bay: East }\end{array}$ & -122 & 38 & 125 & 200 & Water Quality Portal (www.waterqualitydata.us) \\
\hline USA & $\begin{array}{l}\text { California } \\
\text { Bay: } \\
\text { Monterey }\end{array}$ & -122 & 37 & 190 & 250 & Water Quality Portal (www.waterqualitydata.us) \\
\hline USA & $\begin{array}{l}\text { Cambrian- } \\
\text { Ordovician }\end{array}$ & -88 & 42 & 260 & 400 & Water Quality Portal (www.waterqualitydata.us) \\
\hline USA & $\begin{array}{l}\text { Castle- } \\
\text { Hayne } \\
\text { Aquifer }\end{array}$ & -78 & 35 & 55 & 80 & $\begin{array}{l}\text { Kennedy, C.D., Genereux, D.P. }{ }^{14} \text { C groundwater age and the } \\
\text { importance of chemical fluxes across aquifer boundaries in } \\
\text { confined Cretaceous aquifers of North Carolina, USA. } \\
\text { Radiocarbon, } 49,1181-1203 \text { (2007). Water Quality Portal } \\
\text { (www.waterqualitydata.us) }\end{array}$ \\
\hline USA & $\begin{array}{l}\text { Columbia } \\
\text { Basin }\end{array}$ & -119 & 47 & 50 & 350 & $\begin{array}{l}\text { Brown, K. B., McIntosh, J. C., Rademacher, L. K., Lohse, K. } \\
\text { A. Impacts of agricultural irrigation recharge on groundwater } \\
\text { quality in a basalt aquifer system (Washington, USA): a } \\
\text { multi-tracer approach. Hydrogeology Journal, 19, 1039-1051 } \\
\text { (2011). Water Quality Portal (www.waterqualitydata.us) }\end{array}$ \\
\hline USA & $\begin{array}{l}\text { Denver } \\
\text { Basin }\end{array}$ & -105 & 39 & 125 & 200 & Water Quality Portal (www.waterqualitydata.us) \\
\hline USA & $\begin{array}{l}\text { Eureka } \\
\text { Coast }\end{array}$ & -124 & 41 & 70 & 120 & Water Quality Portal (www.waterqualitydata.us) \\
\hline USA & $\begin{array}{l}\text { Floridan } \\
\text { Aquifer }\end{array}$ & -82 & 33 & 90 & 140 & $\begin{array}{l}\text { Clark, J. F., Stute, M., Schlosser, P., Drenkard, S., Bonani, G. } \\
\text { A tracer study of the Floridan aquifer in southeastern Georgia: } \\
\text { Implications for groundwater flow and paleoclimate. Water } \\
\text { Resources Research, 33, 281-289 (1997). Water Quality } \\
\text { Portal (www.waterqualitydata.us) }\end{array}$ \\
\hline USA & $\begin{array}{l}\text { Central } \\
\text { High Plains }\end{array}$ & -103 & 37 & 120 & 190 & $\begin{array}{l}\text { Clark, J. F., Davisson, M. L., Hudson, G. B., Macfarlane, P. } \\
\text { A. Noble gases, stable isotopes, and radiocarbon as tracers of } \\
\text { flow in the Dakota aquifer, Colorado and Kansas. Journal of } \\
\text { Hydrology, 211, 151-167 (1998). Dutton, A. R. } \\
\text { Groundwater isotopic evidence for paleorecharge in US High } \\
\text { Plains aquifers. Quaternary Research, 43, 221-231 (1995). }\end{array}$ \\
\hline
\end{tabular}




\begin{tabular}{|c|c|c|c|c|c|c|}
\hline \multirow[t]{2}{*}{ Country } & \multirow[t]{2}{*}{ Aquifer } & \multirow[t]{2}{*}{ Lon. } & \multirow[t]{2}{*}{ Lat. } & \multicolumn{2}{|c|}{$\begin{array}{c}* \text { Fossil } \\
\text { groundwater } \\
\text { depth }(\mathbf{m})\end{array}$} & \multirow{2}{*}{$\begin{array}{l}\text { Primary source of radiocarbon and tritium data } \\
\text { McMahon, P. B., Böhlke, J. K., Christenson, S. C. } \\
\text { Geochemistry, radiocarbon ages, and paleorecharge } \\
\text { conditions along a transect in the central High Plains aquifer, } \\
\text { southwestern Kansas, USA. Applied Geochemistry, 19, 1655- } \\
1686 \text { (2004). Water Quality Portal } \\
\text { (www.waterqualitydata.us) }\end{array}$} \\
\hline & & & & & & \\
\hline USA & $\begin{array}{l}\text { North High } \\
\text { Plains }\end{array}$ & -104 & 42 & 170 & 240 & $\begin{array}{l}\text { McMahon, P. B., Böhlke, J. K., Carney, C. P. Vertical } \\
\text { Gradients in Water Chemistry and Age in the Northern High } \\
\text { Plains Aquifer, Nebraska, 2003. US Geological Survey } \\
\text { Scientific Investigations Report 2006-5294 (2006). Water } \\
\text { Quality Portal (www.waterqualitydata.us) }\end{array}$ \\
\hline USA & $\begin{array}{l}\text { South High } \\
\text { Plains }\end{array}$ & -102 & 36 & 120 & 275 & Water Quality Portal (www.waterqualitydata.us) \\
\hline USA & $\begin{array}{l}\text { Houston } \\
\text { Coast (TX) }\end{array}$ & -95 & 29 & 20 & 140 & Water Quality Portal (www.waterqualitydata.us) \\
\hline USA & $\begin{array}{l}\text { Jacksonville } \\
\text { Aquifer }\end{array}$ & -82 & 30 & 55 & 70 & Water Quality Portal (www.waterqualitydata.us) \\
\hline USA & $\begin{array}{l}\text { Livermore } \\
\text { Valley }\end{array}$ & -122 & 38 & 200 & 220 & Water Quality Portal (www.waterqualitydata.us) \\
\hline USA & $\begin{array}{l}\text { Los Angeles } \\
\text { Basin }\end{array}$ & -118 & 34 & 360 & 550 & $\begin{array}{l}\text { Swarzenski, P. W., Baskaran, M., Rosenbauer, R. J., } \\
\text { Edwards, B. D., Land, M. A combined radio-and stable- } \\
\text { isotopic study of a California coastal aquifer system. Water, } \\
\text { 5, 480-504 (2013). Water Quality Portal } \\
\text { (www.waterqualitydata.us) }\end{array}$ \\
\hline USA & $\begin{array}{l}\text { Tertiary } \\
\text { Aquifer } \\
\text { (MT) }\end{array}$ & -105 & 47 & 20 & 55 & Water Quality Portal (www.waterqualitydata.us) \\
\hline USA & $\begin{array}{l}\text { Mahomet } \\
\text { Aquifer }\end{array}$ & -89 & 40 & 100 & 460 & $\begin{array}{l}\text { Hackley, K. C., Panno, S. V., Anderson, T. F. Chemical and } \\
\text { isotopic indicators of groundwater evolution in the basal } \\
\text { sands of a buried bedrock valley in the midwestern United } \\
\text { States: Implications for recharge, rock-water interactions, and } \\
\text { mixing. Geological Society of America Bulletin, 122, 1047- } \\
1066 \text { (2010). Water Quality Portal } \\
\text { (www.waterqualitydata.us) }\end{array}$ \\
\hline USA & $\begin{array}{l}\text { Aquia- } \\
\text { Patapsco } \\
\text { Aquifer }\end{array}$ & -77 & 39 & 25 & 75 & $\begin{array}{l}\text { Aeschbach-Hertig, W., Stute, M., Clark, J. F., Reuter, R. F., } \\
\text { Schlosser, P. A paleotemperature record derived from } \\
\text { dissolved noble gases in groundwater of the Aquia Aquifer } \\
\text { (Maryland, USA). Geochimica et Cosmochimica Acta, 66, } \\
\text { 797-817 (2002). Plummer, L. N., Eggleston, J. R., } \\
\text { Andreasen, D. C., Raffensperger, J. P., Hunt, A. G., Castle, G. } \\
\text { C. Old groundwater in parts of the upper Patapsco aquifer, } \\
\text { Atlantic Coastal Plain, Maryland, USA: evidence from } \\
\text { radiocarbon, chlorine-36 and helium-4. Hydrogeology } \\
\text { Journal, 20, 1269-1294 (2012). Water Quality Portal } \\
\text { (www.waterqualitydata.us) }\end{array}$ \\
\hline USA & $\begin{array}{l}\text { Mississippi } \\
\text { Alluvial }\end{array}$ & -91 & 33 & 105 & 135 & Water Quality Portal (www.waterqualitydata.us) \\
\hline USA & $\begin{array}{l}\text { Mississippi } \\
\text { Embayment }\end{array}$ & -93 & 33 & 45 & 115 & Water Quality Portal (www.waterqualitydata.us) \\
\hline USA & $\begin{array}{l}\text { Mississippi } \\
\text { Embayment: } \\
\text { Memphis }\end{array}$ & -90 & 35 & 195 & 510 & $\begin{array}{l}\text { Larsen, D., Gentry, R. W., Solomon, D. K. The geochemistry } \\
\text { and mixing of leakage in a semi-confined aquifer at a } \\
\text { municipal well field, Memphis, Tennessee, USA. Applied } \\
\text { geochemistry, 18, 1043-1063 (2003). Water Quality Portal } \\
\text { (www.waterqualitydata.us) }\end{array}$ \\
\hline USA & $\begin{array}{l}\text { Atlantic } \\
\text { Coast }\end{array}$ & -77 & 36 & 40 & 80 & Water Quality Portal (www.waterqualitydata.us) \\
\hline USA & $\begin{array}{l}\text { Palouse } \\
\text { Basin }\end{array}$ & -117 & 47 & 60 & 80 & $\begin{array}{l}\text { Douglas, A., Osiensky, J. L., Keller, C. K. Carbon-14 dating } \\
\text { of ground water in the Palouse Basin of the Columbia river }\end{array}$ \\
\hline
\end{tabular}




\begin{tabular}{|c|c|c|c|c|c|c|}
\hline \multirow[t]{2}{*}{ Country } & \multirow[t]{2}{*}{ Aquifer } & \multirow[t]{2}{*}{ Lon. } & \multirow[t]{2}{*}{ Lat. } & \multicolumn{2}{|c|}{$\begin{array}{c}* \text { Fossil } \\
\text { groundwater } \\
\text { depth }(\mathbf{m})\end{array}$} & \multirow{2}{*}{$\begin{array}{l}\text { Primary source of radiocarbon and tritium data } \\
\begin{array}{l}\text { basalts. Journal of Hydrology, 334, 502-512 (2007). Water } \\
\text { Quality Portal (www.waterqualitydata.us) }\end{array} \\
\end{array}$} \\
\hline & & & & & & \\
\hline USA & $\begin{array}{l}\text { San Diego } \\
\text { Basin }\end{array}$ & -117 & 33 & 180 & 250 & Water Quality Portal (www.waterqualitydata.us) \\
\hline USA & $\begin{array}{l}\text { Silurian- } \\
\text { Devonian } \\
\text { (IL) }\end{array}$ & -88 & 41 & 30 & 430 & Water Quality Portal (www.waterqualitydata.us) \\
\hline USA & $\begin{array}{l}\text { South } \\
\text { Central } \\
\text { Valley (CA) }\end{array}$ & -121 & 38 & 240 & 280 & $\begin{array}{l}\text { Jurgens, B. C., Burow, K. R., Dalgish, B. A., Shelton, J. L. } \\
\text { Hydrogeology, water chemistry, and factors affecting the } \\
\text { transport of contaminants in the zone of contribution of a } \\
\text { public-supply well in Modesto, eastern San Joaquin Valley, } \\
\text { California. Scientific Investigations Report 2008-5156 } \\
\text { (2008). Water Quality Portal (www.waterqualitydata.us) }\end{array}$ \\
\hline USA & $\begin{array}{l}\text { Surficial } \\
\text { Aquifers }\end{array}$ & -81 & 27 & 60 & 110 & $\begin{array}{l}\text { Morrissey, S. K., Clark, J. F., Bennett, M., Richardson, E., } \\
\text { Stute, M. Groundwater reorganization in the Floridan aquifer } \\
\text { following Holocene sea-level rise. Nature Geoscience, 3, 683- } \\
687 \text { (2010). Water Quality Portal } \\
\text { (www.waterqualitydata.us) }\end{array}$ \\
\hline USA & Tampa Bay & -82 & 27 & 25 & 50 & Water Quality Portal (www.waterqualitydata.us) \\
\hline USA & $\begin{array}{l}\text { Temecula- } \\
\text { Murrieta } \\
\text { Basin }\end{array}$ & -117 & 33 & 305 & 330 & Water Quality Portal (www.waterqualitydata.us) \\
\hline USA & $\begin{array}{l}\text { Wharton } \\
\text { Tract }\end{array}$ & -75 & 40 & 70 & 140 & $\begin{array}{l}\text { Thompson, G. M., Hayes, J. M. Trichlorofluoromethane in } \\
\text { groundwater-a possible tracer and indicator of groundwater } \\
\text { age. Water Resources Research, 15, 546-554 (1979). Water } \\
\text { Quality Portal (www.waterqualitydata.us) }\end{array}$ \\
\hline
\end{tabular}

* the depth to fossil groundwater below the land surface. A shallow and deep estimate are provided. The shallower depth to fossil groundwater (left column under heading "Fossil groundwater (m)") specifies the depth below which the majority $(>50 \%)$ of well water samples from a given aquifer must contain some fraction of fossil groundwater (i.e., over half the samples have a minimum fossil groundwater fraction of greater than zero). The deeper depth to fossil groundwater (right column under heading "Fossil groundwater (m)") represents a depth below which the majority $(>50 \%)$ of sampled well waters from a given aquifer system contain mostly fossil groundwater (i.e., over half of the samples deeper than the depth have a minimum fossil groundwater fraction of greater than 0.5 ). 
Table S2. Groundwater isotope data sources outside of the study aquifers

\begin{tabular}{|c|c|}
\hline \# & Reference \\
\hline 1 & $\begin{array}{l}\text { Abouelmagd, A., Sultan, M., Sturchio, N. C., Soliman, F., Rashed, M., Ahmed, M., Kehew, A. E., Milewski, A., } \\
\text { Chouinard, K. Paleoclimate record in the Nubian Sandstone Aquifer, Sinai Peninsula, Egypt. Quaternary Research, 81, } \\
\text { 158-167 (2014). }\end{array}$ \\
\hline 2 & $\begin{array}{l}\text { Adiaffi, B., Marlin, C., Oga, Y. M. S., Massault, M., Noret, A., Biemi, J. Palaeoclimatic and deforestation effect on the } \\
\text { coastal fresh groundwater resources of SE Ivory Coast from isotopic and chemical evidence. Journal of Hydrology, } \\
369,130-141(2009) \text {. }\end{array}$ \\
\hline 3 & $\begin{array}{l}\text { Ahmed, M. A., Abdel Samie, S. G., El-Maghrabi, H. M. Recharge and contamination sources of shallow and deep } \\
\text { groundwater of Pleistocene aquifer in El-Sadat industrial city: Isotope and hydrochemical approaches. Environmental } \\
\text { Earth Science, 62, 751-768 (2011). }\end{array}$ \\
\hline 4 & $\begin{array}{l}\text { Ako Ako, A., Shimada, J., Hosono, T., Ichiyanagi, K., Nkeng, G. E., Eyong, G. E. T., Roger, N. N. Hydrogeochemical } \\
\text { and isotopic characteristics of groundwater in Mbanga, Njombe and Penja (Banana Plain) - Cameroon. Journal of } \\
\text { African Earth Sciences, 75, 25-36 (2012). }\end{array}$ \\
\hline 5 & $\begin{array}{l}\text { Al-Charideh, A., Abou-Zakhem, B. Geochemical and isotopic characterization of groundwater from the Paleogene } \\
\text { limestone aquifer of the Upper Jezireh, Syria. Environmental Earth Sciences, 59, 1065-1078 (2010). }\end{array}$ \\
\hline 6 & $\begin{array}{l}\text { Al-Charideh, A., Hasan, A. Use of isotopic tracers to characterize the interaction of water components and nitrate } \\
\text { contamination in the arid Rasafeh area (Syria). Environmental Earth Sciences, 70, 71-82 (2013). }\end{array}$ \\
\hline 7 & $\begin{array}{l}\text { Al-Charideh, A., Kattan, B. Isotope hydrology of deep groundwater in Syria: renewable and non-renewable } \\
\text { groundwater and paleoclimate impact. Hydrogeology Journal, 24, 79-98 (2016). }\end{array}$ \\
\hline 8 & $\begin{array}{l}\text { Al-Katheeri, E. S., Howari, F. M., Murad, A. A. Hydrogeochemistry and pollution assessment of quaternary-tertiary } \\
\text { aquifer in the Liwa area, United Arab Emirates. Environmental Earth Sciences, 59, 581-592 (2009). }\end{array}$ \\
\hline 9 & $\begin{array}{l}\text { Alvarado, J. C., Pačes, T., Purtschert, R. Dating groundwater in the Bohemian Cretaceous Basin: Understanding tracer } \\
\text { variations in the subsurface. Applied Geochemistry, 29, 189-198 (2013). }\end{array}$ \\
\hline 10 & $\begin{array}{l}\text { Aravena, R., Wassenaar, L. I., Plummer, L. N. Estimating 14C groundwater ages in a methanogenic aquifer. Water } \\
\text { Resources Research, 31, 2307-2317 (1995). }\end{array}$ \\
\hline 11 & $\begin{array}{l}\text { Atkinson, A. P., Cartwright, I., Gilfedder, B. S., Cendon, D. I., Unland, N. P., Hofmann, H. Using 14C and 3H to } \\
\text { understand groundwater flow and recharge in an aquifer window. Hydrology and Earth System Sciences, 18, 4951- } \\
4964 \text { (2014). }\end{array}$ \\
\hline 12 & $\begin{array}{l}\text { Awad, M. A., Hammad, F. A., Aly, A. I. M., Sadek, M. A. Use of environmental isotopes and hydrochemistry as } \\
\text { indicators for the origin of groundwater resources in El Dabaa area, northwestern coastal zone of Egypt. Environmental } \\
\text { Geochemistry and Health, 16, 31-38 (1994). }\end{array}$ \\
\hline 13 & $\begin{array}{l}\text { Bajjali, W. Recharge mechanism and hydrochemistry evaluation of groundwater in the Nuaimeh area, Jordan, using } \\
\text { environmental isotope techniques. Hydrogeology Journal, 14, 180-191 (2006). }\end{array}$ \\
\hline 14 & $\begin{array}{l}\text { Bakari, S. S., Aagaard, P., Vogt, R. D., Ruden, F., Johansen, I., Vuai, S. A. Delineation of groundwater provenance in a } \\
\text { coastal aquifer using statistical and isotopic methods, Southeast Tanzania. Environmental Earth Sciences, 66, 889-902 } \\
\text { (2012). }\end{array}$ \\
\hline 15 & $\begin{array}{l}\text { Balderer, Synal, H. A., Deak, J. Application of the chlorine-36 method for the delineation of groundwater infiltration of } \\
\text { large river systems: example of the Danube River in western Hungary (Szigetköz area). Environmental Geology, } 46 \text {, } \\
755-762 \text { (2004). }\end{array}$ \\
\hline 16 & $\begin{array}{l}\text { Berg, J. A., Pearson, S. R. South-central Minnesota groundwater monitoring of the Mt. Simon aquifer - Phase } 2 . \\
\text { Minnesota Department of Natural Resources, Ecological and Water Resources Division report, } 92 \text { pp. (2012). }\end{array}$ \\
\hline 17 & $\begin{array}{l}\text { Beyerle, U., Purtschert, R., Aeschbach-Hertig, W., Imboden, D. M., Loosli, H. H., Wieler, R., Kipfer, R. Climate and } \\
\text { groundwater recharge during the last glaciation in an ice-covered region. Science, 282, 731-734 (1998). }\end{array}$ \\
\hline 18 & $\begin{array}{l}\text { Blomqvist, R. Hydrochemistry of deep groundwaters in the central part of the Fennoscandian Shield. Geological } \\
\text { Survey of Finland Report YST-101, } 41 \text { pp. (1999). }\end{array}$ \\
\hline 19 & $\begin{array}{l}\text { Böhlke, J. K., Révész, K., Busenberg, E., Deák, J., Deseö, E., Stute, M. Groundwater record of halocarbon transport by } \\
\text { the Danube river. Environmental Science \& Technology, 31, 3293-3299 (1998). }\end{array}$ \\
\hline 20 & $\begin{array}{l}\text { Bouchaou, L., Michelot, J. L., Qurtobi, M., Zine, N., Gaye, C. B., Aggarwal, P. K., Marah, H., Zerouali, A., Taleb, H., } \\
\text { Vengosh, A. Origin and residence time of groundwater in the Tadla basin (Morocco) using multiple isotopic and } \\
\text { geochemical tools. Journal of Hydrology, 379, 323-338 (2009). }\end{array}$ \\
\hline 21 & $\begin{array}{l}\text { Bretzler, A., Osenbrück, K., Gloaguen, R., Ruprecht, J. S., Kebede, S., Stadler, S Groundwater origin and flow } \\
\text { dynamics in active rift systems-A multi-isotope approach in the Main Ethiopian Rift. Journal of Hydrology, 402, 274- } \\
289 \text { (2011). }\end{array}$ \\
\hline 22 & $\begin{array}{l}\text { Brown, C. J., Starn, J., Stollenwerk, K. G., Mondazzi, R. A., Trombley, T. J. Aquifer chemistry and transport processes } \\
\text { in the zone of contribution to a public-supply well in Woodbury, Connecticut, 2002-06. US Geological Survey, } \\
\text { Scientific Investigations Report 2009-5051, } 176 \text { pp. (2009). }\end{array}$ \\
\hline 23 & $\begin{array}{l}\text { Busenberg, E., Plummer, L. N. Use of chlorofluorocarbons (CCl3F and CCl2F2) as hydrologic tracers and age-dating } \\
\text { tools: The alluvium and terrace system of central Oklahoma. Water Resources Research, 28, 2257-2283 (1992). }\end{array}$ \\
\hline
\end{tabular}




\begin{tabular}{|c|c|}
\hline \# & \\
\hline 24 & $\begin{array}{l}\text { Calmels, F., Delisle, G., Allard, M. Internal structure and the thermal and hydrological regime of a typical lithalsa: } \\
\text { significance for permafrost growth and decay. Canadian Journal of Earth Sciences, 45, 31-43 (2008). }\end{array}$ \\
\hline 25 & $\begin{array}{l}\text { Carrillo-Rivera, J. J., Clark, I. D., Fritz, P. Investigating recharge of shallow and paleo-groundwaters in the Villa de } \\
\text { Reyes Basin, SLP, Mexico, with environmental isotopes. Applied Hydrogeology, 1, 35-48 (1992). }\end{array}$ \\
\hline 26 & $\begin{array}{l}\text { Cartwright, I., Morgenstern, U. Constraining groundwater recharge and the rate of geochemical processes using tritium } \\
\text { and major ion geochemistry: Ovens catchment, southeast Australia. Journal of Hydrology, 475, 137-149 (2012). }\end{array}$ \\
\hline 27 & $\begin{array}{l}\text { Cartwright, I. Weaver, T., Cendón, D. I., Swane, I. Environmental isotopes as indicators of inter-aquifer mixing, } \\
\text { Wimmera region, Murray Basin, Southeast Australia. Chemical Geology, 277, 214-226 (2010). }\end{array}$ \\
\hline 28 & $\begin{array}{l}\text { Cartwright, I., Weaver, T. R. Hydrogeochemistry of the Goulburn Valley region of the Murray Basin, Australia: } \\
\text { implications for flow paths and resource vulnerability. Hydrogeology Journal, 13, 752-770 (2005). }\end{array}$ \\
\hline 29 & $\begin{array}{l}\text { Cartwright, I., Weaver, T. R., Cendon, D. I., Fifield, L. K., Tweed, S. O., Petrides, B., Swane, I. Constraining } \\
\text { groundwater flow, residence times, inter-aquifer mixing, and aquifer properties using environmental isotopes in the } \\
\text { southeast Murray Basin, Australia. Applied Geochemistry, 27, 1698-1709 (2012). }\end{array}$ \\
\hline 30 & $\begin{array}{l}\text { Cheikh, N. B., Zouari, K., Abidi, B. Geochemical and isotopic study of paleogroundwater salinization in southeastern } \\
\text { Tunisia (Sfax basin). Quaternary International, 257, 34-42 (2012). }\end{array}$ \\
\hline 31 & $\begin{array}{l}\text { Chen, L., Wang, G., Hu, F., Wang, Y., Liu, L. Groundwater hydrochemistry and isotope geochemistry in the Turpan } \\
\text { Basin, northwestern China. Journal of Arid Land, 6, 378-388 (2014). }\end{array}$ \\
\hline 32 & $\begin{array}{l}\text { Choi, B-Y., Yun, S-T., Yu, S-Y., Lee, P-K., Park, S-S., Chae, G-T., Mayer, B. Hydrochemistry of urban groundwater } \\
\text { in Seoul, South Korea: effects of land-use and pollutant recharge. Environmental Geology, 48, 979-990 (2005). }\end{array}$ \\
\hline 33 & $\begin{array}{l}\text { Clark, I. D., Fritz, P., Quinn, O. P., Rippon, P. W., Nash, H., al Said, S. B. B. G. Modern and fossil groundwater in an } \\
\text { arid environment: a look at the hydrogeology of southern Oman. in: Isotope Techniques in Water Resources } \\
\text { Development, International Atomic Energy Agency, Vienna, Austria, 167-187 (1987). }\end{array}$ \\
\hline 34 & $\begin{array}{l}\text { Cook, P. G., Love, A. J., Robinson, N. I., Simmons, C. T. Groundwater ages in fractured rock aquifers. Journal of } \\
\text { Hydrology, 308, 284-301 (2005). }\end{array}$ \\
\hline 35 & $\begin{array}{l}\text { Cook, P. G., Solomon, D. K., Plummer, L. N., Busenberg, E., Schiff, S. L. Chlorofluorocarbons as tracers of } \\
\text { groundwater transport processes in a shallow, silty sand aquifer. Water Resources Research, 31, 425-434 (1995). }\end{array}$ \\
\hline 36 & $\begin{array}{l}\text { Corcho Alvarado, J. A., Pačes, T., Purtschert, R. Dating groundwater in the Bohemian Cretaceous Basin: } \\
\text { Understanding tracer variations in the subsurface. Applied Geochemistry, 29, 189-198 (2011). }\end{array}$ \\
\hline 37 & $\begin{array}{l}\text { Cortecci, G., Dinelli, E., Bolognesi, L., Boschetti, T., Ferrara, G. Chemical and isotopic compositions of water and } \\
\text { dissolved sulfate from shallow wells on Vulcano Island, Aeolian Archipelago, Italy. Geothermics, 30, 69-91 (2001). }\end{array}$ \\
\hline 38 & $\begin{array}{l}\text { Cronin, A. A., Barth, J. A. C., Elliot, T., Kalin, R. M. Recharge velocity and geochemical evolution for the Permo- } \\
\text { Triassic Sherwood Sandstone, Northern Ireland. Journal of Hydrology, 315, 308-324 (2005). }\end{array}$ \\
\hline 39 & $\begin{array}{l}\text { Currell, M. J., Cartwright, I., Bradley, D. C., Han, D. Recharge history and controls on groundwater quality in the } \\
\text { Yuncheng Basin, north China. Journal of Hydrology, 385, 216-229 (2010). }\end{array}$ \\
\hline 40 & $\begin{array}{l}\text { Dakin, R. A.m Farvolden, R. N., Cherry, J. A., Fritz, P. Origin of dissolved solids in groundwaters of Mayne Island, } \\
\text { British Columbia, Canada. Journal of Hydrology, 63, 233-270 (1983). }\end{array}$ \\
\hline 41 & $\begin{array}{l}\text { Demlie, M., Wohnlich, S., Gizaw, B., Stichler, W. Groundwater recharge in the Akaki catchment, central Ethiopia: } \\
\text { evidence from environmental isotopes ( } 1 \text { 18O, d2H and 3H) and chloride mass balance. Hydrological Processes, } 21 \text {, } \\
807-818 \text { (2007). }\end{array}$ \\
\hline 42 & $\begin{array}{l}\text { Derwich, L. J., Zouar, K., Michelot, J. L. Recharge and paleorecharge of the deep groundwater aquifer system in the } \\
\text { Zeroud Basin (Kairouan plain, Central Tunisia). Quaternary International, 257, 56-63 (2012). }\end{array}$ \\
\hline 43 & $\begin{array}{l}\text { Desaulniers, D. E., Cherry, J. A., Fritz, P. Origin, age and movement of pore water in argillaceous Quaternary deposits } \\
\text { at four sites in southwestern Ontario. Journal of Hydrology, 50, 231-257 (1981). }\end{array}$ \\
\hline 44 & $\begin{array}{l}\text { Dupalová, T., Sracek, O., Vencelides, Z., Zák, K. The origin of thermal waters in the northeastern part of the Eger Rift, } \\
\text { Czech Republic. Applied Geochemistry, 27, 689-702 (2012). }\end{array}$ \\
\hline 45 & $\begin{array}{l}\text { Eastoe, C. J., Rodney, R. Isotopes as Tracers of Water Origin in and Near a Regional Carbonate Aquifer: The Southern } \\
\text { Sacramento Mountains, New Mexico. Water, 6, 301-323 (2014). }\end{array}$ \\
\hline 46 & $\begin{array}{l}\text { Edmunds, W. M., Carrillo-Rivera, J. J., Cardona, A. Geochemical evolution of groundwater beneath Mexico City. } \\
\text { Journal of Hydrology, 258, 1-24 (2002). }\end{array}$ \\
\hline 47 & $\begin{array}{l}\text { Edmunds, W. M., Wright, E. P. Groundwater recharge and paleoclimate in the Sirte and Kufra basins, Libya. Journal of } \\
\text { Hydrology, 40, 215-241 (1979). }\end{array}$ \\
\hline 48 & $\begin{array}{l}\text { Fass, T., Cook, P. G., Stieglitz, T., Herczeg, A. L. Development of saline ground water through transpiration of sea } \\
\text { water. Groundwater, } 45,703-710 \text { (2007). }\end{array}$ \\
\hline 49 & $\begin{array}{l}\text { Fontes, J.-C., Andrews, J. N., Edmunds, W. M., Guerre, A., Travi, Y. Paleorecharge by the Niger river (Mali) deduced } \\
\text { from groundwater geochemistry. Water Resources Research, 27, 199-214 (1991). }\end{array}$ \\
\hline 50 & $\begin{array}{l}\text { Foriz, I., Berecz, T., Molnar, Z., Suveges, M. Origin of shallow groundwater of Csepel Island (south of Budapest, } \\
\text { Hungary, River Danube): isotopic and chemical approach. Hydrological Processes, 19, 3299-3312 (2005). }\end{array}$ \\
\hline 51 & $\begin{array}{l}\text { Friedrich, R., Vero, G., von Rohden, C., Lessmann, B., Kipfer, R., \& Aeschbach-Hertig, W. Factors controlling } \\
\text { terrigenic SF } 6 \text { in young groundwater of the Odenwald region (Germany). . Applied Geochemistry, 33, 318-329 (2013) }\end{array}$ \\
\hline
\end{tabular}




\begin{tabular}{|c|c|}
\hline$\#$ & eference \\
\hline 52 & $\begin{array}{l}\text { Geirnaert, W., Laeven, M. P. Composition and history of ground water in the western Nile Delta. Journal of Hydrology, } \\
138,169-189 \text { (1992). }\end{array}$ \\
\hline 53 & $\begin{array}{l}\text { Gerber, R. E., Howard, K. W. F. Evidence for recent groundwater flow through Late Wisconsinan till near Toronto, } \\
\text { Ontario. Canadian Geotechnical Journal, 33, 538-555 (2001). }\end{array}$ \\
\hline 54 & $\begin{array}{l}\text { Geyh, M. A., Ploethner, D. Origin of a fresh groundwater body in Cholistan, Thar Desert, Pakistan. Geological Society, } \\
\text { London, Special Publications, 288, 99-109 (2008). }\end{array}$ \\
\hline 55 & $\begin{array}{l}\text { Gonfiantini, R., Simonot, M. Isotopic investigation of groundwater in the Cul-de-sac Plain, Haiti. in: Isotope techniques } \\
\text { in water resources development, International Atomic Energy Agency, Vienna, Austria, 483-504 (1987). }\end{array}$ \\
\hline 56 & $\begin{array}{l}\text { Grassi, S., Cortecci, G. Hydrogeology and geochemistry of the multilayered confined aquifer of the Pisa plain } \\
\text { (Tuscany-central Italy). Applied Geochemistry, 20, 41-54 (2005). }\end{array}$ \\
\hline 57 & $\begin{array}{l}\text { Harrington, G., Stelfox, L., Gardner, P., Davies, P., Doble, R., Cook, P. Surface Water - Groundwater Interactions in } \\
\text { the Lower Fitzroy River, Western Australia. CSIRO report: Water for a Healthy Country National Research Flagship, } \\
54 \text { pp. (2011). }\end{array}$ \\
\hline 58 & $\begin{array}{l}\text { Hatipoglu, Z., Motz, L. H., Bayari, C. S. Characterization of the groundwater flow system in the hillside and coastal } \\
\text { aquifers of the Mersin-Tarsus region (Turkey). Hydrogeology Journal, 17, 1761-1778 (2009). }\end{array}$ \\
\hline 59 & $\begin{array}{l}\text { He, J., Ma, J., Zhang, P., Tian, L., Zhu, G., Edmunds, W. M., Zhang, Q. Groundwater recharge environments and } \\
\text { hydrogeochemical evolution in the Jiuquan Basin, Northwest China. Applied Geochemistry, 27, 866-878 (2012). }\end{array}$ \\
\hline 60 & $\begin{array}{l}\text { Horst, A. Use of Stable and Radioactive Isotopes and Gase-ous Tracers for Estimating Groundwater Recharge, Time of } \\
\text { Residence, Mixing of the Different Types of Groundwater and Origin in the Silao Romita Aquifer, Guanajuato, Central } \\
\text { Mexico. Freiberg Online Journal, 17, } 119 \text { pp. (2006). }\end{array}$ \\
\hline 61 & $\begin{array}{l}\text { Houben, G. J., Koeniger, P., Sultenfuß, J. Freshwater lenses as archive of climate, groundwater recharge, and } \\
\text { hydrochemical evolution: Insights from depth-specific water isotope analysis and age determination on the island of } \\
\text { Langeoog, Germany. Water Resources Research, 50, 8227-8239 (2014). }\end{array}$ \\
\hline 62 & $\begin{array}{l}\text { Houston, J. Recharge to groundwater in the Turi Basin, northern Chile: an evaluation based on tritium and chloride } \\
\text { mass balance techniques. Journal of hydrology, 334, 534-544 (2007). }\end{array}$ \\
\hline 63 & $\begin{array}{l}\text { Huang, T., Pang, Z. Changes in groundwater induced by water diversion in the Lower Tarim River, Xinjiang Uygur, } \\
\text { NW China: Evidence from environmental isotopes and water chemistry. Journal of Hydrology, 387, 188-201 (2010). }\end{array}$ \\
\hline 64 & $\begin{array}{l}\text { Huff, G. F., Woods, L., Moktan, H., Jean, G. Geochemistry of groundwater and springwater in the Paskapoo formation } \\
\text { and overlying glacial drift, south-central Alberta. ERCB/AGS Open File Report 2012-05, } 66 \text { pp. (2012). }\end{array}$ \\
\hline 65 & $\begin{array}{l}\text { Ingraham, N. L., Matthews, R. A. Fog drip as a source of groundwater recharge in northern Kenya. Water Resources } \\
\text { Research, 24, 1406-1410 (1988). }\end{array}$ \\
\hline 66 & $\begin{array}{l}\text { Iriarte, S., Atenas, M., Aguirre, E., Tore, C. Aquifer recharge and contamination determination using environmental } \\
\text { isotopes: Santiago basin, Chile A study case. in: Estudios de Hidrología Isotópica en América Latina, IAEA-TECDOC- } \\
\text { 1611, 97-112 (2006). }\end{array}$ \\
\hline 67 & $\begin{array}{l}\text { Iwatsuki, T., Xu, S., Itoh, S., Abe, M., Watanabe, M. Estimation of relative groundwater age in the granite at the Tono } \\
\text { research site, central Japan. Nuclear Instruments and Methods in Physics Research Section B: Beam Interactions with } \\
\text { Materials and Atoms, 172, 524-529 (2000). }\end{array}$ \\
\hline 68 & $\begin{array}{l}\text { Jiráková, H., Huneau, F., Hrkal, Z., Celle-Jeanton, H., Le Coustumer, P. Carbon isotopes to constrain the origin and } \\
\text { circulation pattern of groundwater in the north-western part of the Bohemian Cretaceous Basin (Czech Republic). } \\
\text { Applied Geochemistry, 25, 1265-1279 (2010). }\end{array}$ \\
\hline 69 & $\begin{array}{l}\text { Kamdee, K., Srisuk, K., Lorphensri, O., Chitradon, R., Noipow, N., Laoharojanaphand, S., Chantarachot, W. Use of } \\
\text { isotope hydrology for groundwater resources study in Upper Chi river basin. Journal of Radioanalytical and Nuclear } \\
\text { Chemistry, 297, 405-418 (2013). }\end{array}$ \\
\hline 70 & $\begin{array}{l}\text { Kaown, D., Koh, D-C., Lee, K-L. Effects of groundwater residence time and recharge rate on nitrate contamination } \\
\text { deduced from d18O, dD, 3H/3He and CFCs in a small agricultural area in Chuncheon, Korea. Journal of Hydrology, } \\
\text { 366, 101-111 (2009). }\end{array}$ \\
\hline 71 & $\begin{array}{l}\text { Kattan, Z. Characterization of surface water and groundwater in the Damascus Ghotta basin: hydrochemical and } \\
\text { environmental isotopes approaches. Environmental Geology, 51, 173-201 (2006). }\end{array}$ \\
\hline 72 & $\begin{array}{l}\text { Kattan, Z. Use of hydrochemistry and environmental isotopes for evaluation of groundwater in the Paleogene limestone } \\
\text { aquifer of the Ras Al-Ain area (Syrian Jezireh). Environmental Geology, 41, 128-144 (2001). }\end{array}$ \\
\hline 73 & $\begin{array}{l}\text { Kebede, S., Travi, Y., Asrat, A., Alemayehu, T., Ayenew, T., Tessema, Z. Groundwater origin and flow along selected } \\
\text { transects in Ethiopian rift volcanic aquifers. Hydrogeology Journal, 16, 55-73 (2007). }\end{array}$ \\
\hline 74 & $\begin{array}{l}\text { King, A. C., Raiber, M., Cendon, D. I., Cox, M. E., Hollins, S. E. Identifying flood recharge and inter-aquifer } \\
\text { connectivity using multiple isotopes in subtropical Australia. Hydrology and Earth System Sciences, 11, 3711-3756 } \\
\text { (2014). }\end{array}$ \\
\hline 75 & $\begin{array}{l}\text { Külls, C. Groundwater of the North-Western Kalahari, Namibia: Estimation of recharge and quantification of the flow } \\
\text { systems. PhD dissertation, University of Würzburg, } 230 \mathrm{pp}(2000) \text {. }\end{array}$ \\
\hline 76 & $\begin{array}{l}\text { Kulongoski, J. T., Hilton, D. R., Cresswell, R. G., Hostetler, S., Jacobsen, G. Helium-4 characteristics of groundwaters } \\
\text { from Central Australia: Comparative chronology with chlorine-36 and carbon-14 dating techniques. Journal of } \\
\text { Hydrology, 348, 176-194 (2008). }\end{array}$ \\
\hline
\end{tabular}




\begin{tabular}{|c|c|}
\hline \# & ference \\
\hline 77 & $\begin{array}{l}\text { Kumar, B., Rao, M. S., Gupta, A. K., Purushothaman, P. Groundwater management in a coastal aquifer in Krishna } \\
\text { River Delata, South India using an isotopic approach. Current Science, 100, 1032-1043 (2011). }\end{array}$ \\
\hline 78 & $\begin{array}{l}\text { Kusano, Y., Tokunaga, T., Asai, K., Takahashi, H. A., Morikawa, N., Yasuhara, M. Occurrence of old groundwater in a } \\
\text { volcanic island on a continental shelf; an example from Nakano-shima Island, Oki-Dozen, Japan. Journal of Hydrology, } \\
511,295-309 \text { (2014). }\end{array}$ \\
\hline 79 & $\begin{array}{l}\text { Lavastre, V., Le Gal La Salle, C., Michelot, J-L., Giannesinia, S., Benedettia, L., Lancelota, J., Lavielle, B., Massault, } \\
\text { M., Thomas, B., Gilabert, E., Bourlès, D., Clauer, N., Agrinier, P. Establishing constraints on groundwater ages with } \\
{ }^{36} \mathrm{Cl},{ }^{14} \mathrm{C},{ }^{3} \mathrm{H} \text {, and noble gases: A case study in the eastern Paris basin, France. Applied Geochemistry, 25, 123-142 } \\
(2010) \text {. }\end{array}$ \\
\hline 80 & $\begin{array}{l}\text { Le Gal La Salle, C., Fontes, J-C., Andrews, J. N., Schroeter, P., Karbo, A., Fifield, K. L. Old groundwater circulation in } \\
\text { the Illumeden basin (Niger): preliminary results of an isotopic study. International Atomic Energy Agency Conference: } \\
\text { Applications of Tracers in Arid Zone Hydrology, Vienna, 129-139 (1995). }\end{array}$ \\
\hline 81 & $\begin{array}{l}\text { Le Gal La Salle, C., Marlin, C., Savoye, S., Fontes, J-F. Geochemistry and 14C dating of groundwaters from Jurassic } \\
\text { aquifers of North Aquitaine Basin (France). Applied Geochemistry, 11, 433-445 (1995). }\end{array}$ \\
\hline 82 & $\begin{array}{l}\text { Leaney, F. W., Allison, G. B. Carbon-14 and stable isotope data for an area in the Murray basin: its use in estimating } \\
\text { recharge. Journal of Hydrology, 88, 129-145 (1986). }\end{array}$ \\
\hline 83 & $\begin{array}{l}\text { Lehmann, B. E., Love, A., Purtschert, R., Collon, P., Loosli, H. H., Kutschera, W., Beyerle, U., Aeschbach-Hertig, W., } \\
\text { Kipfer, R., Frape, S. K., Herczeg, A., Moran, J., Tolstikhin, I. N., Gröening, M. A comparison of groundwater dating } \\
\text { with }{ }^{81} \mathrm{Kr},{ }^{36} \mathrm{Cl} \text { and }{ }^{4} \mathrm{He} \text { in four wells of the Great Artesian Basin, Australia. Earth and Planetary Science Letters, } 211 \text {, } \\
237-250(2003) \text {. }\end{array}$ \\
\hline 84 & $\begin{array}{l}\text { Leontiadis, I. L., Vergis, S., Christodoulou, T. Isotope hydrology study of areas in Eastern Macedonia and Thrace, } \\
\text { Northern Greece. Journal of Hydrology, 182, 1-17 (1996). }\end{array}$ \\
\hline 85 & $\begin{array}{l}\text { Li, J., Pang, Z., Froehlich, K., Huang, T., Kong, Y., Song, W., Yun, H. Paleo-environment from isotopes and } \\
\text { hydrochemistry of groundwater in East Junggar Basin, Northwest China. Journal of Hydrology, 529, 650-661 (2015). }\end{array}$ \\
\hline 86 & $\begin{array}{l}\left.\text { Liu, J., Chen, Z., Wei, W., Zhang, Y., Li, Z., Liu, F., Guo, H Using chlorofluorocarbons (CFCs) and tritium ( }{ }^{3} \mathrm{H}\right) \text { to } \\
\text { estimate groundwater age and flow velocity in Hohhot Basin, China. Hydrological Processes, 28, 1372-1382 (2014). }\end{array}$ \\
\hline 87 & $\begin{array}{l}\text { Ma, J., Ding, Z., Edmunds, W. M., Gates, J. B., Huang, T. Limits to recharge of groundwater from Tibetan plateau to } \\
\text { the Gobi desert, implications for water management in the mountain front. Journal of Hydrology, 364, 128-141 (2009). }\end{array}$ \\
\hline 88 & $\begin{array}{l}\text { Ma, J., He, J., Qi, S., Zhu, G., Zhao, W., Edmunds, W. M., Zhao, Y. Groundwater recharge and evolution in the } \\
\text { Dunhuang Basin, northwestern China. Applied Geochemistry, 28, 19-31 (2013). }\end{array}$ \\
\hline 89 & $\begin{array}{l}\text { Ma, L., Castro, M. C., Hall, C. M. A late Pleistocene-Holocene noble gas paleotemperature record in southern } \\
\text { Michigan. Geophysical Research Letters, 31, L23204 (2004). }\end{array}$ \\
\hline 90 & $\begin{array}{l}\text { Mack, T. J., Chornack, M. P., Flanagan, S. M., Chalmers, A. T. Hydrogeology and Water Quality of the Chakari Basin, } \\
\text { Afghanistan. Scientific Investigations Report 2014-5113, } 33 \text { pp. (2014). }\end{array}$ \\
\hline 91 & $\begin{array}{l}\text { Mahara, Y., Igarashi, T. Changes in isotope ratio and content of dissolved helium through groundwater evolution. } \\
\text { Applied Geochemistry, 18, 719-738 (2003). }\end{array}$ \\
\hline 92 & $\begin{array}{l}\text { Mahara, Y., Ohta, T., Morikawa, N., Nakano, T., Tokumasu, M., Hukutani, S., Tokunaga, T., Igarashi, T. Effects of } \\
\text { terrigenic He components on tritium-helium dating: A case study of shallow groundwater in the Saijo Basin. Applied } \\
\text { Geochemistry, 50,142-149 (2014). }\end{array}$ \\
\hline 93 & $\begin{array}{l}\text { Mahlknecht, J., Gárfias-Solis, J., Aravena, R., Tesch, R. Geochemical and isotopic investigations on } \\
\text { residence time and flow in the Independence Basin, Mexico. Journal of Hydrology, 324, 283-300 (20 }\end{array}$ \\
\hline 94 & $\begin{array}{l}\text { Mahlknecht, J., Medina-Mejía, M. G., Gárfias-Solis, J., Cano-Aguilera, I. Intrinsic aquifer vulnerability assessment: } \\
\text { validation by environmental tracers in San Miguel de Allende, Mexico. Environmental Geology, 51, 477-491 (2006). }\end{array}$ \\
\hline 95 & $\begin{array}{l}\text { Maldonado Astudillo, S., Cepeda Tobar, H., Araguás-Araguás, L. Estudio hldrogeologico e isotópico de las aguas } \\
\text { subterráneas del área de Chacras Huaquillas (Ecuador). in: Estudios de hidrología isotópica en América Latina, IAEA- } \\
\text { TECDOC-835, 211-236 (1994). }\end{array}$ \\
\hline 96 & $\begin{array}{l}\text { Manning, A. H., Caine, J. S. Groundwater noble gas, age, and temperature signatures in an Alpine watershed: Valuable } \\
\text { tools in conceptual model development. Water Resources Research, 43, W04404 (2007). }\end{array}$ \\
\hline 97 & $\begin{array}{l}\text { Matter, J. M., Waber, H. N., Loew, S., Matter, A. Recharge areas and geochemical evolution of groundwater in an } \\
\text { alluvial aquifer system in the Sultanate of Oman. Hydrogeology Journal, 14, 203-224 (2005). }\end{array}$ \\
\hline 98 & $\begin{array}{l}\text { Mažeika, J., Martma, T., Petrošius, R., JakimaviËiūtė-Maselienė, V., Skuratovič, Z. Radiocarbon and other } \\
\text { environmental isotopes in the groundwater of the sites for a planned new nuclear power plant in Lithuania. } \\
\text { Radiocarbon, 55, 951-962 (2013). }\end{array}$ \\
\hline 99 & $\begin{array}{l}\text { Meredith, K., Cendón, D. I., Pigois, J-P., Hollins, S., Jacobsen, G. Using } 14 \mathrm{C} \text { and } 3 \mathrm{H} \text { to delineate a recharge 'window' } \\
\text { into the Perth Basin aquifers, North Gnangara groundwater system, Western Australia. Science of the Total } \\
\text { Environment, 414, 456-469 (2012). }\end{array}$ \\
\hline 100 & $\begin{array}{l}\text { Michel, F. A., Allen, D. M., Grant, M. B. Hydrogeochemistry and geothermal characteristics of the White Lake basin, } \\
\text { South-central British Columbia, Canada. Geothermics, 31, 169-194 (2002). }\end{array}$ \\
\hline 101 & $\begin{array}{l}\text { Mokrik, R., Juodkazis, V., Štuopis, A., Mažeika, J. Isotope geochemistry and modelling of the multi-aquifer system in } \\
\text { the eastern part of Lithuania. Hydrogeology Journal, 22, 925-941 (2014). }\end{array}$ \\
\hline
\end{tabular}




\begin{tabular}{|c|c|}
\hline \# & \\
\hline 102 & $\begin{array}{l}\text { Mokrik, R., Mažeika, J., Baublytè, A., Martma, T. The groundwater age in the Middle-Upper Devonian aquifer system, } \\
\text { Lithuania. Hydrogeology Journal, 17, 871-889 (2009). }\end{array}$ \\
\hline 103 & $\begin{array}{l}\text { Moussa, A. B., Salem, S. B. H., Zouari, K., Jlassi, F. Hydrochemical and isotopic investigation of the groundwater } \\
\text { composition of an alluvial aquifer, Cap Bon Peninsula, Tunisia. Carbonates Evaporites, 25, 161-176 (2010). }\end{array}$ \\
\hline 104 & $\begin{array}{l}\text { Murphy, S., Ouellon, T., Ballard, J. M., Lefebvre, R., Clark, I. D. Tritium-helium groundwater age used to constrain a } \\
\text { groundwater flow model of a valley-fill aquifer contaminated with trichloroethylene (Quebec, Canada). Hydrogeology } \\
\text { Journal, 19, 195-207 (2010). }\end{array}$ \\
\hline 105 & $\begin{array}{l}\text { Navada, S. V., Nair, A. R., Rao, S. M., Paliwall, B. L., Doshi, C. S. Groundwater recharge studies in arid region of } \\
\text { Jalore, Rajasthan using isotope techniques. Journal of Arid Environments, 24, 125-133 (1993). }\end{array}$ \\
\hline 106 & $\begin{array}{l}\text { Njitchoua, R., Fontes, J-Ch., Zuppi, G. M. Use of chemical and isotopic tracers in studying the recharge processes of } \\
\text { the upper Cretaceous aquifer of the Garoua basin, northern Cameroon. in: Application of Tracers in Arid Zone } \\
\text { Hydrology, International Atomic Energy Agency Conference Proceedings, 232, 363-372 (1995). }\end{array}$ \\
\hline 107 & $\begin{array}{l}\text { Noseck, U., Rozanski, K., Dulinski, M., Havlová, V., Sracek, O., Brasser, T., Hercik, M., Buckau, G. Carbon chemistry } \\
\text { and groundwater dynamics at natural analogue site Ruprechtov, Czech Republic: Insights from environmental isotopes. } \\
\text { Applied Geochemistry, 24, 1765-1776 (2009). }\end{array}$ \\
\hline 108 & $\begin{array}{l}\text { Nurmi, P. A., Kukkonen, I. T., Lahermo, P. W. Geochemistry and origin of saline groundwaters in the Fennoscandian } \\
\text { Shield. Applied Geochemistry, 3, 185-203 (1988). }\end{array}$ \\
\hline 109 & $\begin{array}{l}\text { Pang, Z., Yuan, L., Huang, T., Kong, Y., Liu, J., Li, Y. Impacts of human activities on the occurrence of groundwater } \\
\text { nitrate in an alluvial plain: a multiple isotopic tracers approach. Journal of Earth Science, 24, 111-124 (2013). }\end{array}$ \\
\hline 110 & $\begin{array}{l}\text { Payne, B. R., Yurtsever, Y. Environmental isotopes as a hydrogeological tool in Nicaragua. in: Isotope techniques in } \\
\text { groundwater hydrology 1974, Vol. I., International Atomic Energy Agency, Vienna, Austria, 193-200 (1974). }\end{array}$ \\
\hline 111 & $\begin{array}{l}\text { Peng, T. R., Huang, C. C., Wang, C. H., Liu, T. K., Lu, W. C., Chen, K. Y. Using oxygen, hydrogen, and tritium } \\
\text { isotopes to assess pond water's contribution to groundwater and local precipitation in the pediment tableland areas of } \\
\text { northwestern Taiwan. Journal of Hydrology, 450, 105-116 (2012). }\end{array}$ \\
\hline 112 & $\begin{array}{l}\text { Perrin, J., Parker, B. L., Cherry, J. A. Assessing the flow regime in a contaminated fractured and karstic dolostone } \\
\text { aquifer supplying municipal water. Journal of Hydrology, 400, 396-410 (2011). }\end{array}$ \\
\hline 113 & $\begin{array}{l}\text { Pilla, G., Sacchi, E., Zuppi, G., Braga, G., Ciancetti, G. Hydrochemistry and isotope geochemistry as tools for } \\
\text { groundwater hydrodynamic investigation in multilayer aquifers: a case study from Lomellina, Po plain, South-Western } \\
\text { Lombardy, Italy. Hydrochemistry and isotope geochemistry as tools for groundwater hydrodynamic investigation in } \\
\text { multilayer aquifers: a case study from Lomellina, Po plain, South-Western Lombardy, Italy, 14, 795-808 (2006). }\end{array}$ \\
\hline 114 & $\begin{array}{l}\text { Plummer, L. N., Bexfield, L. M., Anderholm, S. K., Sanford, W. E., Busenberg, E. Hydrochemical tracers in the middle } \\
\text { Rio Grande Basin, USA: 1. Conceptualization of groundwater flow. Hydrogeology Journal, 12, 359-388 (2004). }\end{array}$ \\
\hline 115 & $\begin{array}{l}\text { Plummer, L. N., Rupert, M. G., Busenberg, E., Schlosser, P. Age of Irrigation Water in Ground Water from the Eastern } \\
\text { Snake River Plain Aquifer, South-Central Idaho. Groundwater, 38, 264-283 (2000). }\end{array}$ \\
\hline 116 & $\begin{array}{l}\text { Povinec, P. P., Zenisova, Z., Breier, R., Richtarikova, M., Sivo, A. Isotopic tracing of groundwater at Zitny ostrov (SW } \\
\text { Slovakia). EPJ Web of Conferences, 24, } 03004 \text { p1-11 (2012). }\end{array}$ \\
\hline 117 & $\begin{array}{l}\text { Qin, D., Qian, Y., Han, L., Wang, Z., Li, C., Zhao, Z. Assessing impact of irrigation water on groundwater recharge } \\
\text { and quality in arid environment using CFCs, tritium and stable isotopes, in the Zhangye Basin, Northwest China. } \\
\text { Journal of Hydrology, 405, 194-208 (2011). }\end{array}$ \\
\hline 118 & $\begin{array}{l}\text { Qin, D., Zhao, Z., Han, L., Qian, Y., Ou, L., Wu, Z., Wang, M. Determination of groundwater recharge regime and } \\
\text { flowpath in the Lower Heihe River basin in an arid area of Northwest China by using environmental tracers: } \\
\text { Implications for vegetation degradation in the Ejina Oasis. Applied Geochemistry, 27, 1133-1145 (2012). }\end{array}$ \\
\hline 119 & $\begin{array}{l}\text { Rachid, E. M., Bahir, M., Chamchati, H., Najiba, C., Carreira, P. M., Naserrddin, Y. Using geochemical and isotope } \\
\text { investigations for groundwater management strategies under semi-arid area: case of the Wadi Ouazzi Basin (Morocco). } \\
\text { Journal of Environmental and Earth Science, 4, 18-31 (2014). }\end{array}$ \\
\hline 120 & $\begin{array}{l}\text { Raco, B., Dotsika, E., Battaglini, R., Bulleri, E., Doveri, M., Papakostantinou, K. A quick and reliable method to detect } \\
\text { and quantify contamination from MSW landfills: a case study. Water, Air, \& Soil Pollution, 224, 1-18 (2013). }\end{array}$ \\
\hline 121 & $\begin{array}{l}\text { Rauert, W., Wolf, M., Weise, S. M., Andres, G., Egger, R. Isotope-hydrogeological case study on the penetration of } \\
\text { pollution into the deep Tertiary aquifer in the area of Munich, Germany. Journal of Contaminant Hydrology, 14, 15-38 } \\
\text { (1993). }\end{array}$ \\
\hline 122 & $\begin{array}{l}\text { Reddy, D. V., Nagabhushanam, P., Rao, M. R., Mitra, D. S., Tripati, B. M., Sinha, S. K., Bhadu, B. Radiocarbon } \\
\text { evidence of paleo-recharge (pre-Saraswati period) of potential deep aquifer in Thar desert. Journal of the Geological } \\
\text { Society of India, 77, 239-242 (2011). }\end{array}$ \\
\hline 123 & $\begin{array}{l}\text { Saha, D., Sinha, U. K., Dwivedi, S. N. Characterization of recharge processes in shallow and deeper aquifers using } \\
\text { isotopic signatures and geochemical behavior of groundwater in an arsenic-enriched part of the Ganga Plain. Applied } \\
\text { Geochemistry, 26, 432-443 (2011). }\end{array}$ \\
\hline 124 & $\begin{array}{l}\text { Salati, E., Menezes Leal, J., and Mendes Campos, M. Environmental isotopes used in a hydrogeological study of } \\
\text { northeastern Brazil. Isotope Techniques in Groundwater Hydrology, Vol. I., 379-398 (1974). }\end{array}$ \\
\hline 125 & $\begin{array}{l}\text { Santiago, M. F., Silva, C. M. S. V., Mendes Filho, J., Frischkorn, H. Characterization of groundwater in the Cariri } \\
\text { (Ceara, Brazil) by environmental isotopes and electric conductivity. Radiocarbon, 39, 49-59 (1997). }\end{array}$ \\
\hline
\end{tabular}




\begin{tabular}{|c|c|}
\hline \# & Reference \\
\hline 126 & $\begin{array}{l}\text { Satrio, Paston, S., Chung Sum, L., Syafalni, S. Groundwater Dynamic and Its Interrelationship with River Water of } \\
\text { Bandung Basin Using Environmental Isotopes }\left({ }^{18} \mathrm{O},{ }^{2} \mathrm{H},{ }^{14} \mathrm{C}\right) \text {. Modern Applied Science, 6, 49-59 (2012). }\end{array}$ \\
\hline 127 & $\begin{array}{l}\text { Schlosser, P., Stute, M., Sonntag, C., Munnich, K. O. Tritiogenic 3He in shallow groundwater. Earth and Planetary } \\
\text { Science Letters, 94, 245-256 (1988). }\end{array}$ \\
\hline 128 & Seletsky, Y. B. Isotope studies of groundwater in South Kazakhstan. Soviet Geology, 2, 112 (1983). \\
\hline 129 & $\begin{array}{l}\text { Shapiro, A. M. Effective matrix diffusion in kilometer-scale transport in fractured crystalline rock. Water Resources } \\
\text { Research, 37, 507-522 (2001). }\end{array}$ \\
\hline 130 & $\begin{array}{l}\text { Shehata, M., Al-Ruwaih, F. Major ions geochemistry and environmental isotope study of the Nubian Aquifer system, } \\
\text { Dakhla Oasis, Western Desert, Egypt. Arabian Journal for Science and Engineering, 24, 43-58 (1999). }\end{array}$ \\
\hline 131 & $\begin{array}{l}\text { Shivanna, K., Kulkarni, U. P., Joseph, T. B., \& Navada, S. V. Contribution of storms to groundwater recharge in the } \\
\text { semi-arid region of Karnataka, India. Hydrological Processes, 18, 473-485 (2004). }\end{array}$ \\
\hline 132 & $\begin{array}{l}\text { Silva, A. A. K. E., Reboucas, A. D. C., Santiago, M. M. F. 14C analyses of groundwater from the Botacatu aquifer } \\
\text { system in Brazil. Radiocarbon, 31, 926-933 (1989). }\end{array}$ \\
\hline 133 & $\begin{array}{l}\text { Smellie, J. A. T., Wikberg, P. Hydrochemical investigations at Finnsjön, Sweden. Journal of Hydrology, 126, 129-158 } \\
\text { (1991). }\end{array}$ \\
\hline 134 & $\begin{array}{l}\text { Sokolovskii, L. G., Polyakov, V. A., \& Markidanova, H. S. Isotope composition of groundwater in the Azov-Kuban } \\
\text { and Eastern Pre-Caucasian artesian basins. Water Resources, 39, 322-329 (2012). }\end{array}$ \\
\hline 135 & $\begin{array}{l}\text { Soro, N., Goula Bi Tie, A. Hydrochimie et isotopes de l'environnement appliqués à l'étude des eaux souterraines du Sud } \\
\text { de la Côte d'Ivoire. Hydrochemistry, 244, } 1997 \text { (1997). }\end{array}$ \\
\hline 136 & $\begin{array}{l}\text { Stadler, S., Geyh, M. A., Ploethner, D., Koeniger, P. The deep Cretaceous aquifer in the Aleppo and Steppe basins of } \\
\text { Syria: assessment of the meteoric origin and geographic source of the groundwater. Hydrogeology Journal, 20, 1007- } \\
1026 \text { (2012). }\end{array}$ \\
\hline 137 & $\begin{array}{l}\text { Stewart, M. K. A 40-year record of carbon-14 and tritium in the Christchurch groundwater system, New Zealand: } \\
\text { Dating of young samples with carbon-14. Journal of Hydrology, 430-431, 50-68 (2012). }\end{array}$ \\
\hline 138 & $\begin{array}{l}\text { Stimson, J., Frape, S., Drimmie, R., Rudolph, D. Isotopic and geochemical evidence of regional-scale anisotropy and } \\
\text { interconnectivity of an alluvial fan system, Cochabamba Valley, Bolivia. Applied Geochemistry, 16, 1097-1114 (2001). }\end{array}$ \\
\hline 139 & $\begin{array}{l}\text { Stimson, J.,Rudolph, D., Frape, S., Drimmie, R. Interpretation of groundwater flow patterns through a reconstruction of } \\
\text { the tritium precipitation record in the Cochabamba Valley, Bolivia. Journal of Hydrology, 180, 155-172 (1996). }\end{array}$ \\
\hline 140 & $\begin{array}{l}\text { Stute, M., Clark, J. F., Schlosser, P., Broecker, W. S., Bonani, G. A 30,000 yr continental paleotemperature record } \\
\text { derived from noble gases dissolved in groundwater from the San Juan Basin, New Mexico. Quaternary Research, 43, } \\
\text { 209-220 (1995). }\end{array}$ \\
\hline 141 & $\begin{array}{l}\text { Sukhija, B. S., Reddy, D. V., Nagabhushanam, P. Isotopic fingerprints of paleoclimates during the last 30,000 years in } \\
\text { deep confined groundwaters of Southern India. Quaternary Research, 50, 252-260 (1998). }\end{array}$ \\
\hline 142 & $\begin{array}{l}\text { Sukhija, B. S., Reddy, D. V., Nagabhushanam, P., Bhattacharya, S. K., Jani, R. A., Kumar, D. Characterisation of } \\
\text { recharge processes and groundwater flow mechanisms in weathered-fractured granites of Hyderabad (India) using } \\
\text { isotopes. Hydrogeology Journal, 14, 663-674 (2006). }\end{array}$ \\
\hline 143 & $\begin{array}{l}\text { Sultan, M., Sturchio, N. C., Gheith, H., Abdel Hady, Y., El Anbeawy, M. Chemical and isotopic constraints on the } \\
\text { origin of Wadi El-Tarfa ground water, eastern desert, Egypt. Ground water, 38, 743-751 (2000). }\end{array}$ \\
\hline 144 & $\begin{array}{l}\text { Sultan, M., Yan, E., Sturchio, N. C., Wagdy, A., Abdel Gelil, K., Becker, R., Manocha, N., Mileski, A. Natural } \\
\text { discharge: A key to sustainable utilization of fossil groundwater. Journal of Hydrology, 335, 25-36 (2007). }\end{array}$ \\
\hline 145 & $\begin{array}{l}\text { Sultan, M., Yan, E., Sturchio, N., Wagdy, A., Abdel Gelil, K., Becker, R., N. Manocha, N. Milewski, A., Natural } \\
\text { discharge: A key to sustainable utilization of fossil groundwater. Journal of Hydrology, 335, 25-35 (2007). }\end{array}$ \\
\hline 146 & $\begin{array}{l}\text { Takahashi, H. A., Nakamura, T., Tsukamoto, H., Kazahaya, K., Handa, H., Hirota, A. Radiocarbon dating of } \\
\text { groundwater in granite fractures in Abukuma Province, northeast Japan. Radiocarbon, 55, 894-904 (2013). }\end{array}$ \\
\hline 147 & $\begin{array}{l}\text { Talma, A. S., Weaver, J. M. C., Plummer, L. N., Busenburg, E. CFC tracing of groundwater in fractured rock aided } \\
\text { with 14C and }{ }^{3} \mathrm{H} \text { to identify water mixing. (eds. Sililo et al.) Groundwater: Past achievements and future challenges, } \\
\text { Balkema, Rotterdam (2000). }\end{array}$ \\
\hline 148 & $\begin{array}{l}\text { Taupin, J-D., Toro, L. E., Vargas, M. C. Etude géochimique et isotopique des aquifères dans la zone de Maicao } \\
\text { (Colombie). Hydrological Sciences-Journal-des Sciences Hydrologiques, 54, 538-555 (2009). }\end{array}$ \\
\hline 149 & $\begin{array}{l}\text { Taylor, C. B. Hydrology of the Poverty Bay flats aquifers, New Zealand: recharge mechanisms, evolution of the } \\
\text { isotopic composition of dissolved inorganic carbon, and ground-water ages. Journal of Hydrology, 158, 151-185 } \\
\text { (1994). }\end{array}$ \\
\hline 150 & $\begin{array}{l}\text { Taylor, C. B., Trompetter, V. J., Brown, L. J., Bekesi, G. The Manawatu aquifers, North Island, New Zealand: } \\
\text { Clarification of hydrogeology using a multidisciplinary environmental tracer approach. Hydrological Processes, 15, } \\
\text { 3269-3286 (2001). }\end{array}$ \\
\hline 151 & $\begin{array}{l}\text { Thiros, S. Quality and sources of shallow ground water in areas of recent residential development in Salt Lake Valley, } \\
\text { Salt Lake County, Utah. US Geological Survey Water-Resources Investigations Report 03-4028, 277, 214-226 (2010). }\end{array}$ \\
\hline
\end{tabular}




\begin{tabular}{|c|c|}
\hline \# & eference \\
\hline 152 & $\begin{array}{l}\text { Toro, L. E., Calderon, J., Taupin, J. D., Vargas Quintero, M. C. Exploración de aguas subterráneas en Maicao } \\
\text { (Colombia) mediante técnicas hidroquímicas e isotópicas. in: Estudios de Hidrología Isotópica en América Latina, } \\
\text { IAEA-TECDOC-1611, 67-82 (2008). }\end{array}$ \\
\hline 153 & $\begin{array}{l}\text { Trabelsi, R., Abid, K., Zouari, K. Geochemistry processes of the Djeffara palaeogroundwater (Southeastern Tunisia). } \\
\text { Quaternary International, 257, 43-55 (2012). }\end{array}$ \\
\hline 154 & $\begin{array}{l}\text { Trettin, R., Freyer, K., Strauch, G., Treutler, H. C., Dehnert, J., Nestler, W., Grischek, T. Isotope studies to reveal } \\
\text { groundwater origin and flow processes within the drinking water supply area Elbe/Torgau. in: Isotopes in water } \\
\text { resources management, Vol. 1., International Atomic Energy Agency, Vienna, Austria, 457-459 (1995). }\end{array}$ \\
\hline 155 & $\begin{array}{l}\text { Trettin, R., Knoller, K., Loosli, H. H., Kowski, P. Evaluation of the sulfate dynamics in groundwater by means of } \\
\text { environmental isotopes. Isotopes in Environmental and Health Studies, 38, 103-119 (2002). }\end{array}$ \\
\hline 156 & $\begin{array}{l}\text { Visser, A., Broers, H. P., Purtschert, R., Sültenfuß, J., de Jonge, M. Groundwater age distributions at a public drinking } \\
\text { water supply well field derived from multiple age tracers ( } 85 \mathrm{Kr}, 3 \mathrm{H} / 3 \mathrm{He} \text {, and 39Ar). Water Resources Research, 49, } \\
7778-7796 \text { (2013). }\end{array}$ \\
\hline 157 & $\begin{array}{l}\text { Vogel, J. C., Lerman, J. C., Mook, W. G., \& Roberts, F. B. Natural isotopes in the groundwater of the Tulum Valley, } \\
\text { San Juan, Argentina. Hydrological Sciences Journal, 17, 85-96 (1975). }\end{array}$ \\
\hline 158 & $\begin{array}{l}\text { Wang, L., Hu, F., Yin, L., Wan, L., Yu, Q. Hydrochemical and isotopic study of groundwater in the Yinchuan plain, } \\
\text { China. Environmental and Earth Sciences, 69, 2037-2057 (2013). }\end{array}$ \\
\hline 159 & $\begin{array}{l}\text { Wang, S. Hydrochemical and isotopic characteristics of groundwater in the Yanqi Basin of Xinjiang province, } \\
\text { northwest China. Environmental and Earth Sciences, } 71,427-440(2014) \text {. }\end{array}$ \\
\hline 160 & $\begin{array}{l}\text { Wang, Y., Jiao, J. J. Origin of groundwater salinity and hydrogeochemical processes in the confined Quaternary aquifer } \\
\text { of the Pearl River Delta, China. Journal of Hydrology, } 438-439,112-124(2012) \text {. }\end{array}$ \\
\hline 161 & $\begin{array}{l}\text { Weyhenmeyer, C. E., Burns, S. J., Waber, H. N., Aescl } \\
\text { glacial temperatures and changes in moisture source re }\end{array}$ \\
\hline 162 & $\begin{array}{l}\text { Yangui, H., Abidi, I., Zouari, K., Rozanski, K. Deciphering groundwater flow between the Complex Terminal and Plio- } \\
\text { Quaternary aquifers in Chott Gharsa plain (southwestern Tunisia) using isotopic and chemical tools. Hydrological } \\
\text { Sciences Journal, 57, 967-984 (2012). }\end{array}$ \\
\hline 163 & $\begin{array}{l}\text { Yangui, H., Zouari, K., Rozanski, K. Hydrochemical and isotopic study of groundwater in Wadi El Hechim-Garaa } \\
\text { Hamra basin, Central Tunisia. Environmental Earth Science, 66, 1359-1370 (2012). }\end{array}$ \\
\hline 164 & $\begin{array}{l}\text { Yangui, H., Zouari, K., Trabelsi, R., Rozanski, K. Recharge mode and mineralization of groundwater in a semi-arid } \\
\text { region: Sidi Bouzid plain (central Tunisia). Environmental Earth Sciences, 63, 969-979 (2011). }\end{array}$ \\
\hline 165 & $\begin{array}{l}\text { Yechieli, Y., Kafri, U., Sivan, O. The inter-relationship between coastal sub-aquifers and the Mediterranean Sea, } \\
\text { deduced from radioactive isotopes analysis. Hydrogeology Journal, 17, 264-274 (2008). }\end{array}$ \\
\hline 166 & $\begin{array}{l}\left.\text { Yin, L., Hou, G., Su, Xs., Wang, D., Dong, J., Hao, Y., Wang, X. Isotopes ( } \delta \mathrm{D} \text { and } \delta^{18} \mathrm{O}\right) \text { in precipitation, groundwater } \\
\text { and surface water in the Ordos Plateau, China: implications with respect to groundwater recharge and circulation. } \\
\text { Hydrogeology Journal, 19, 429-443 (2010). }\end{array}$ \\
\hline 167 & $\begin{array}{l}\text { Yitbarek, A., Razack, M., Ayenew, T., Zemedagegnehu, E., Azagegn, T. Hydrogeological and hydrochemical } \\
\text { framework of Upper Awash River basin, Ethiopia: With special emphasis on inter-basins groundwater transfer between } \\
\text { Blue Nile and Awash Rivers. Journal of African Earth Sciences, 65, 46-60 (2012). }\end{array}$ \\
\hline 168 & $\begin{array}{l}\text { Zhang, B., Song, X., Zhang, Y., Han, D., Tang, C., Yang, L., Wang, Z., Liu, T. A study of the interrelation between } \\
\text { surface water and groundwater using isotopes and chlorofluorocarbons in Sanjiang plain, Northeast China. } \\
\text { Environmental Earth Sciences, 72, 3901-3913 (2014). }\end{array}$ \\
\hline 169 & ional groundwater data for the USA was obtained from Water Quality Portal web page: www.waterqualitydata.us \\
\hline
\end{tabular}


Table S3. Industrial, agricultural, domestic or municipal well construction purposes

\begin{tabular}{|c|c|}
\hline State & Well construction purposes included in our analysis shown in Figure 3 \\
\hline California & $\begin{array}{l}\text { [Other Recreation] [Water Supply Irrigation - Landscape ] [Water Supply Domestic ] [Water Supply } \\
\text { Public ] [Other Fire or Frost Protection] [Other Bottled Water] [Other Power Generation] [Water Supply } \\
\text { Industrial] [Other Dairy Supply] [Other iriigation] [Other Irrigation] [Water Supply Irrigation - } \\
\text { Agriculture] [Water Supply Stock or Animal Watering] [Water Supply Irrigation Agricultural] }\end{array}$ \\
\hline Colorado & 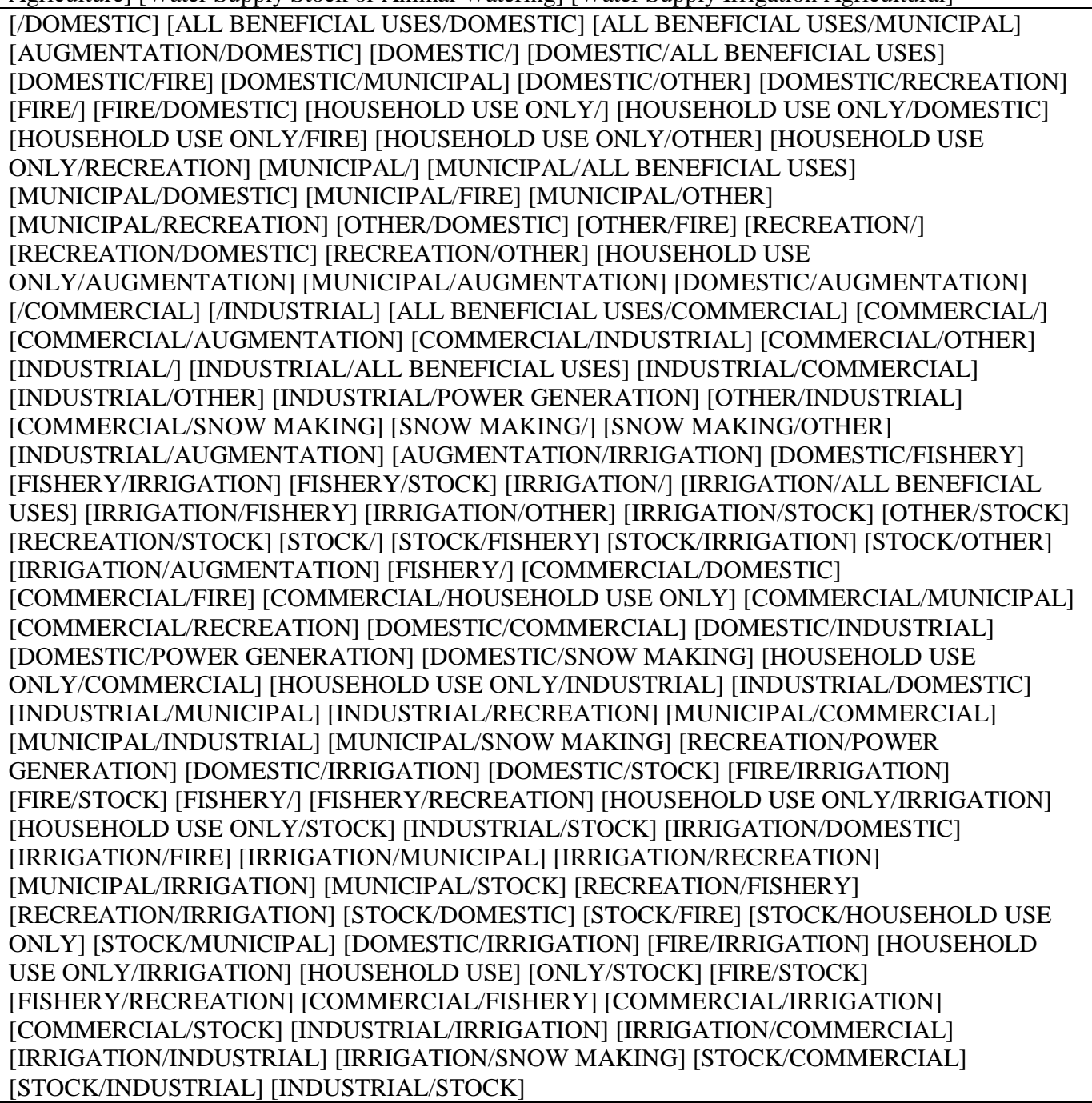 \\
\hline Kansas & $\begin{array}{l}\text { [Pond/Swimming Pool/Recreation] [Public Water Supply] [Lawn and Garden - domestic only] [Domestic] } \\
\text { [Domestic, changed from Irrigation] [Domestic, changed from Oil Field Water Supply] [Industrial] [Oil } \\
\text { Field Water Supply] [Irrigation] [Feedlot] [Feedlot/Livestock/Windmill] [Domestic, Livestock] }\end{array}$ \\
\hline Nebraska & [Domestic] [Commercial/Industrial] [Aquaculture] [Pit - Irrigation] [Irrigation] [Livestock] \\
\hline Wyoming & $\begin{array}{l}\text { [DOM_GW] [MUN_GW] [DOM_GW; MIS] [DOM_GW;MIS] [DOM_GW; TST] [MIS; MUN_GW] } \\
\text { [DOM_GW; MUN_GW] [MIS; DOM_GW] [DOM_GW; MON] [IND_GW] [IND_GW; MIS] [IND_GW; } \\
\text { MON] [IND_GW;MIS] [IND_GW; MIS; MON] [IND_GW; MIS; MIS] [MIS; IND_GW] [STK] [MIS; } \\
\text { STK] [IRR_GW] [IRR_GW; STK] [STK; IRR_GW] [MON; STK] [IRR_GW; MIS] [MIS; MON; STK] } \\
\text { [IRR_GW;MIS] [IRR_GW; MIS; STK] [MIS; IRR_GW] [STK; MIS] [IRR_GW;STK] [STK; MIS; MIS] } \\
\text { [MIS; STK; IRR_GW] [MIS;STK] [STK; IRR_GW; MIS] [IRR_GW; MIS; MIS; MIS; MIS] } \\
\text { [IRR_GW;MIS;STK] [DOM_GW; IND_GW] [DOM_GW; IND_GW; MIS] [DOM_GW; IND_GW; } \\
\text { MUN_GW] [IND_GW; MUN_GW] [IND_GW; MIS;MUN_GW] }\end{array}$ \\
\hline
\end{tabular}




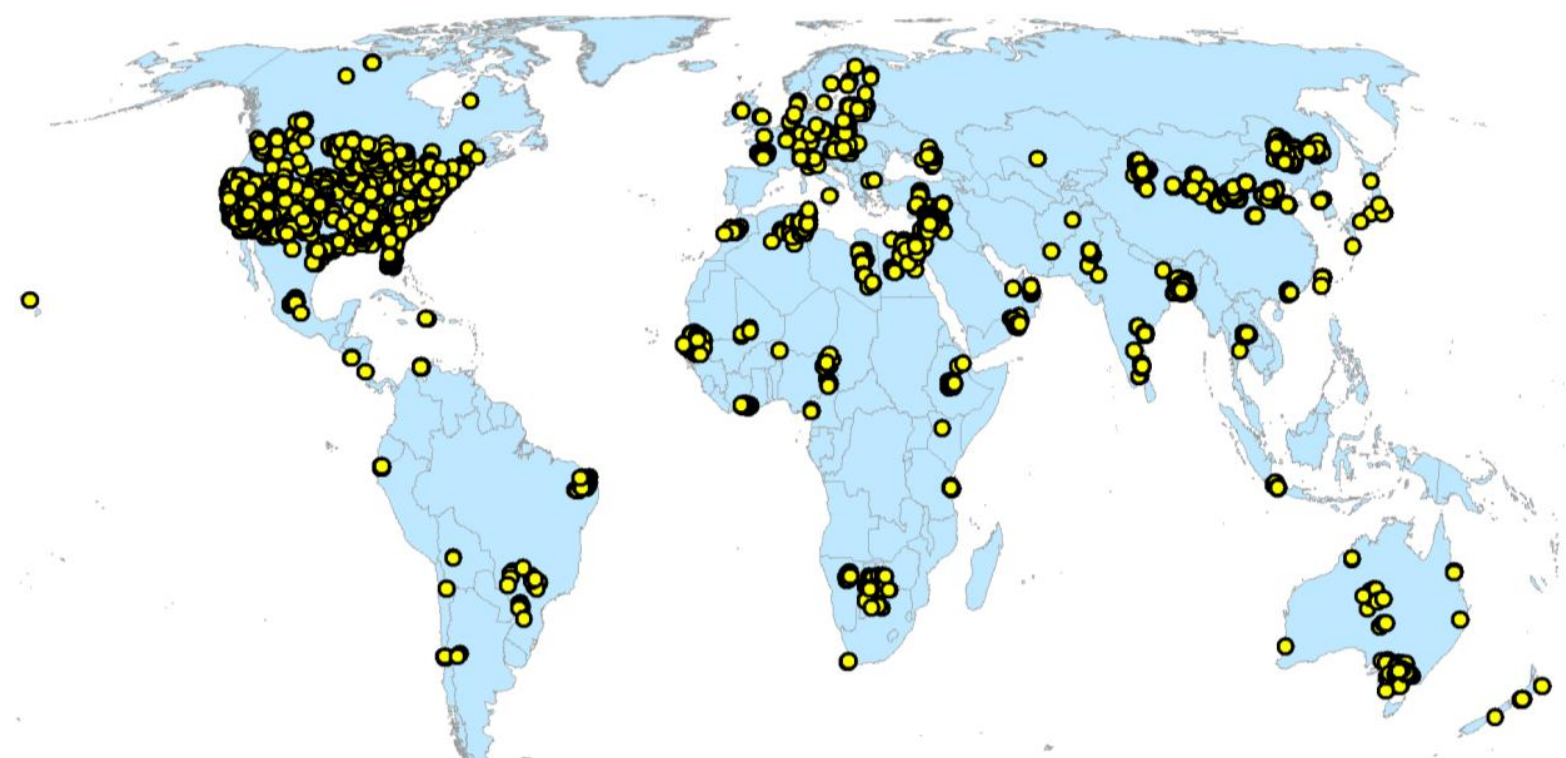

Figure S1. Locations of the analysed well waters with tritium and/or radiocarbon data (yellow circles). Outlines of countries are marked by grey lines. 


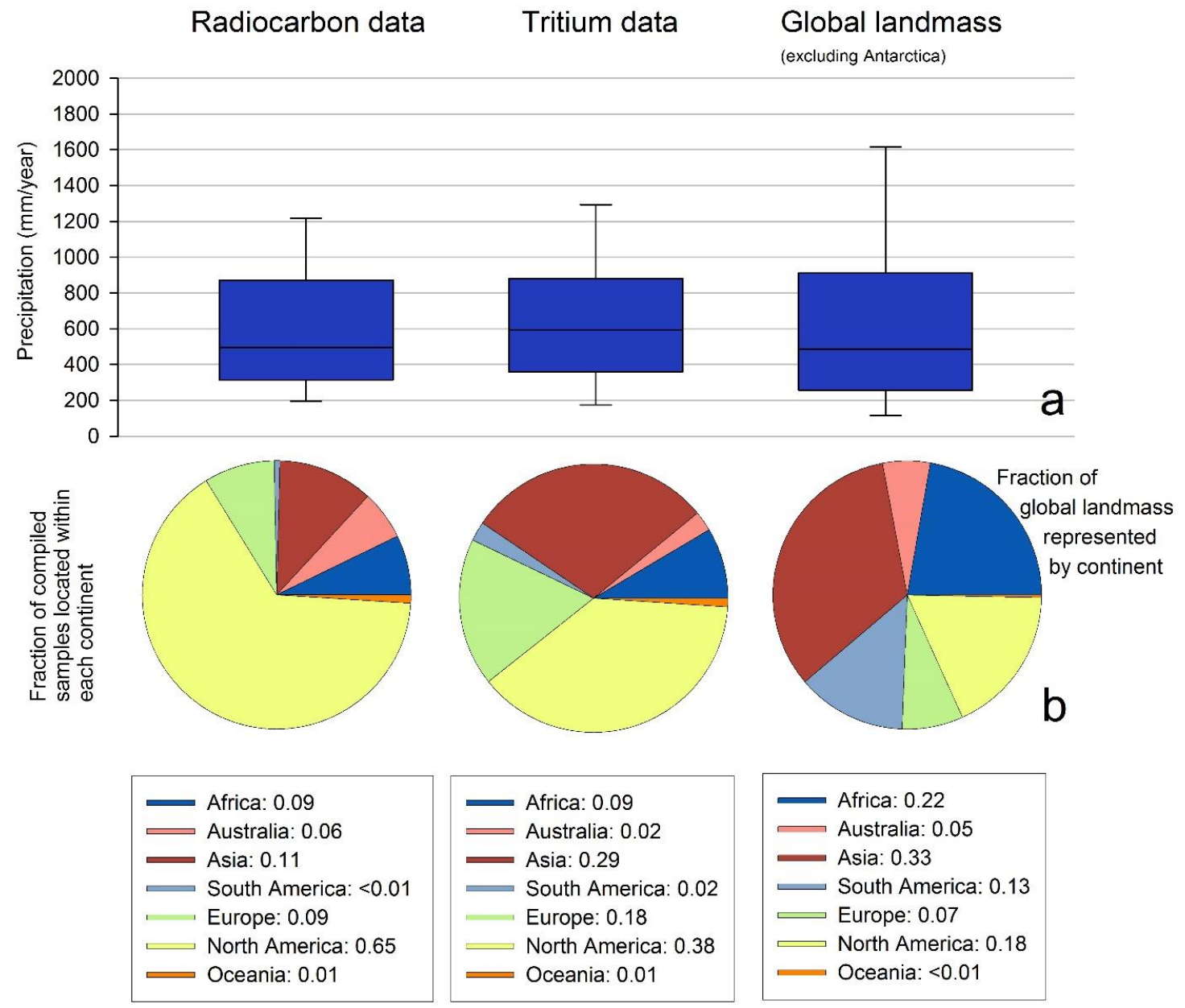

Figure S2. Sampling biases of radiocarbon (left column) and tritium (middle column), relative to the distribution of the global landmass by continent (right column). Panel (a) shows a boxwhisker plot of annual precipitation ( $\mathrm{mm} / \mathrm{year}$ ) at the wells where we compiled radiocarbon data (leftmost plot), the wells where we compiled tritium data (middle plot), and the entire global landmass excluding Antarctica (rightmost plot; data from Ref. 45). Panel (b) shows the fraction of all radiocarbon (leftmost pie chart) and tritium (middle pie chart) measurements that we compiled, juxtaposed against the distribution of landmass categorized by continental area (rightmost plot). Our dataset disproportionately represents some continents relative to their land areas (e.g., North America, yellow above). Our compiled data represent many climate zones and generally represents the global distribution of precipitation rates (panel a). However, we note that several continents are disproportionately represented in our compilation relative to the areas of the continents, specifically for North America, which accounts for $65 \%$ and $38 \%$ of compiled radiocarbon and tritium data, but only $18 \%$ of the global landmass. 


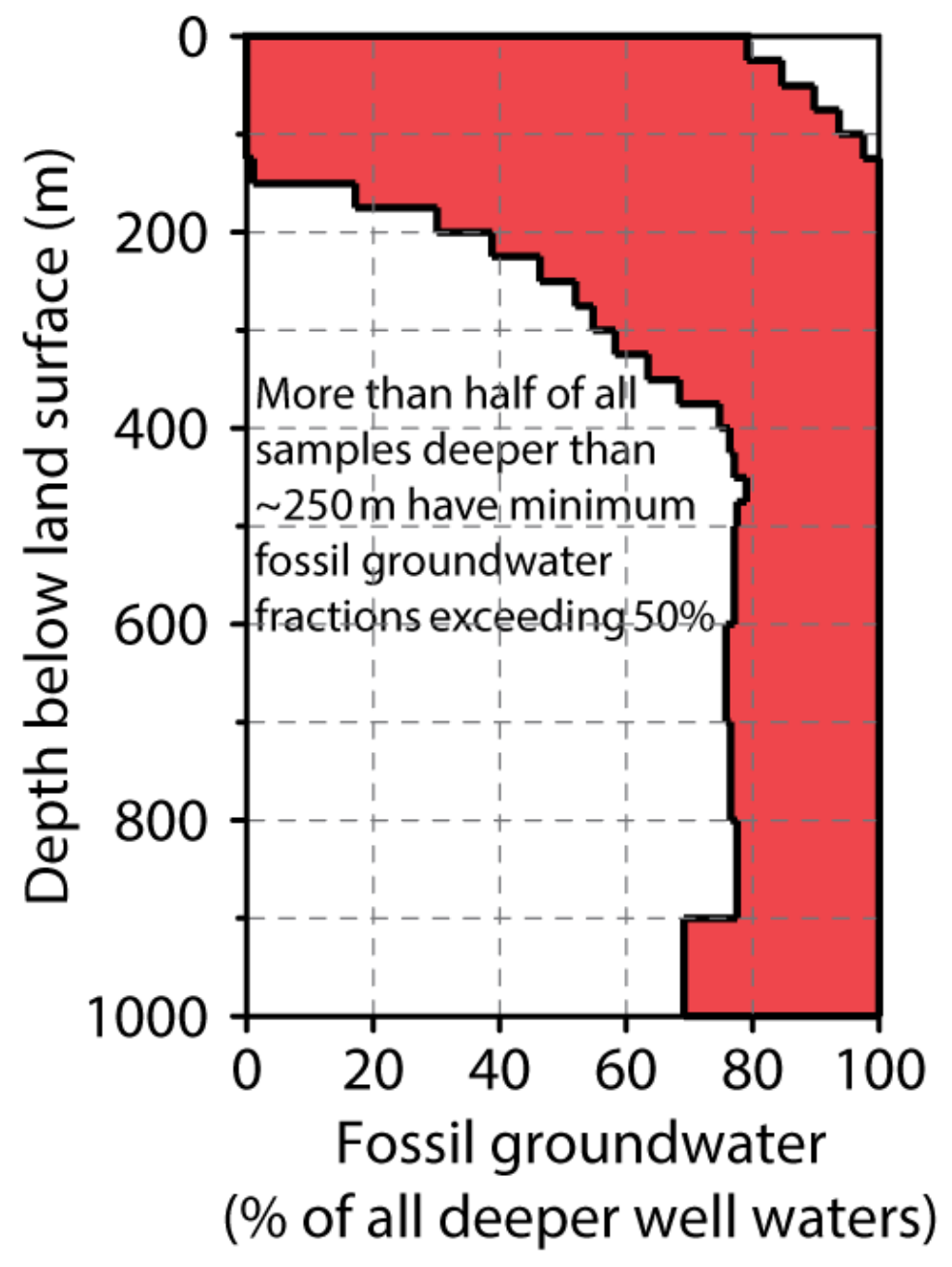

Figure S3. Depth variations of the fraction of fossil groundwater in all compiled underlying (i.e., deeper) wells. The figure presents the median value of the upper and lower (maximum and minimum) estimates of fossil and post-1953 groundwater as calculated using all underlying wells. More than half of all compiled well waters pumped from depths of deeper than $250 \mathrm{~m}$ below the land surface have a minimum fossil groundwater fraction of greater than $50 \%$. 


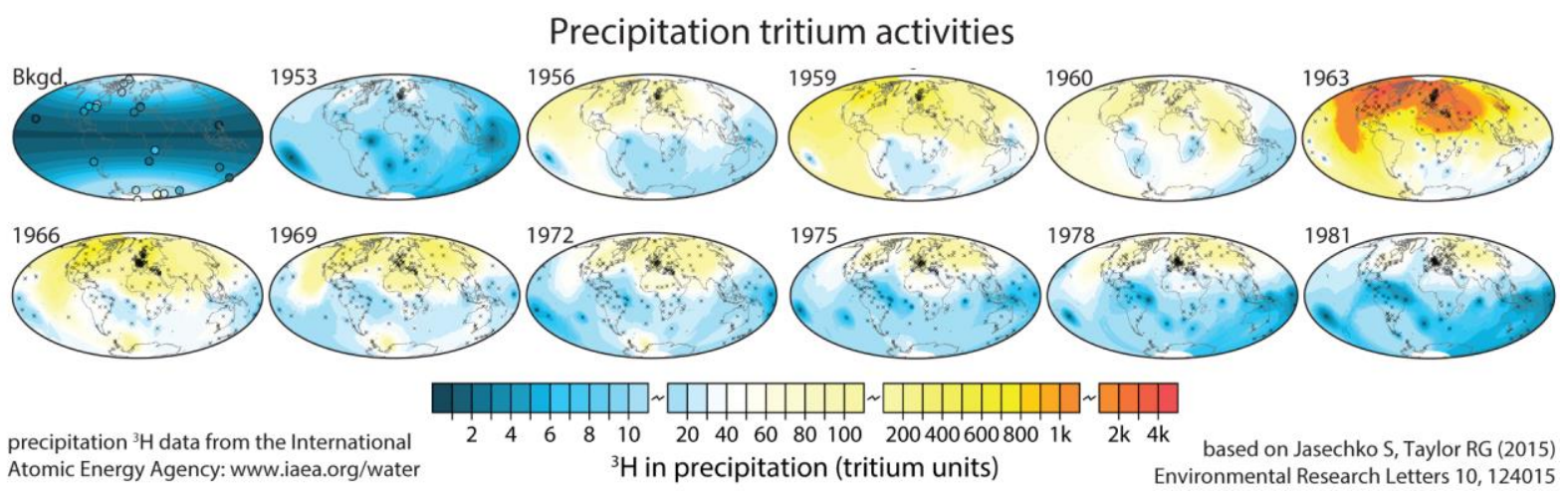

Figure S4. Time series of interpolated global precipitation tritium from pre-1953 (background: "Bkgd.") through the year 1981 (lower right plot). Precipitation tritium measurements are from the International Atomic Energy Agency's precipitation isotope database (www.iaea.org/water). The maps shown here are based on those from Ref. 24. 

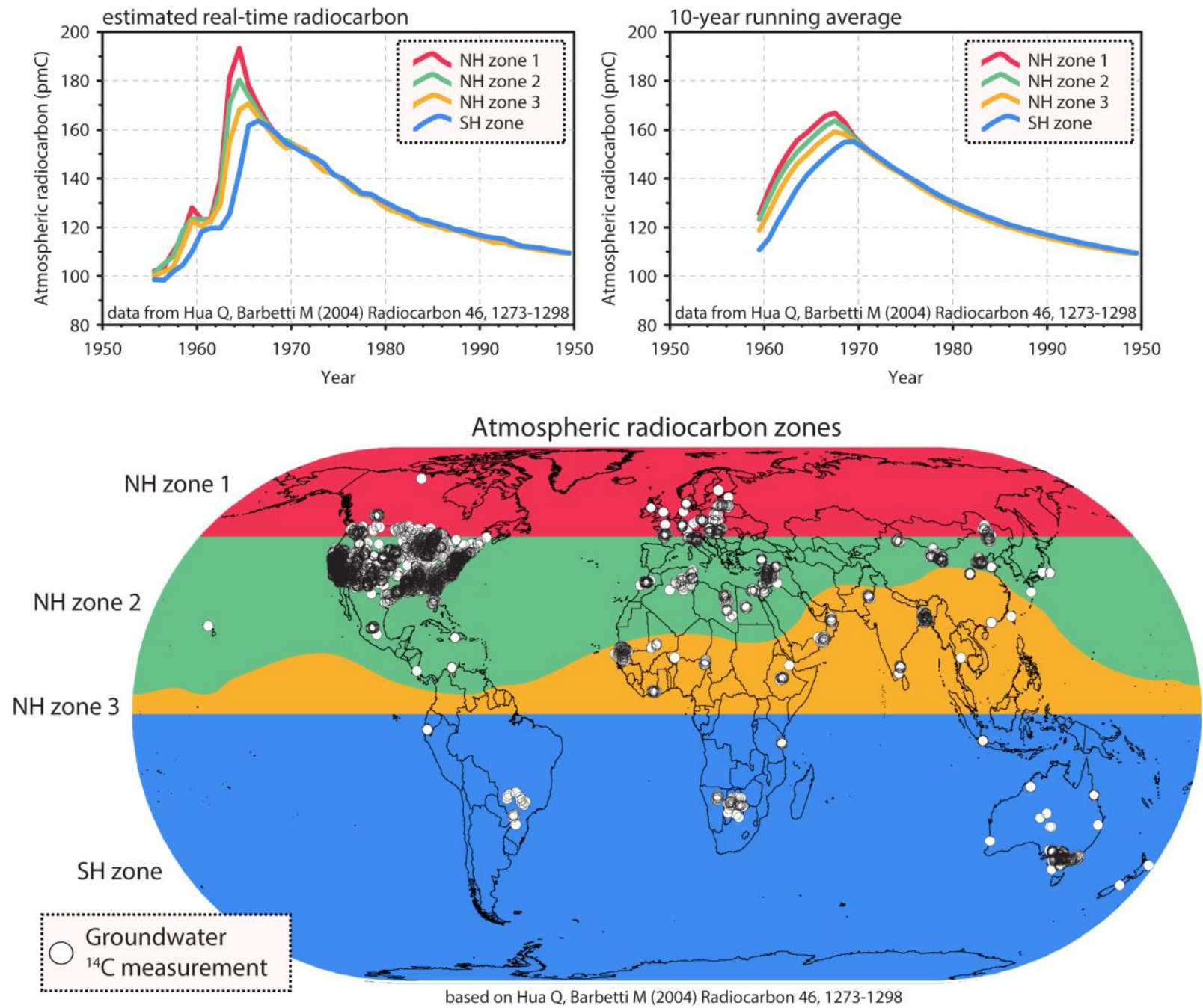

Figure S5. Atmospheric radiocarbon concentrations and groundwater 14C sampling locations. The upper two panels show estimated time series of atmospheric radiocarbon in three geographic zones (left panel), and a 10-year moving average of these atmospheric $14 \mathrm{C}$ data (right panel). That is, the data shown in the upper left panel are estimates of the real-time atmospheric radiocarbon content, and the line plots in the top right panel are 10-year moving averages of the data in the upper left panel. The lower map shows the locations of each of the radiocarbon zones, where the colours correspond to the colours of the lines in the upper two panels. The circles show groundwater well locations where fossil groundwater calculations were performed in our manuscript. The atmospheric radiocarbon time series and zones are based on Hua, Q. \& Barbetti, M. (2004). Radiocarbon 46, 1273-1298 (Ref. 43). 


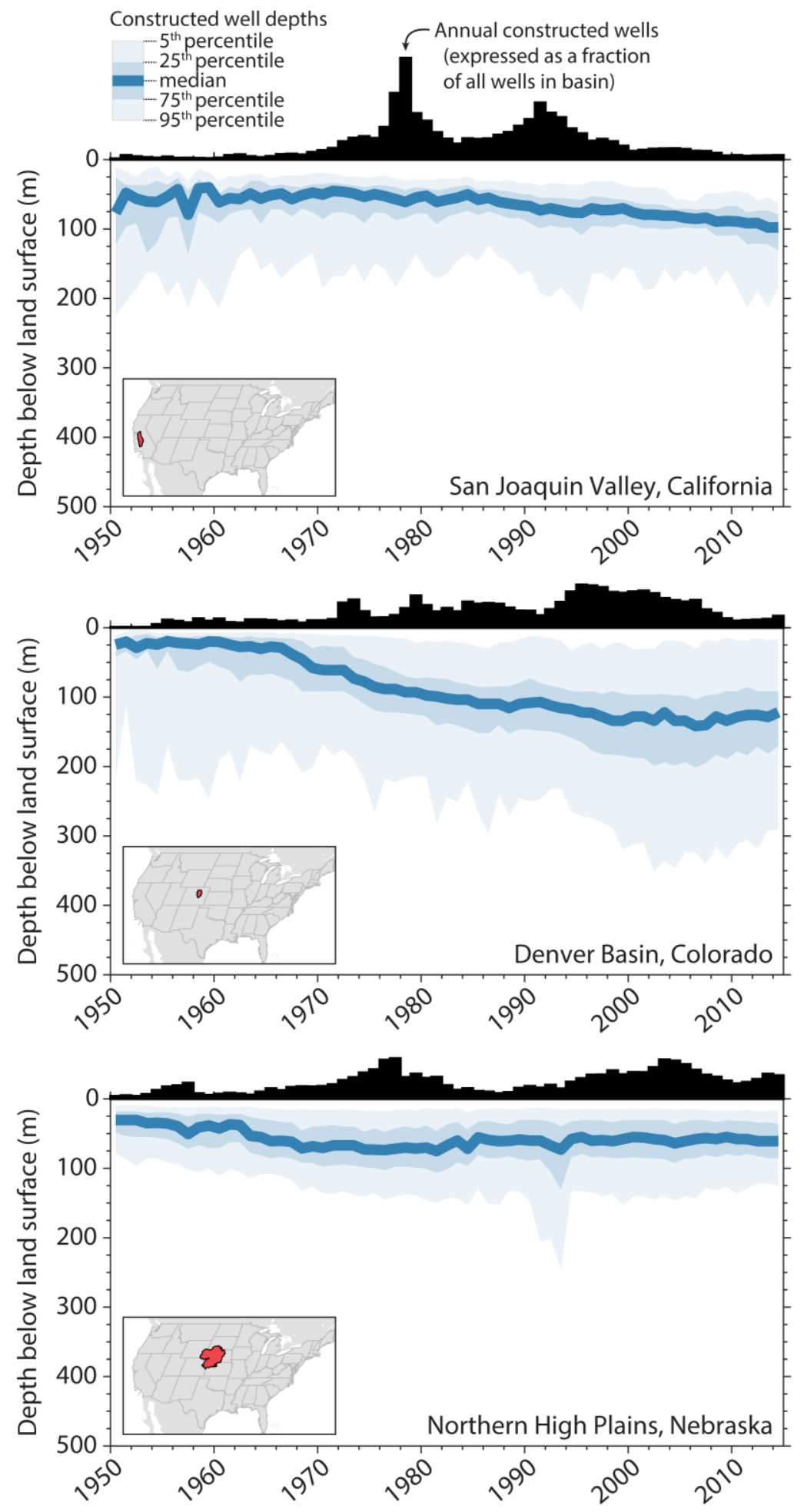

Figure S6. Groundwater well construction depth trends (19502015) in six western US aquifers. The blue line shows the annual median well construction depth, the medium blue shading marks the upper-lower quartile well depths, and the light blue shading marks the $5^{\text {th }}-95^{\text {th }}$ percentile range of wells. In the San Joaquin Valley and the Northern High Plains, constructed wells lie above the depth that these aquifer systems transition to mostlyfossil groundwater. In the Denver Basin, many wells are constructed at similar depths as fossil groundwaters. 
Figures S7 (following pages). For figure description see Supplementary Information section S2. 

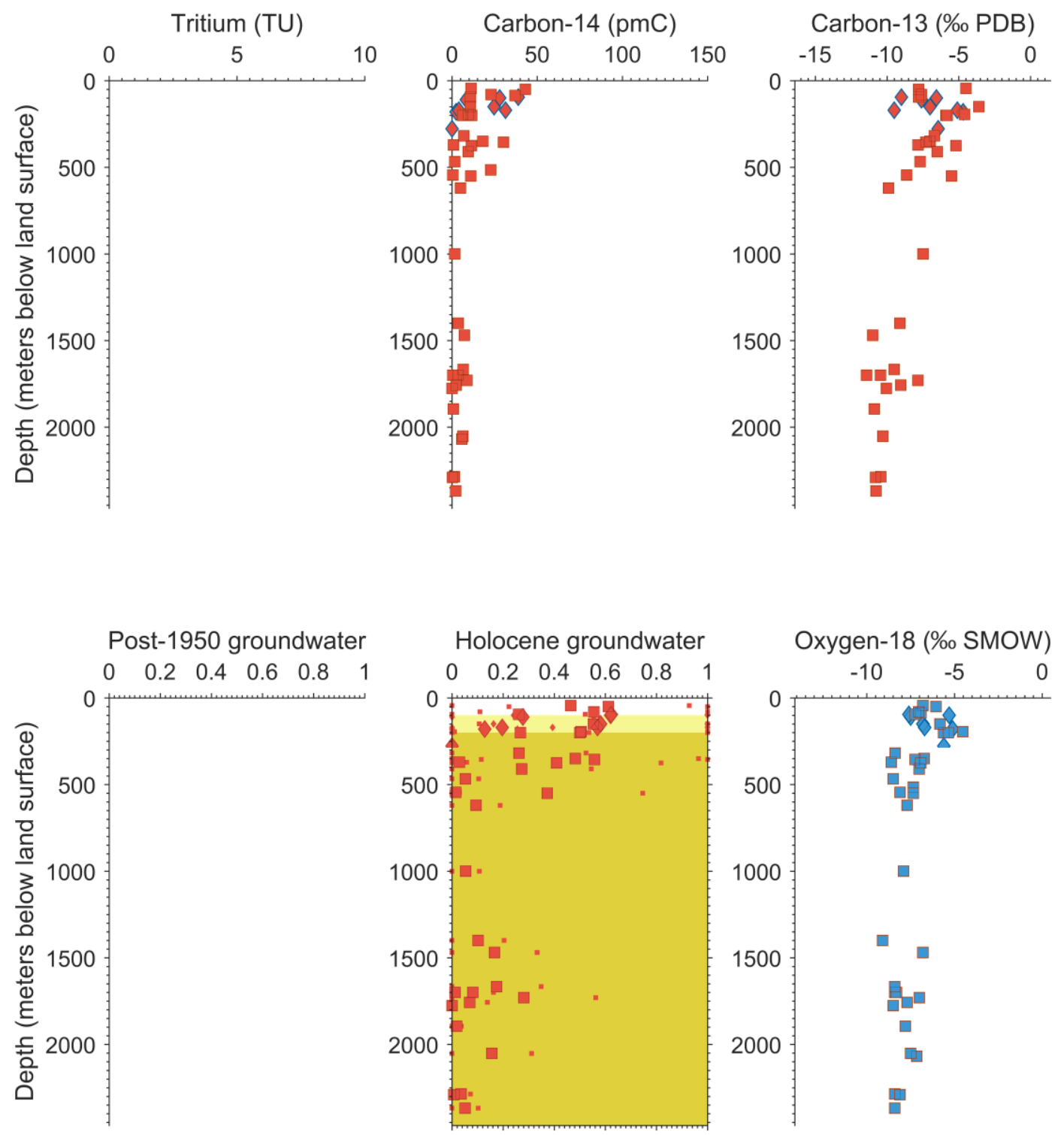

Great Oriental Erg: Continental Intercalaire, Algeria 

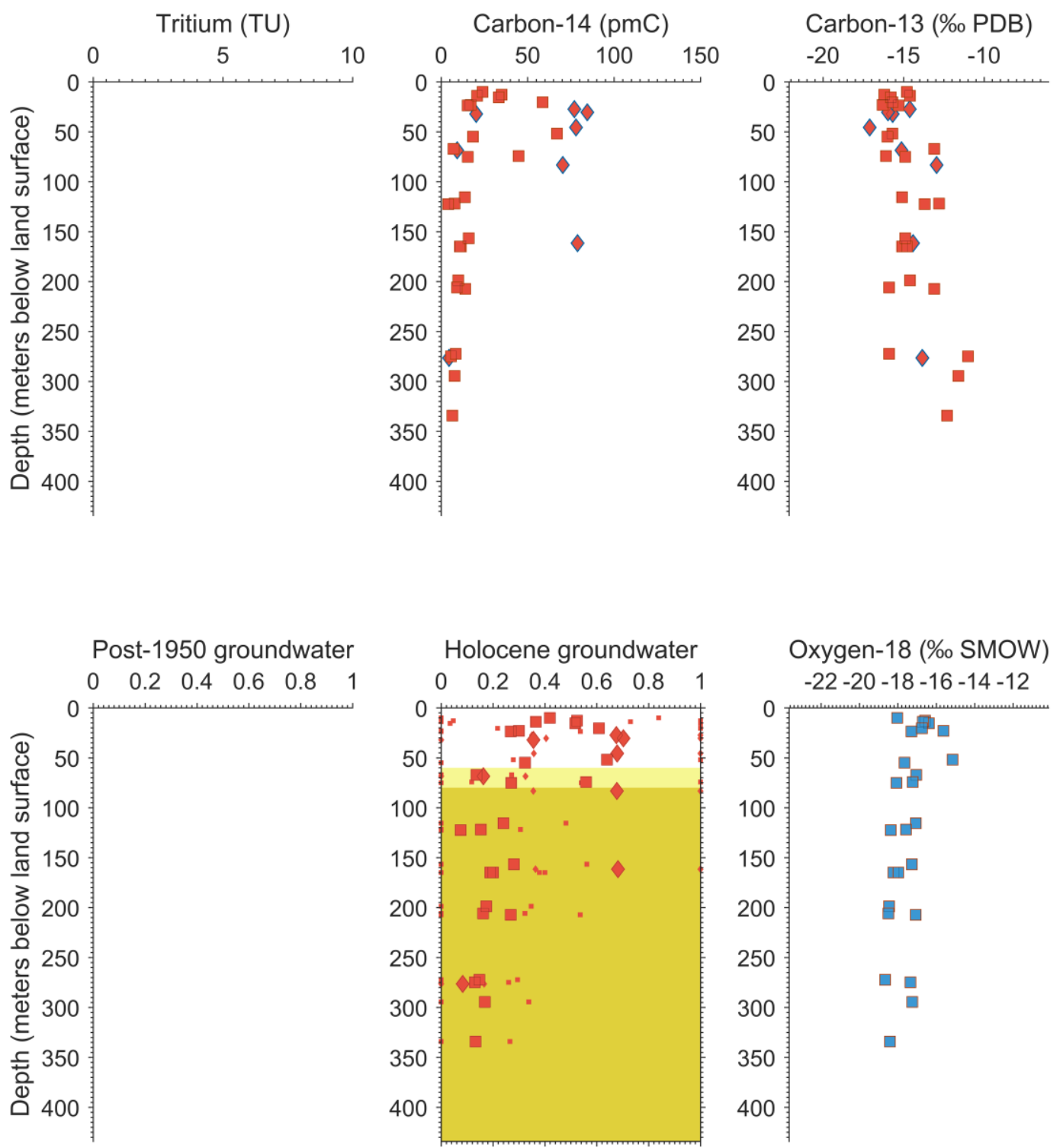

\section{Palouse Basin, USA}



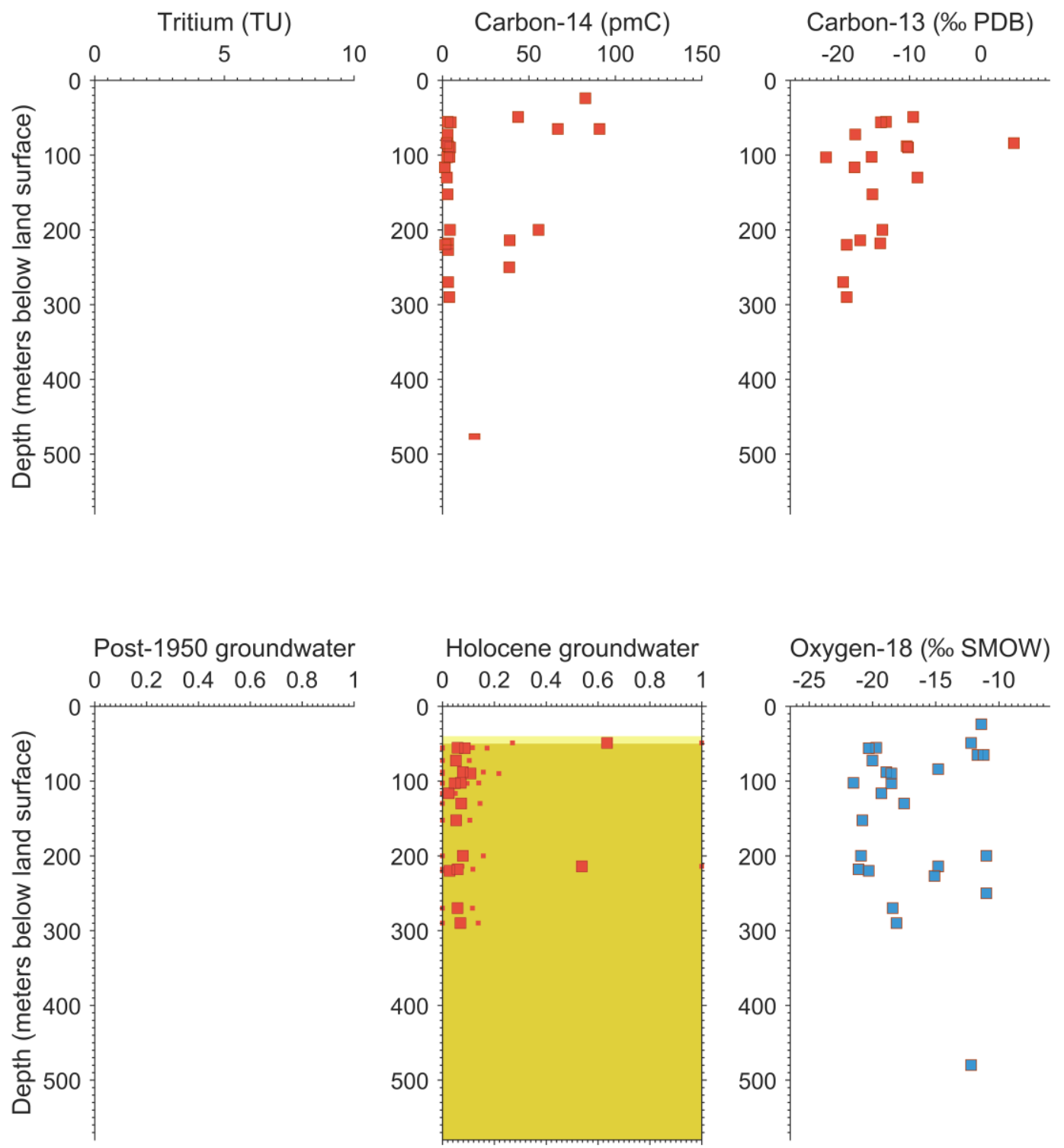

\section{Cambrian-Vendian Aquifer, Estonia}



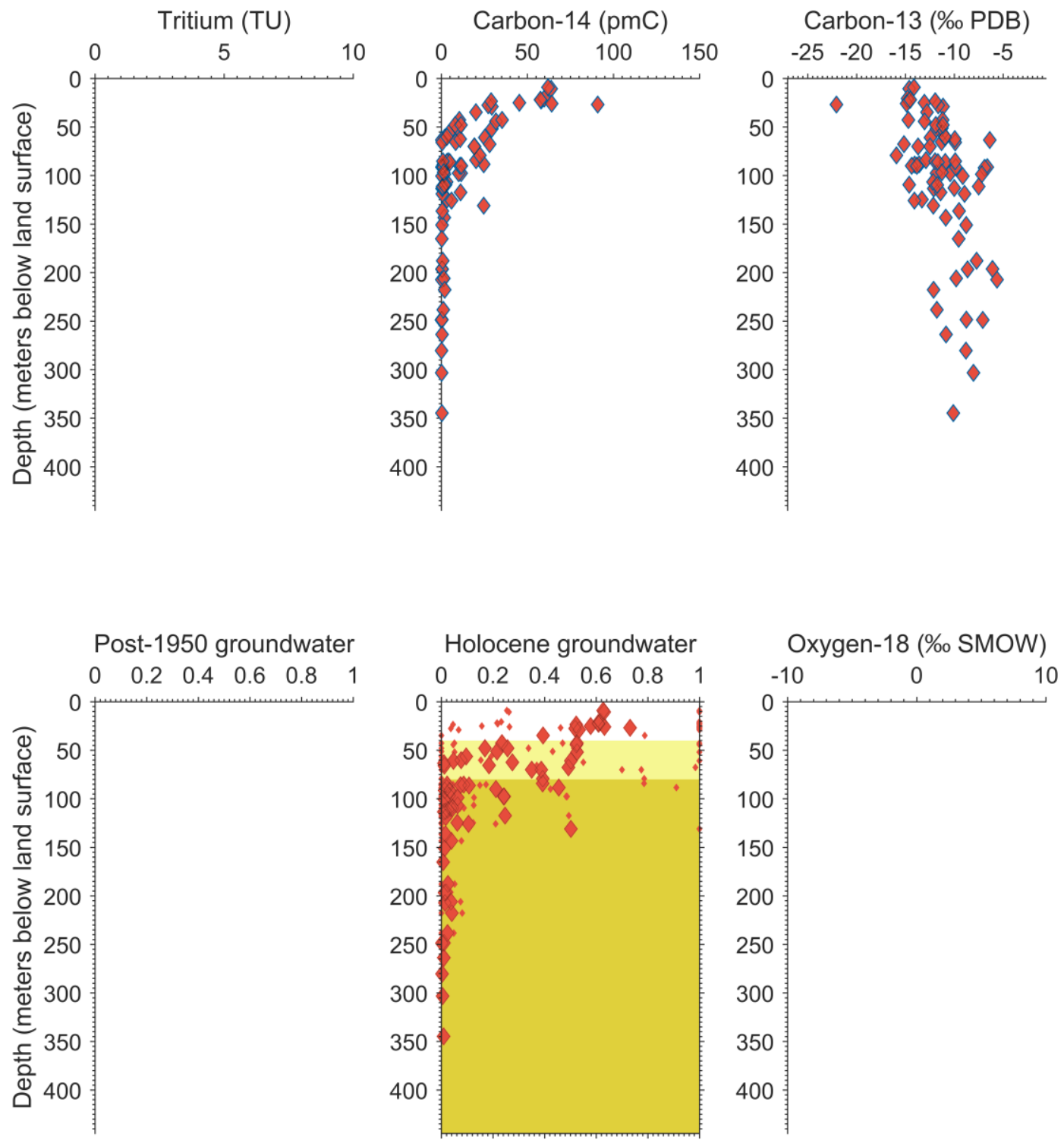

Northern Atlantic Coast, USA 

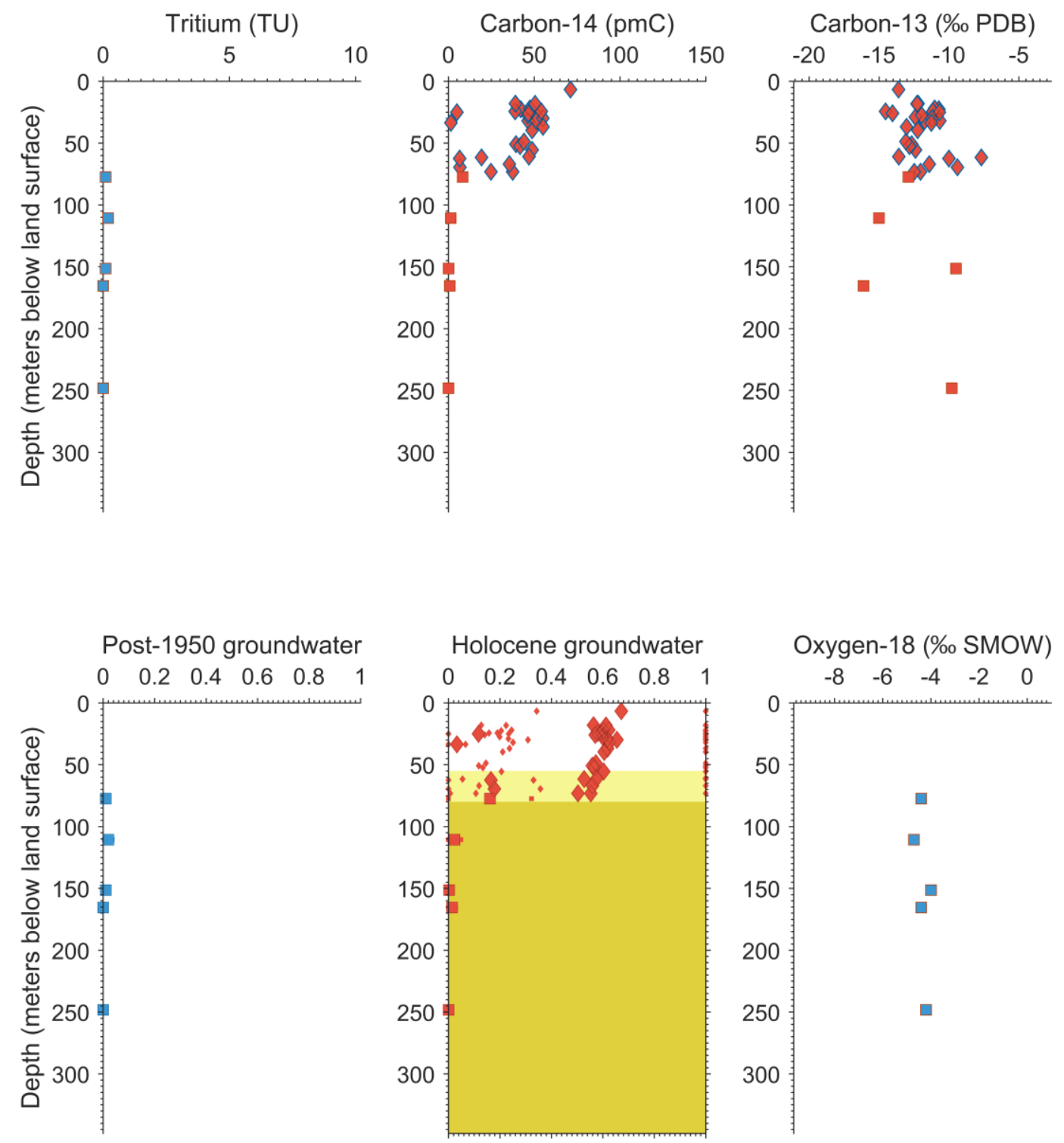

Castle Hayne Aquifer, USA 

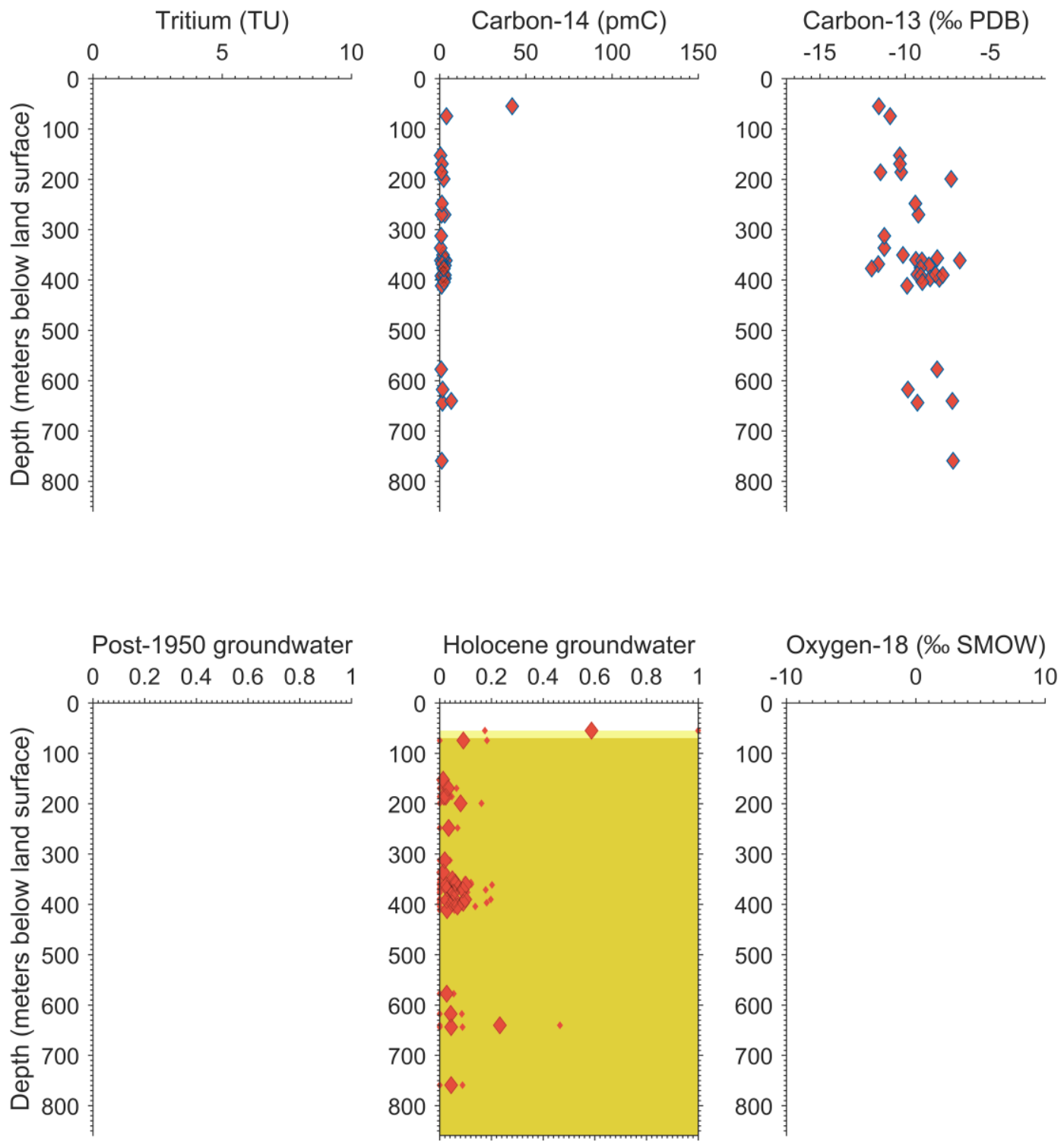

Jacksonville Aquifer, USA 

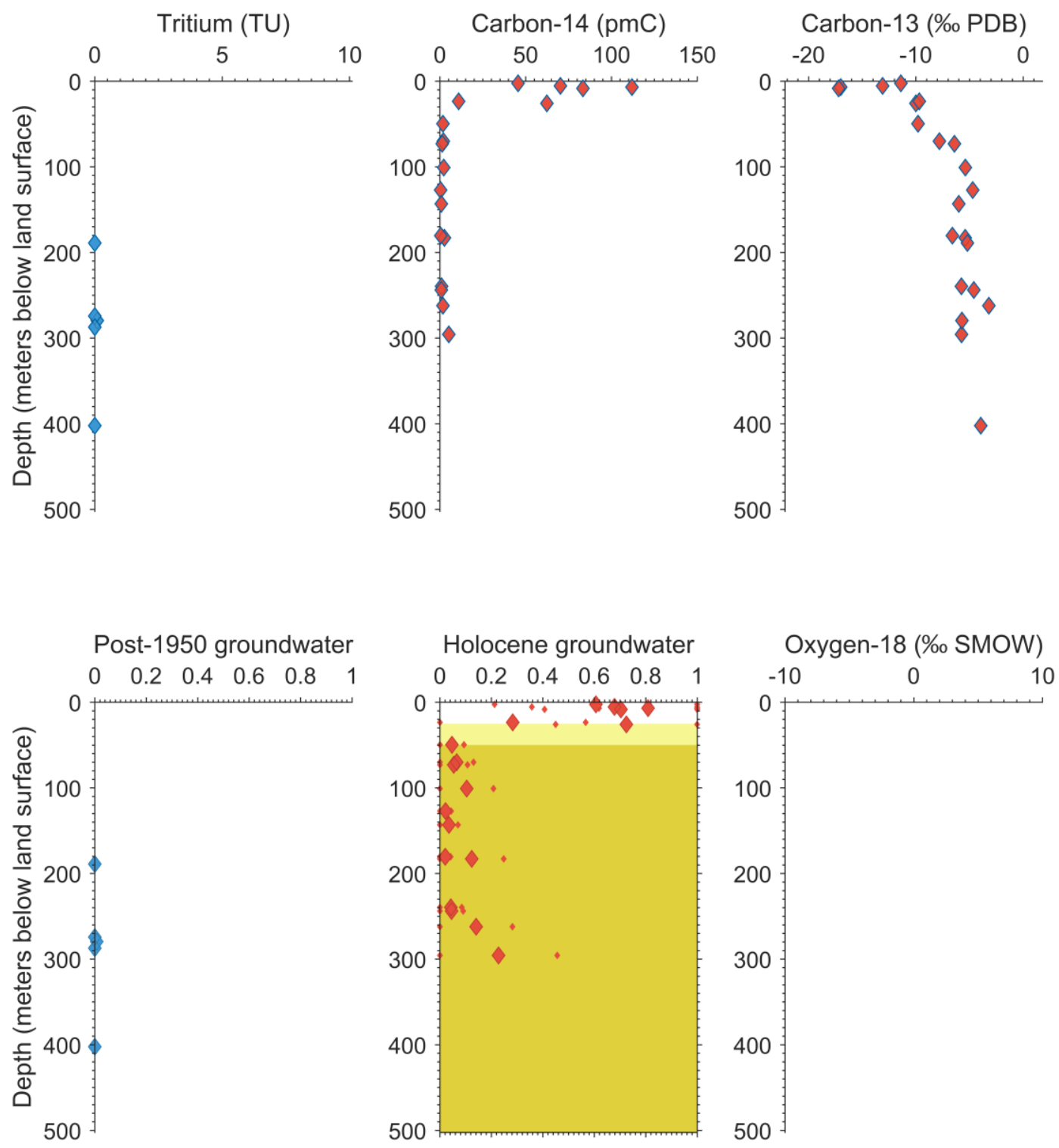

Tampa Bay Aquifer, USA 

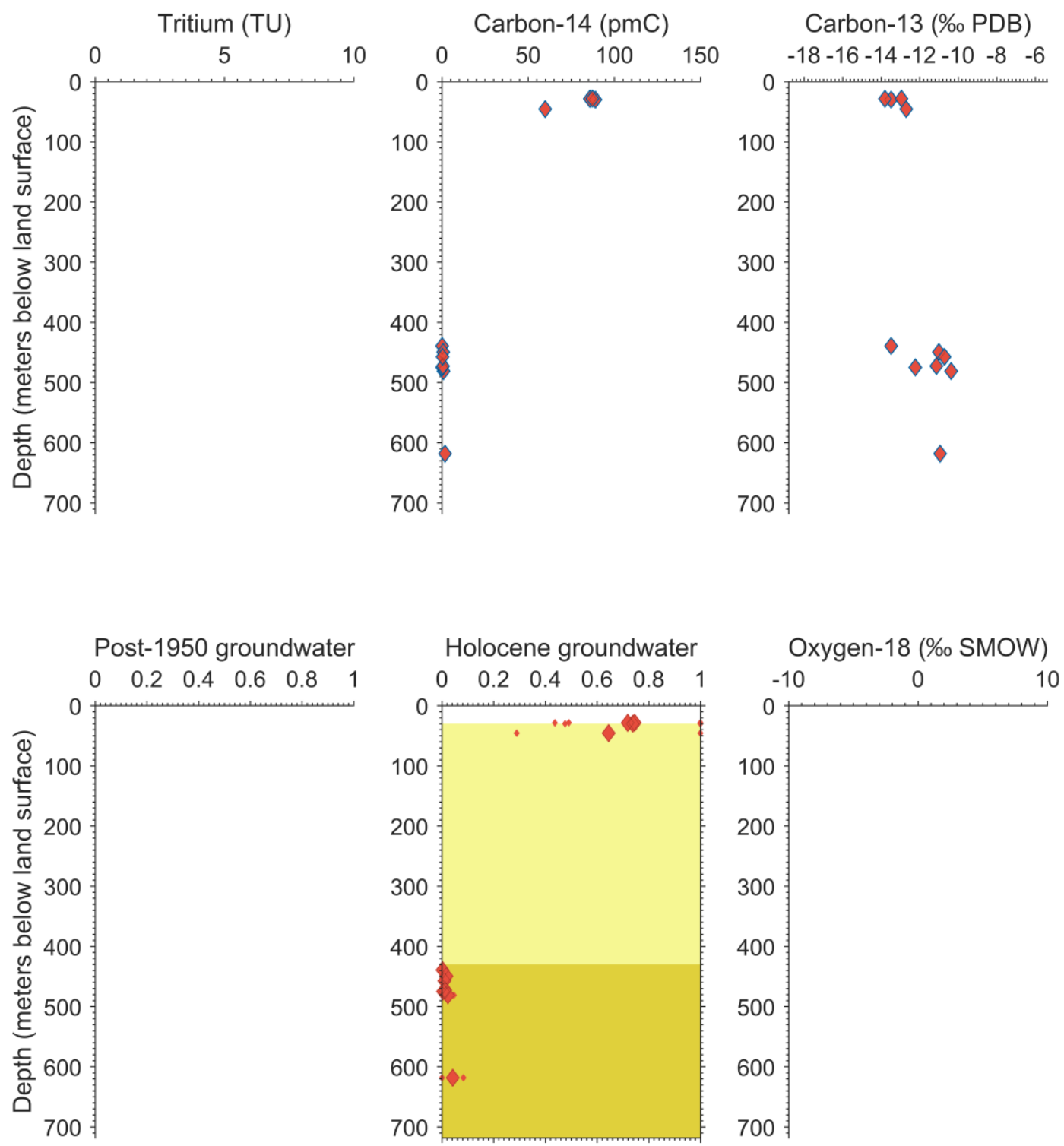

Silurian-Devonian Aquifer, USA 

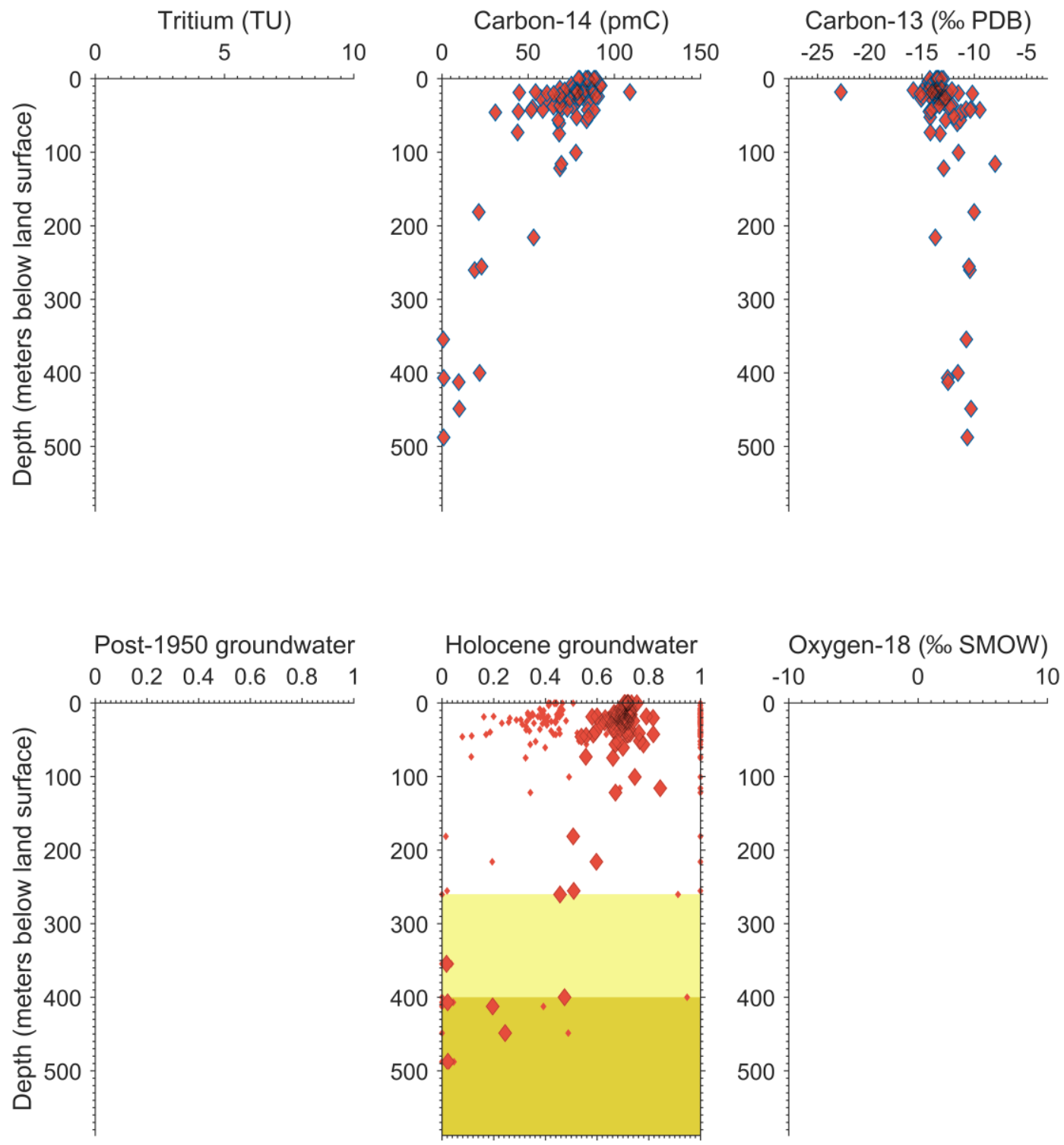

\section{Cambrian Ordovician Sandstone, USA}



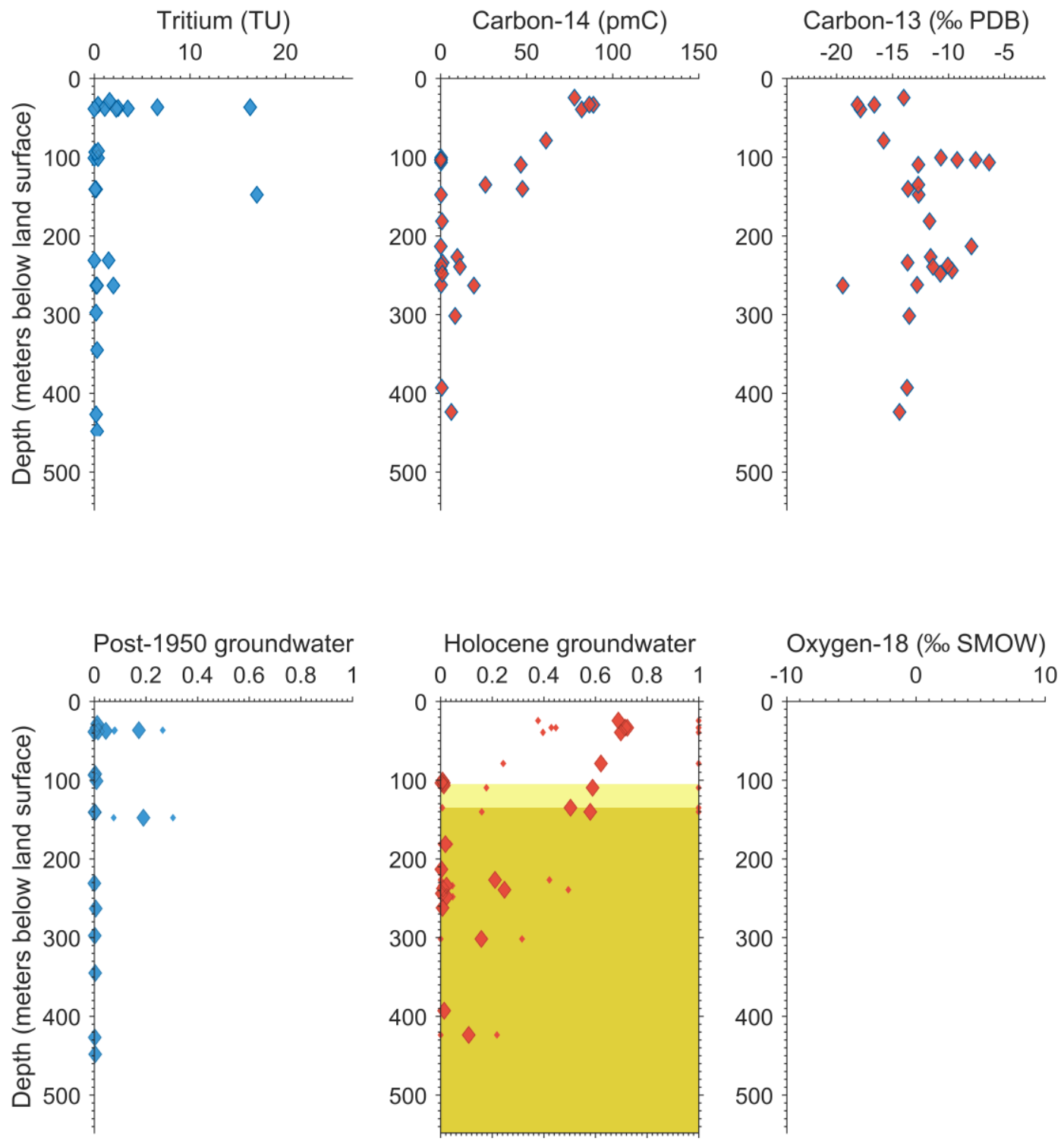

Mississippi Alluvial Aquifer, USA 

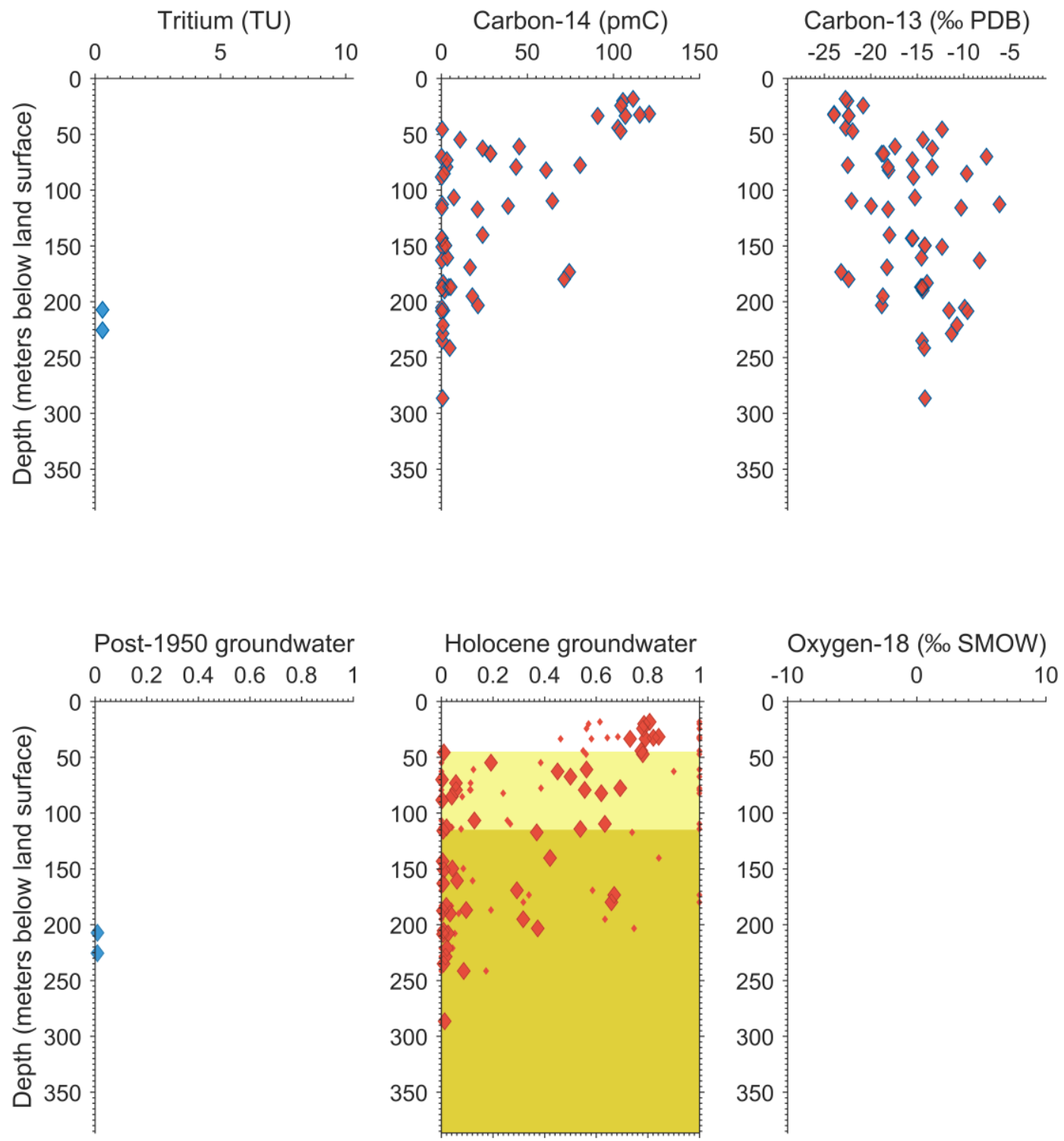

Mississippi Embayment, USA 

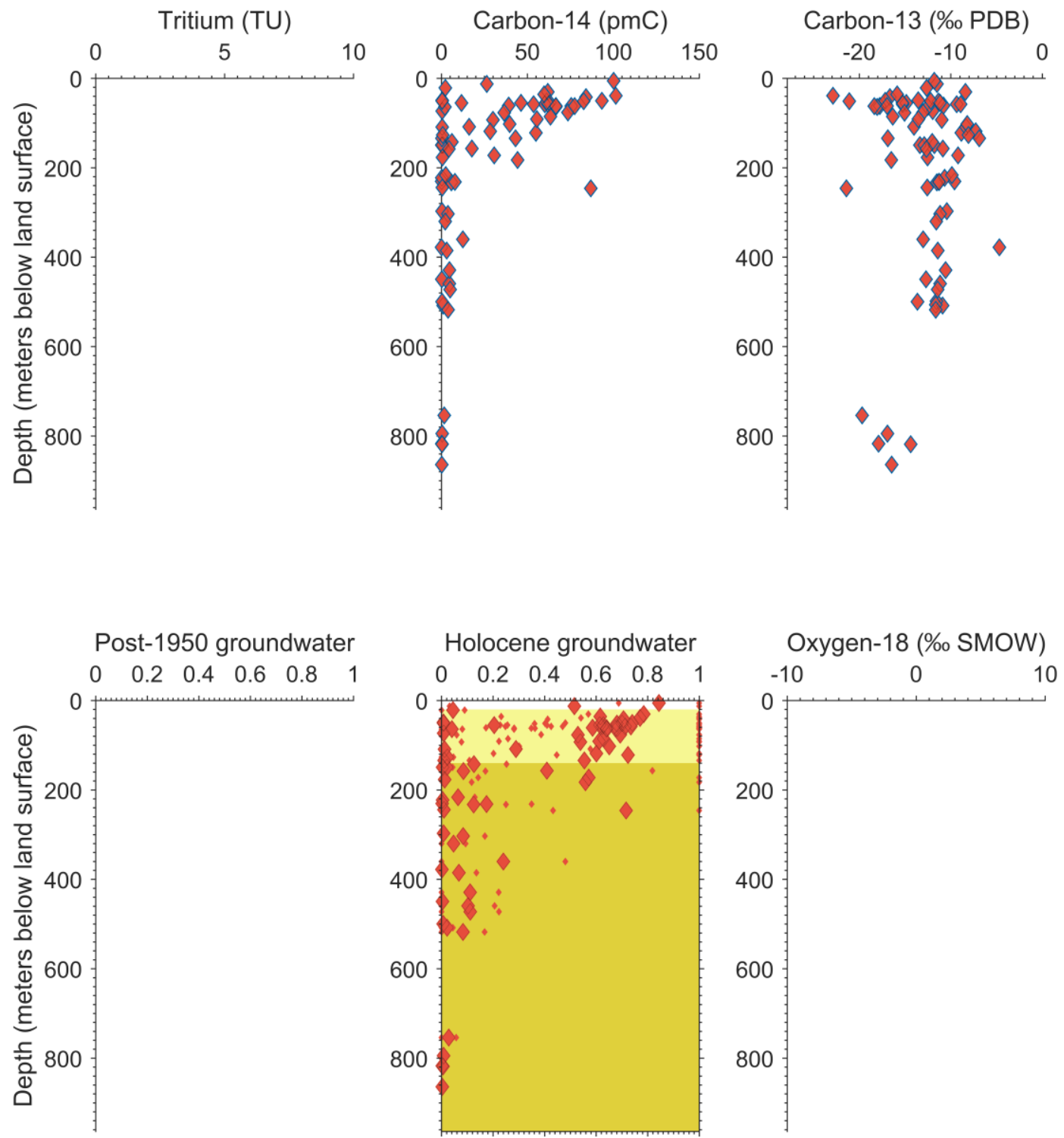

Houston Coastal Lowlands, USA 

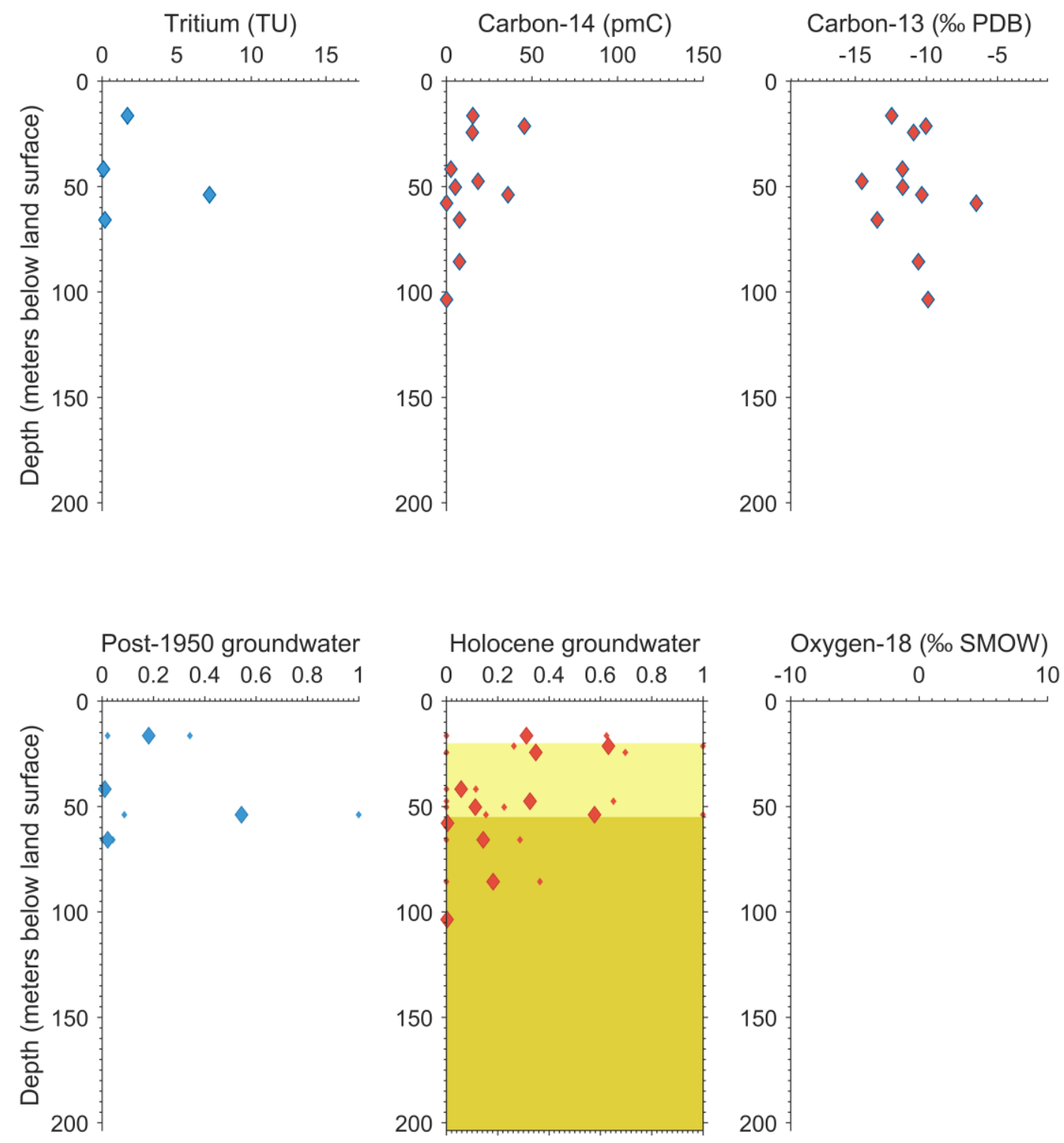

Lower Tertiary Aquifers, USA 

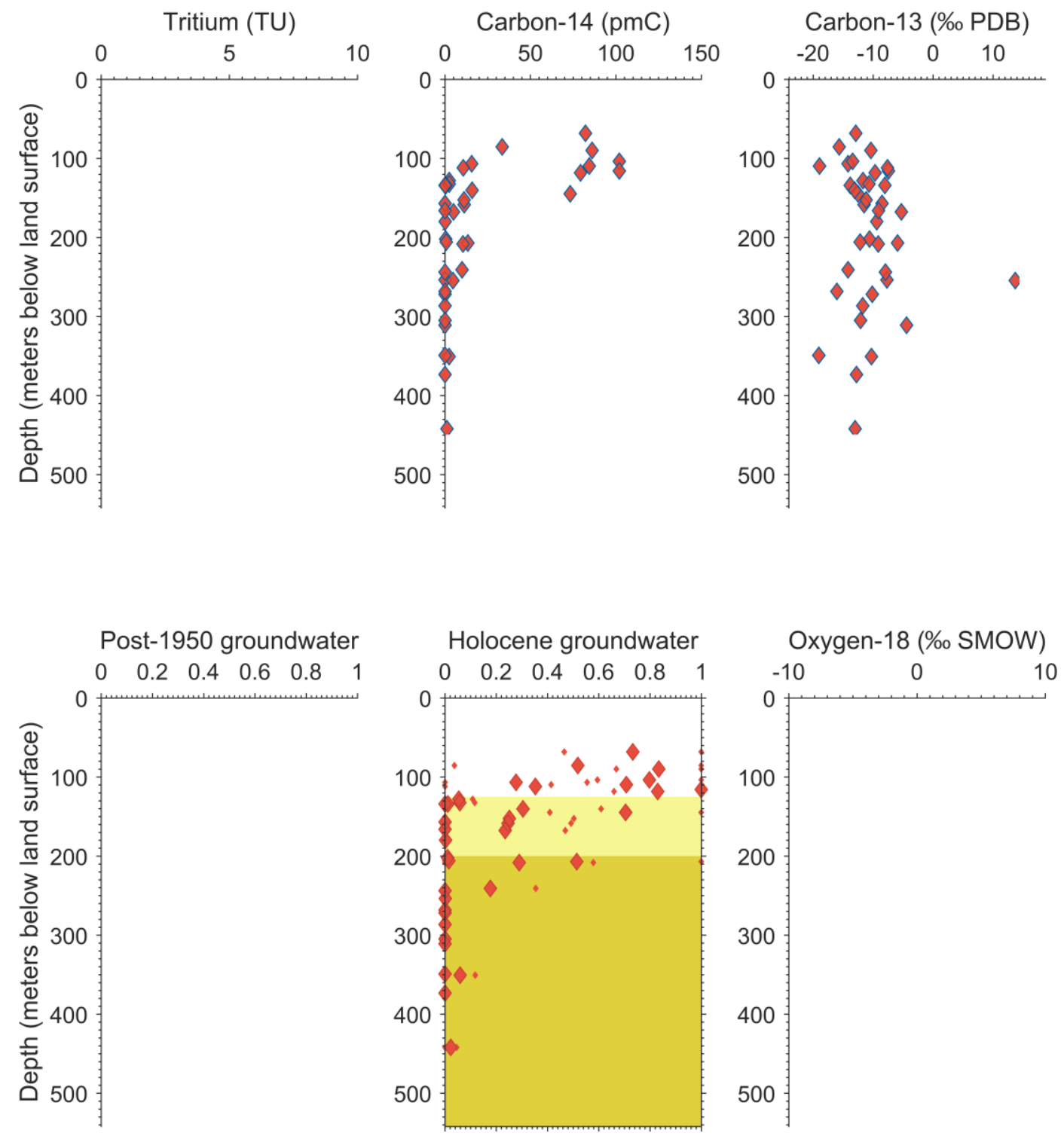

Denver Basin, USA 

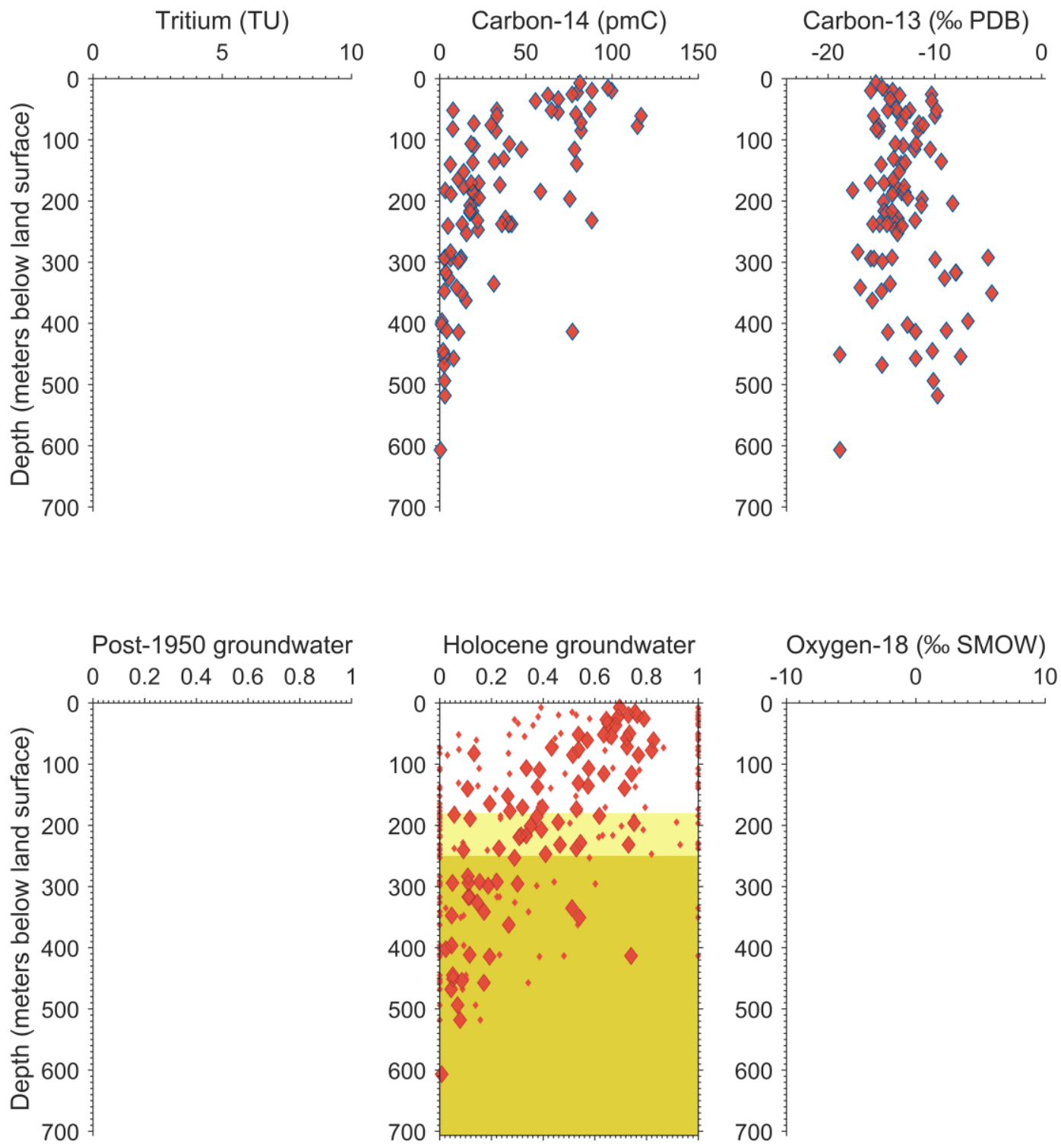

San Diego Basin, USA 

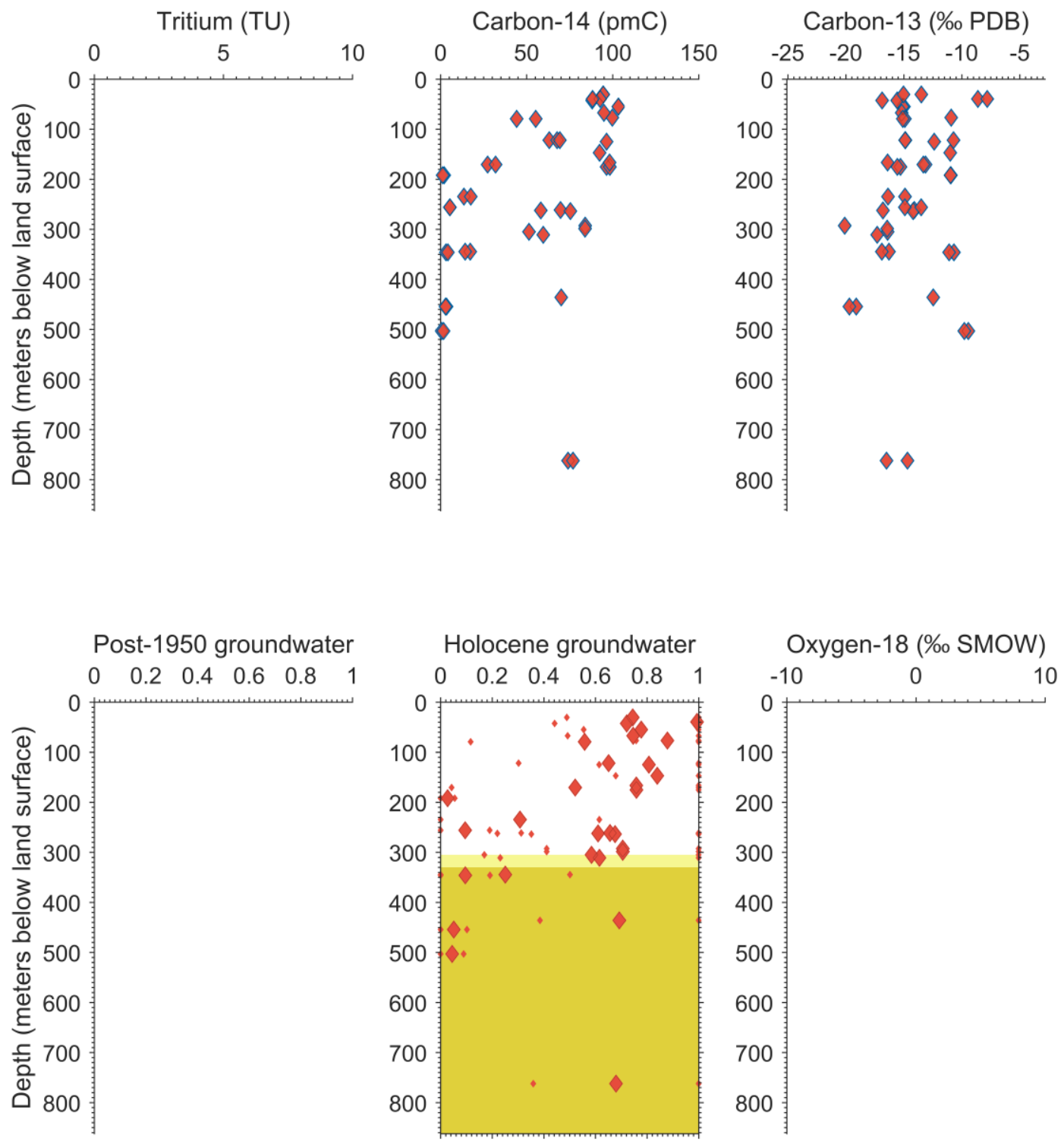

Temecula-Murrieta Basin, USA 

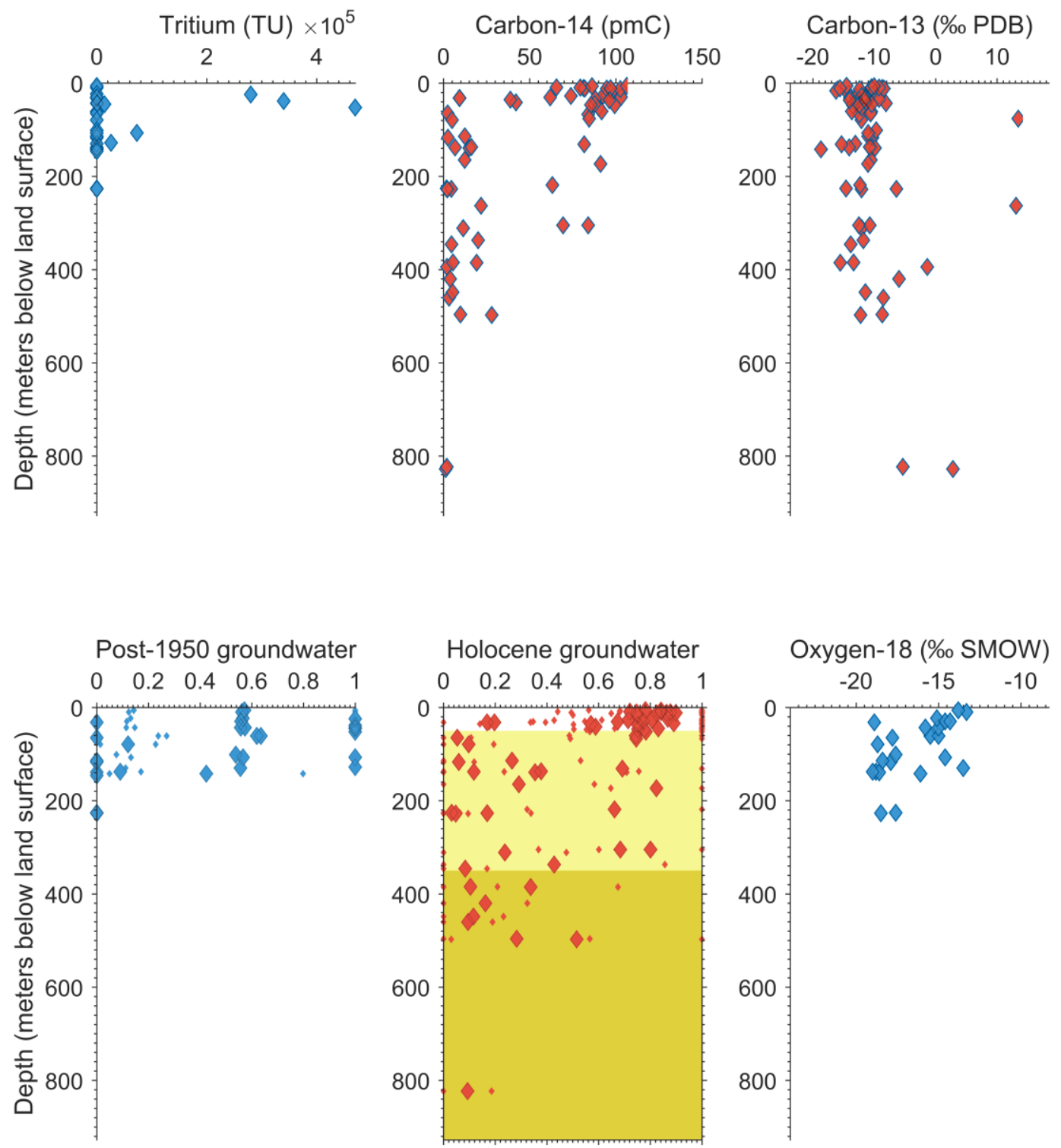

Columbia Basin, USA 

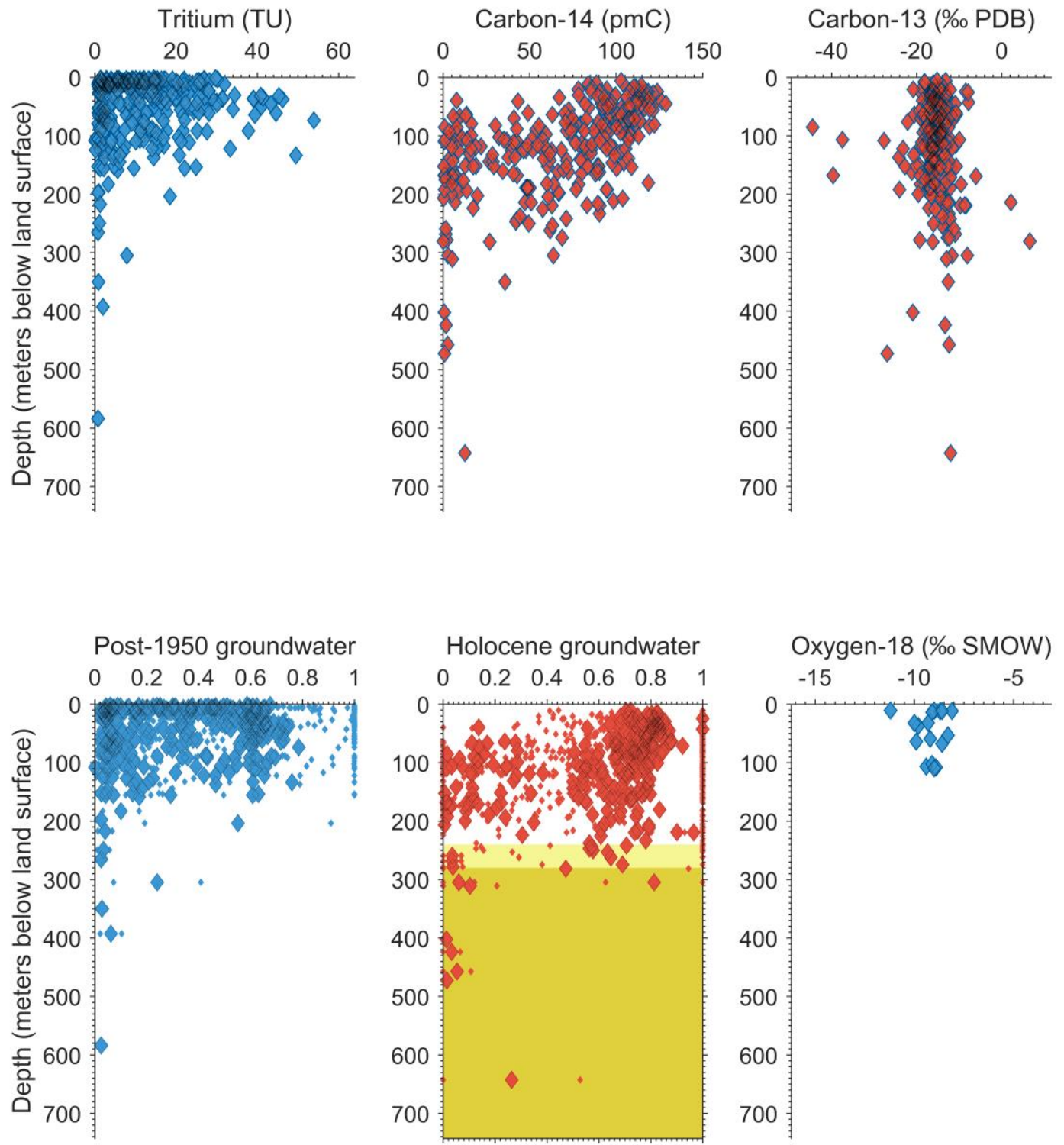

Southern Central Valley, USA 

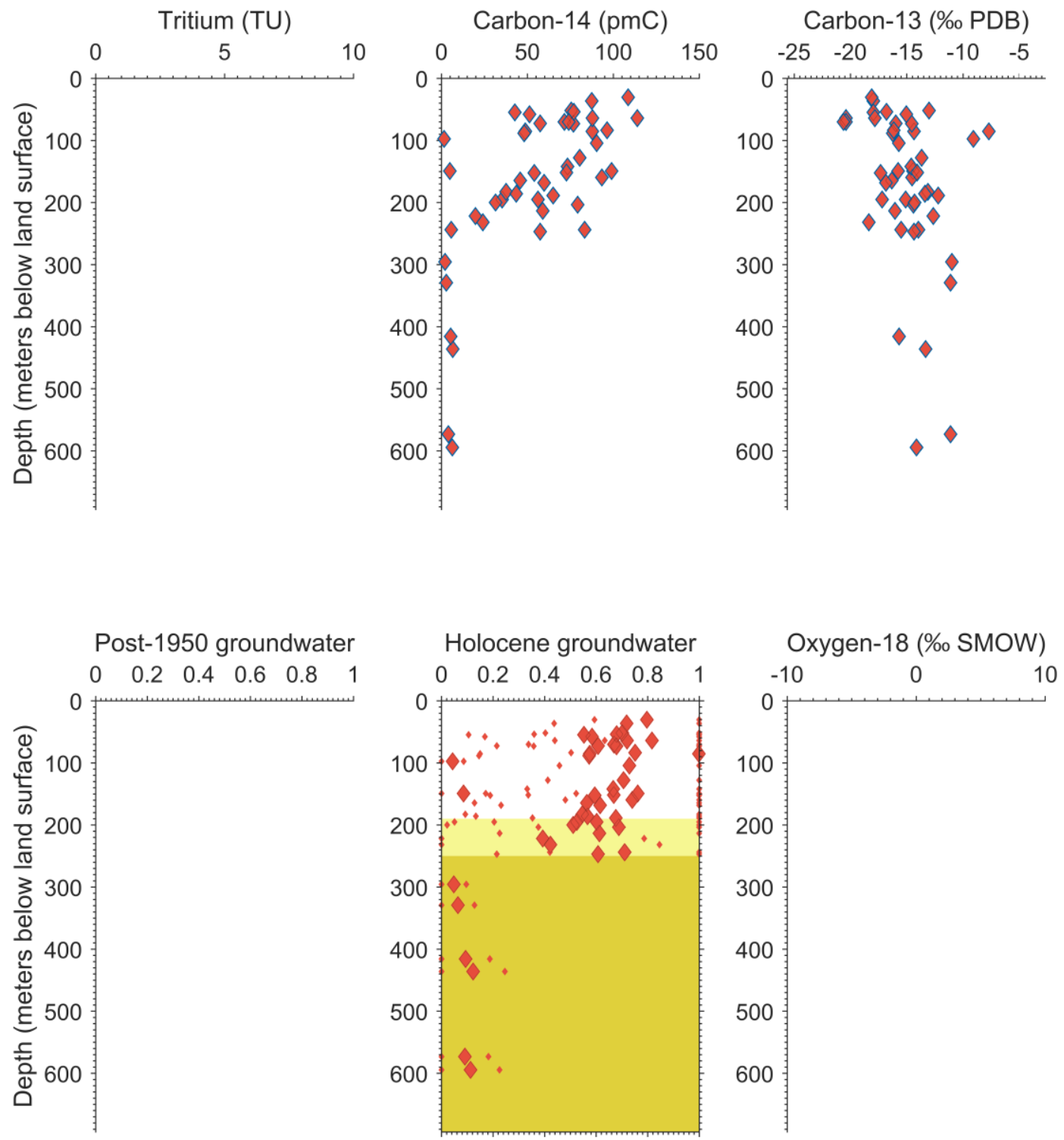

California Bay Area: Monterey Bay, USA 

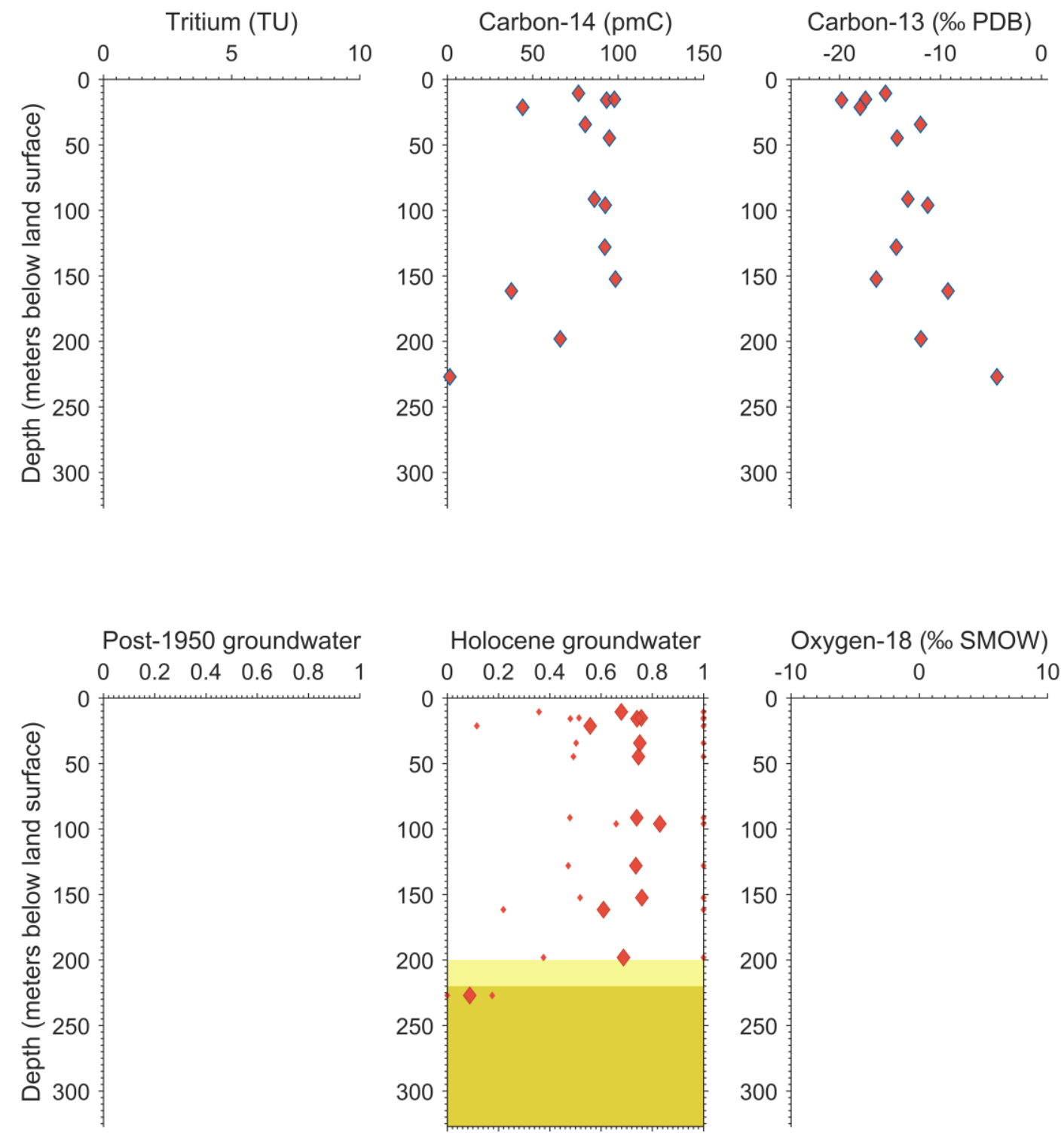

Livermore Valley, USA 

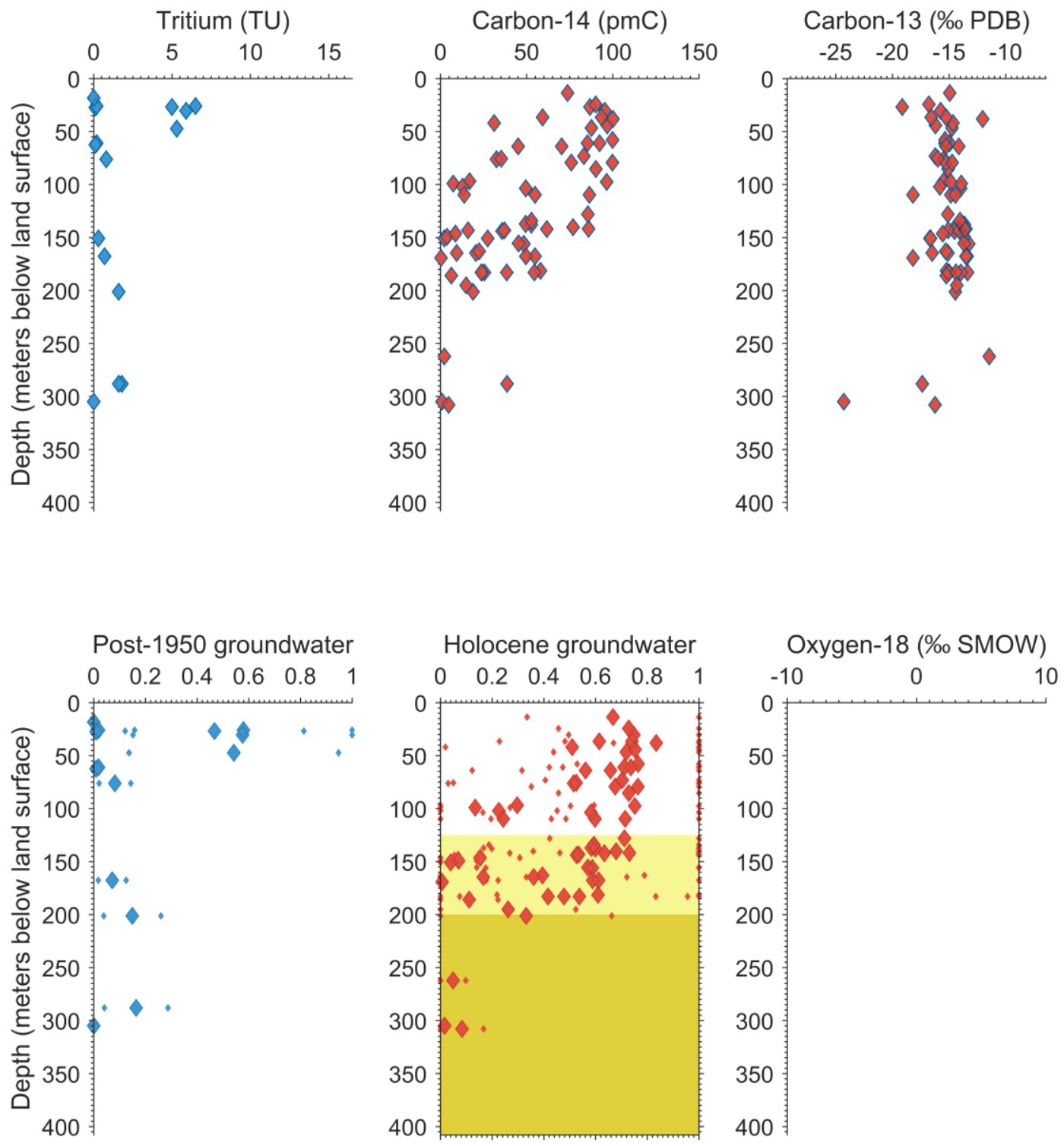

California Bay Area: East, USA 

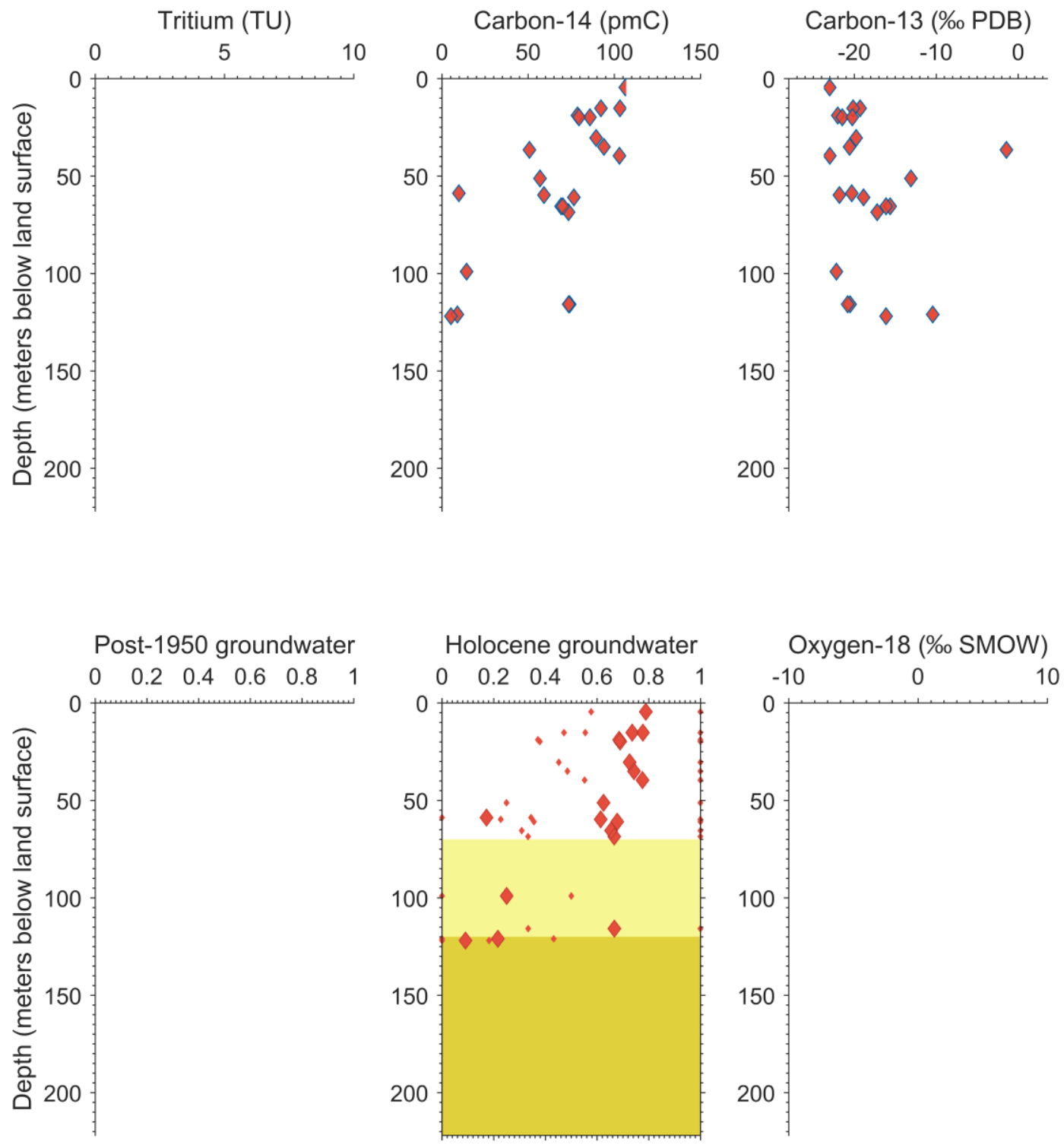

\section{Eureka Coast, USA}



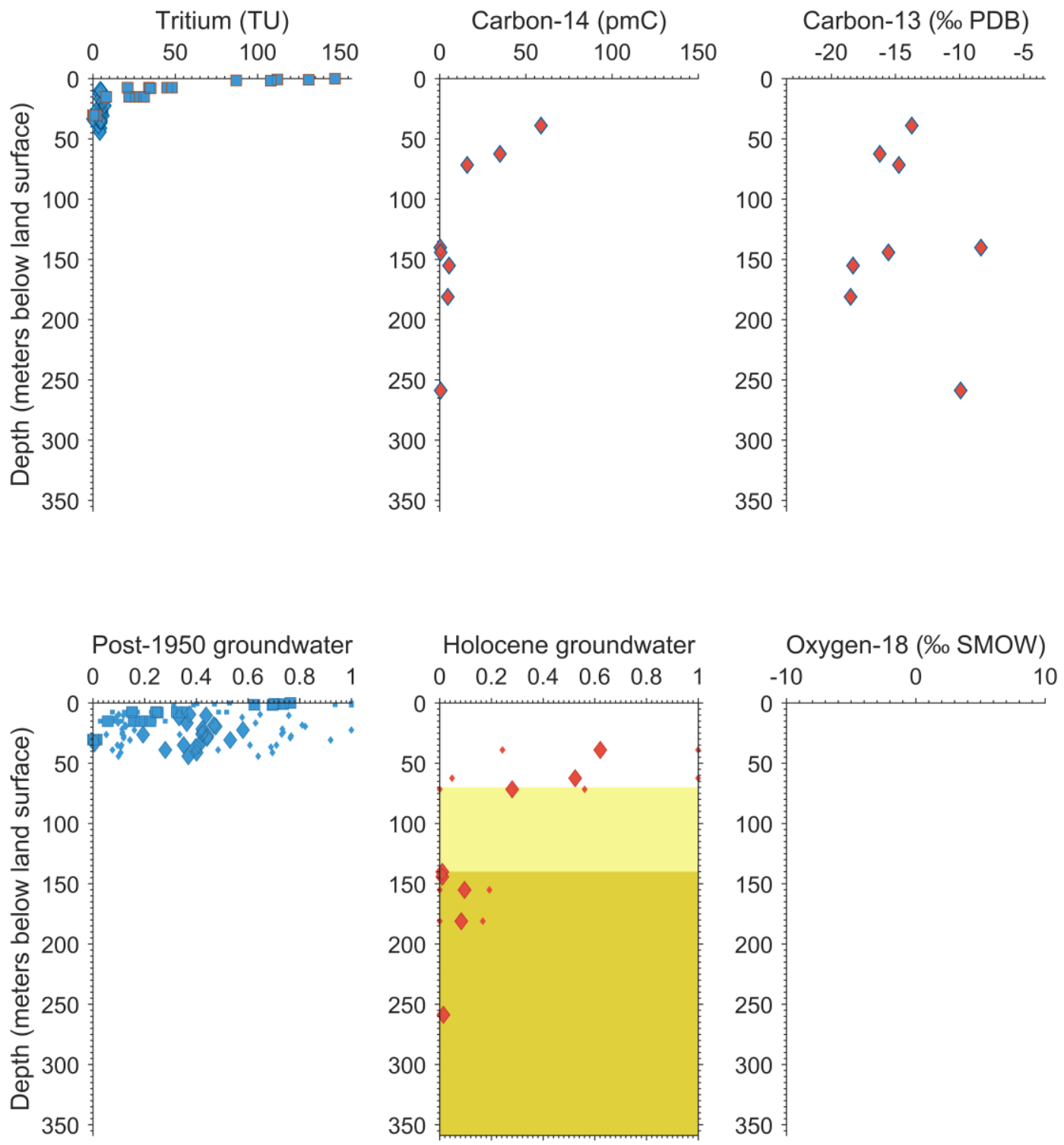

Wharton tract, USA 

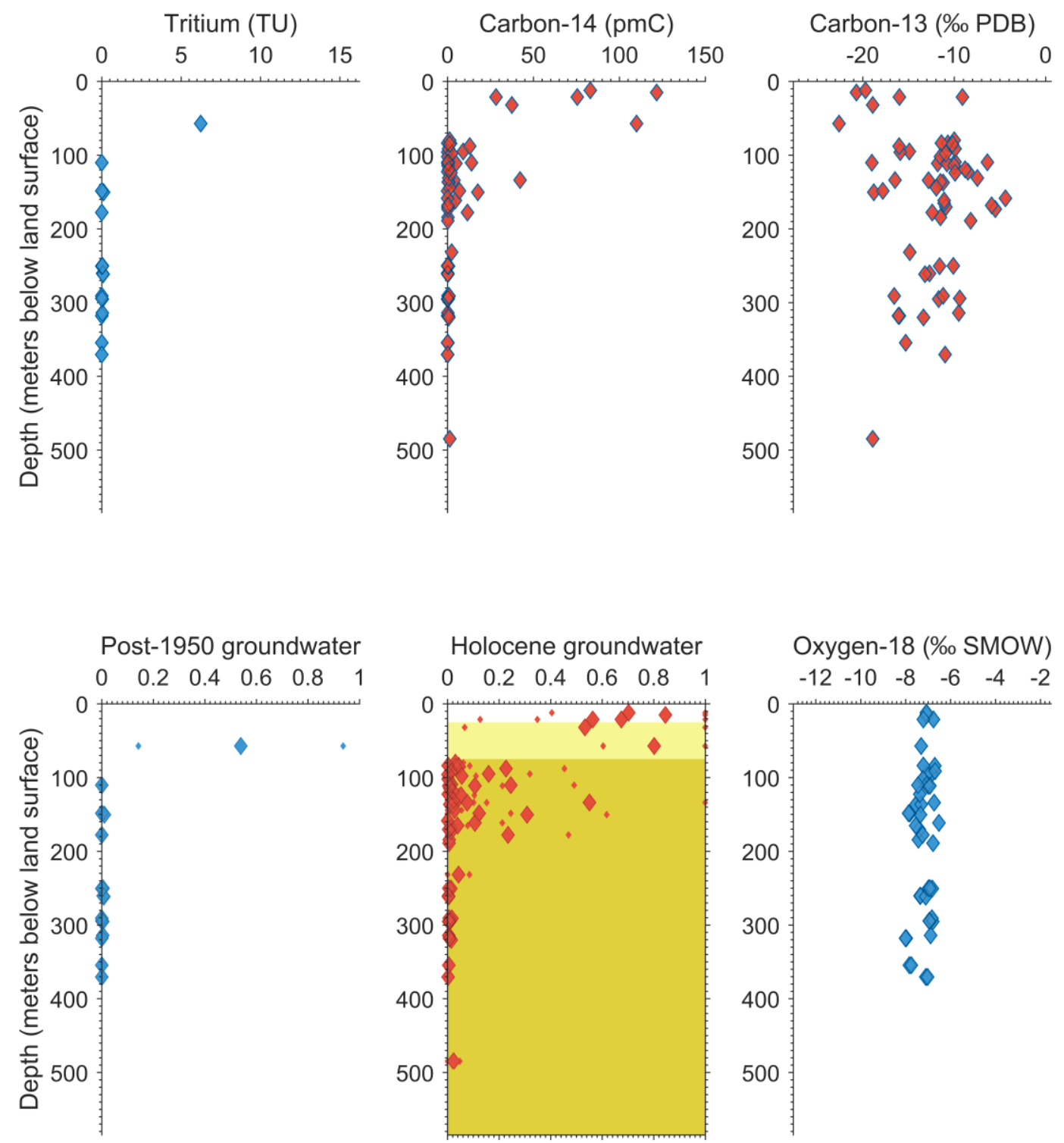

Maryland Coastal Plain (Aquia, Patapsco), USA 

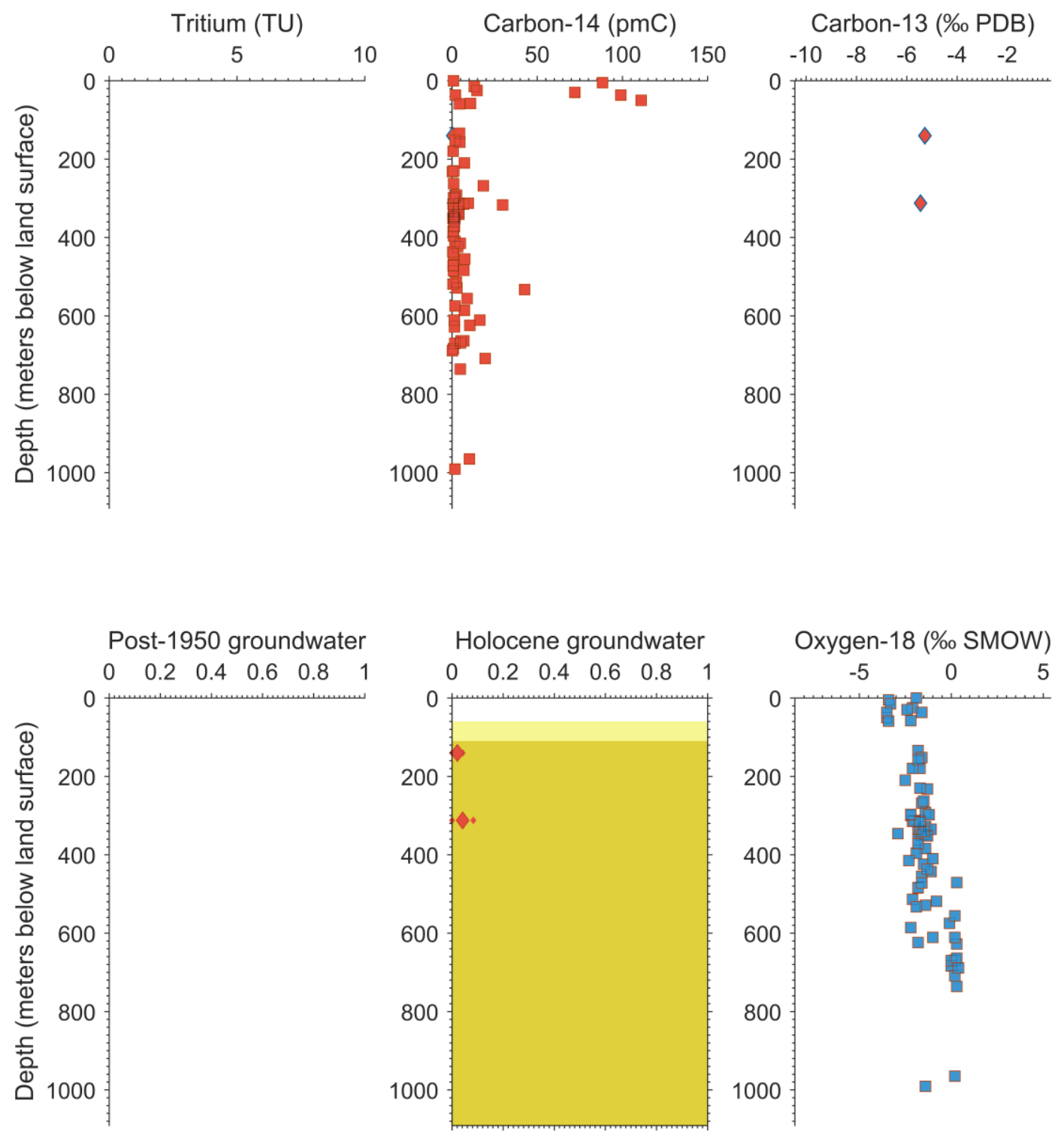

Floridan Surficial Aquifers, USA (Paleowater transition based on oxygen-18) 

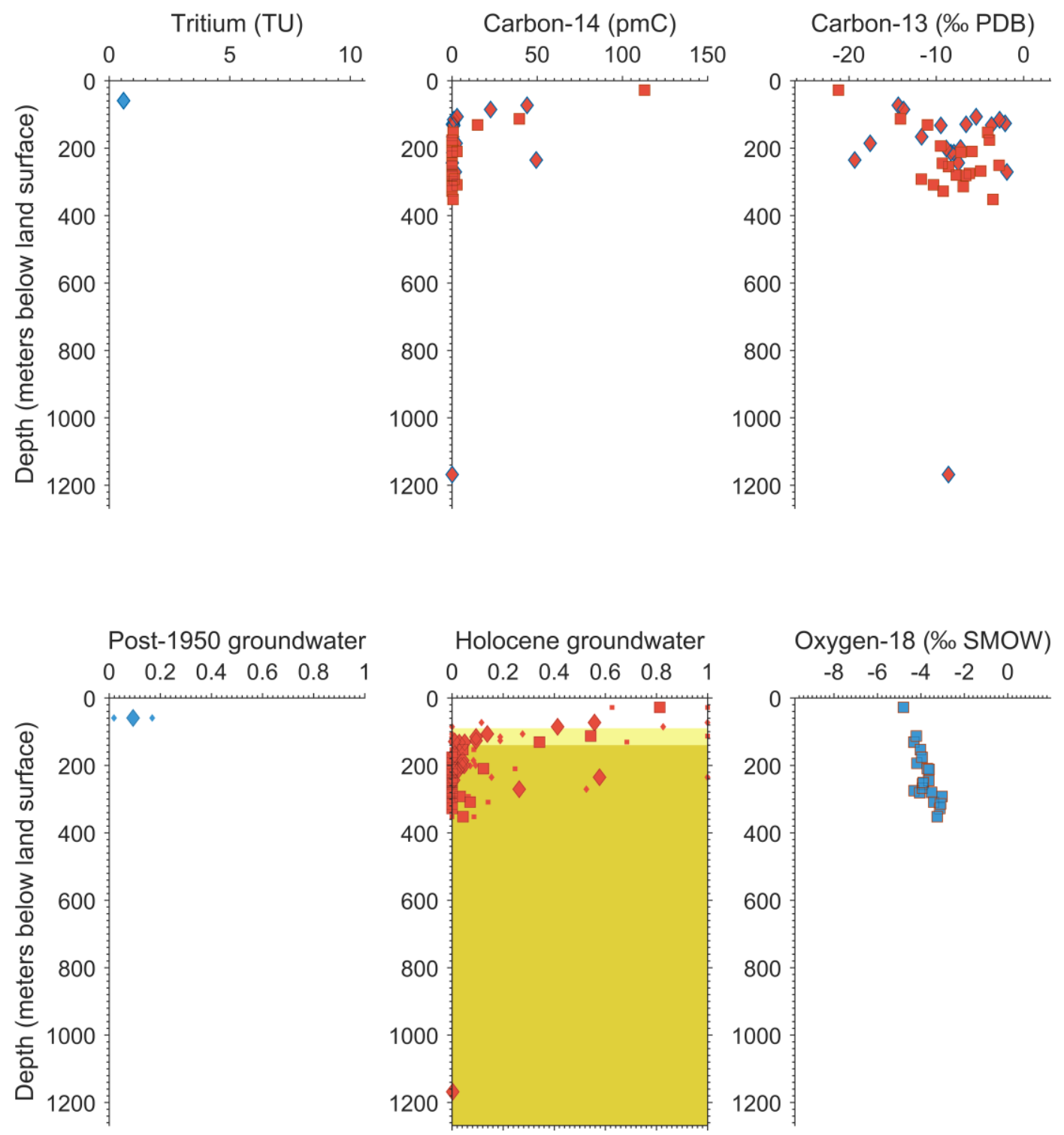

Floridan Aquifer System, USA 

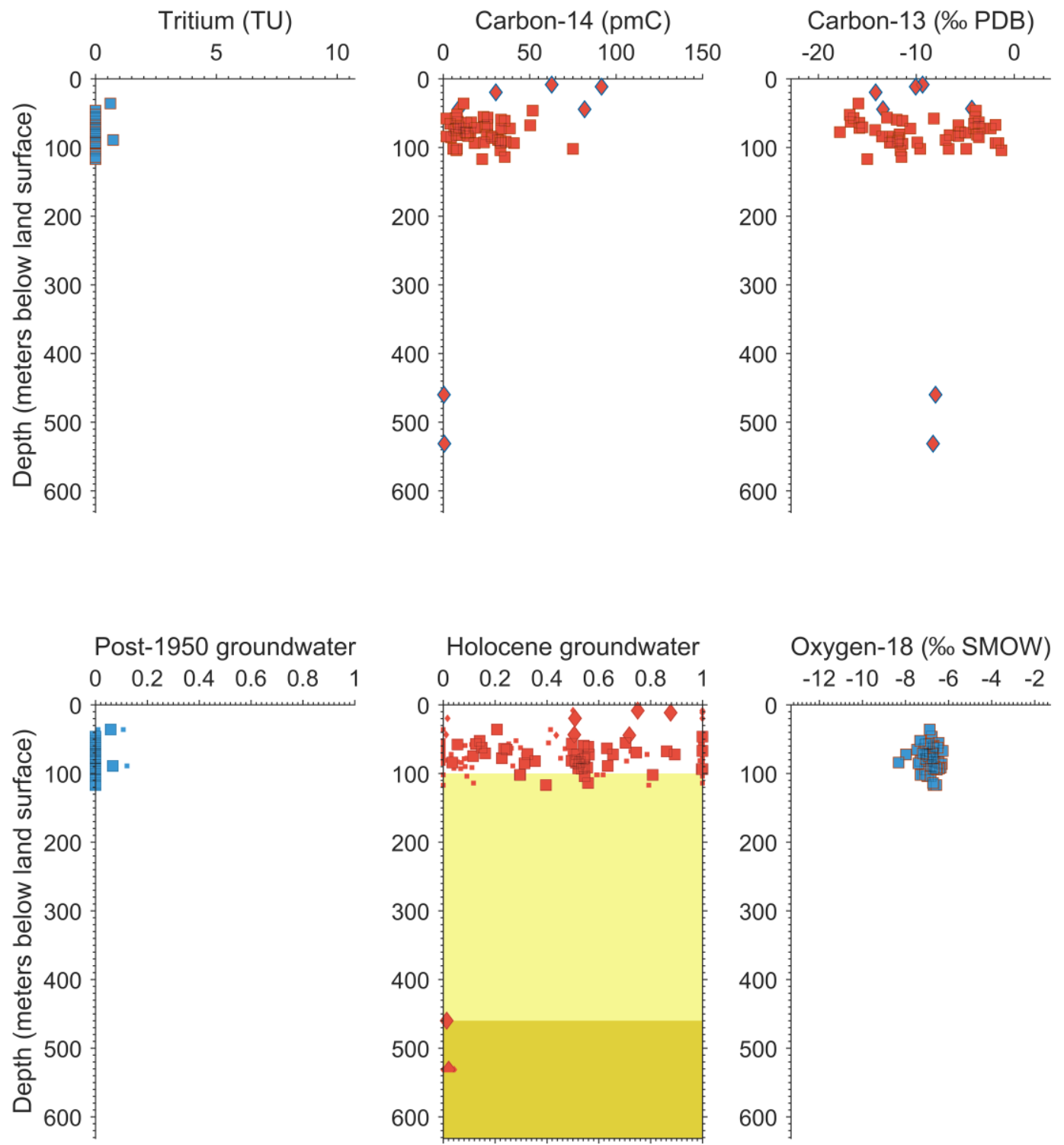

Mahomet Aquifer, USA 

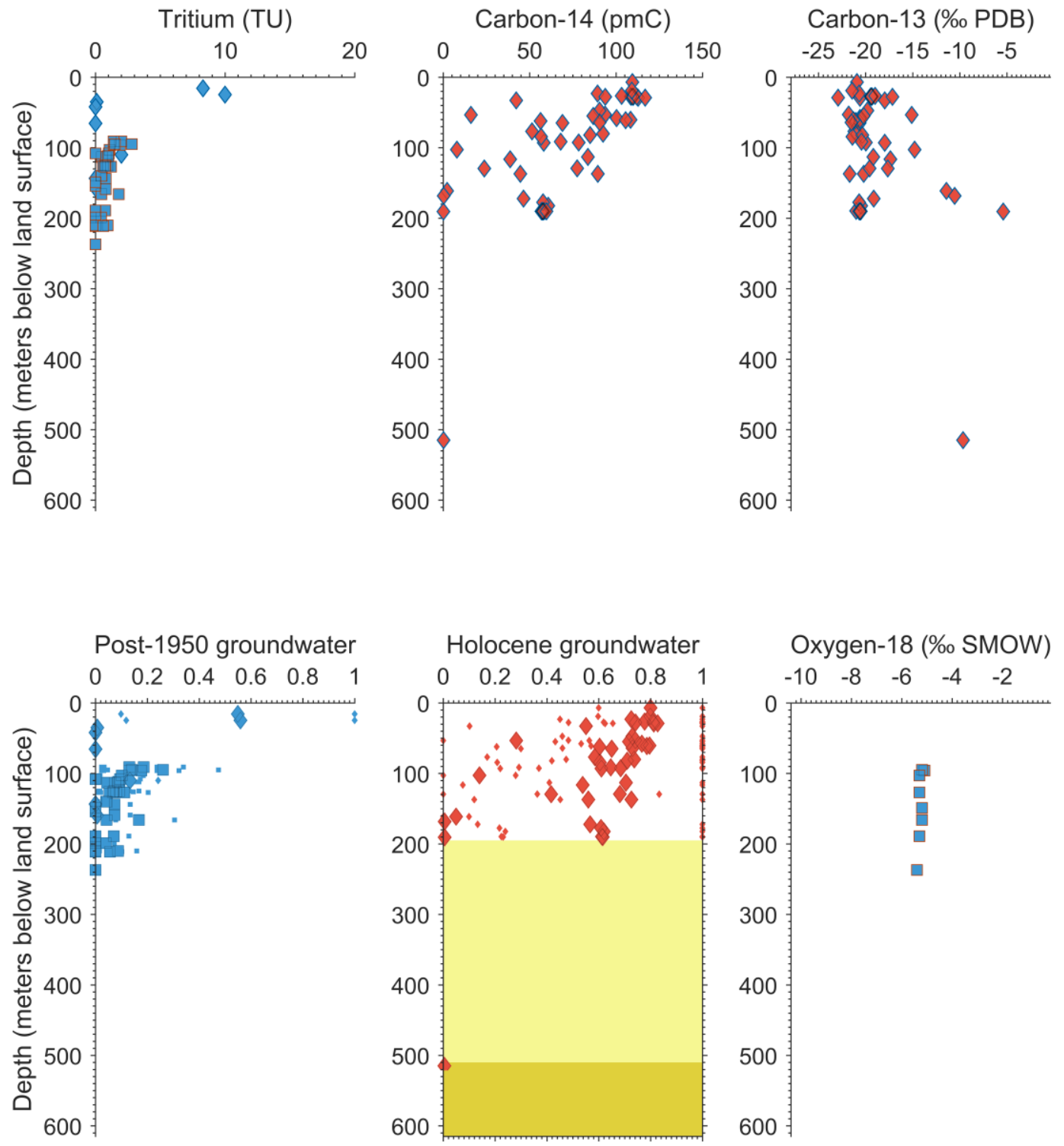

Mississippi embayment: Memphis aquifer, USA 

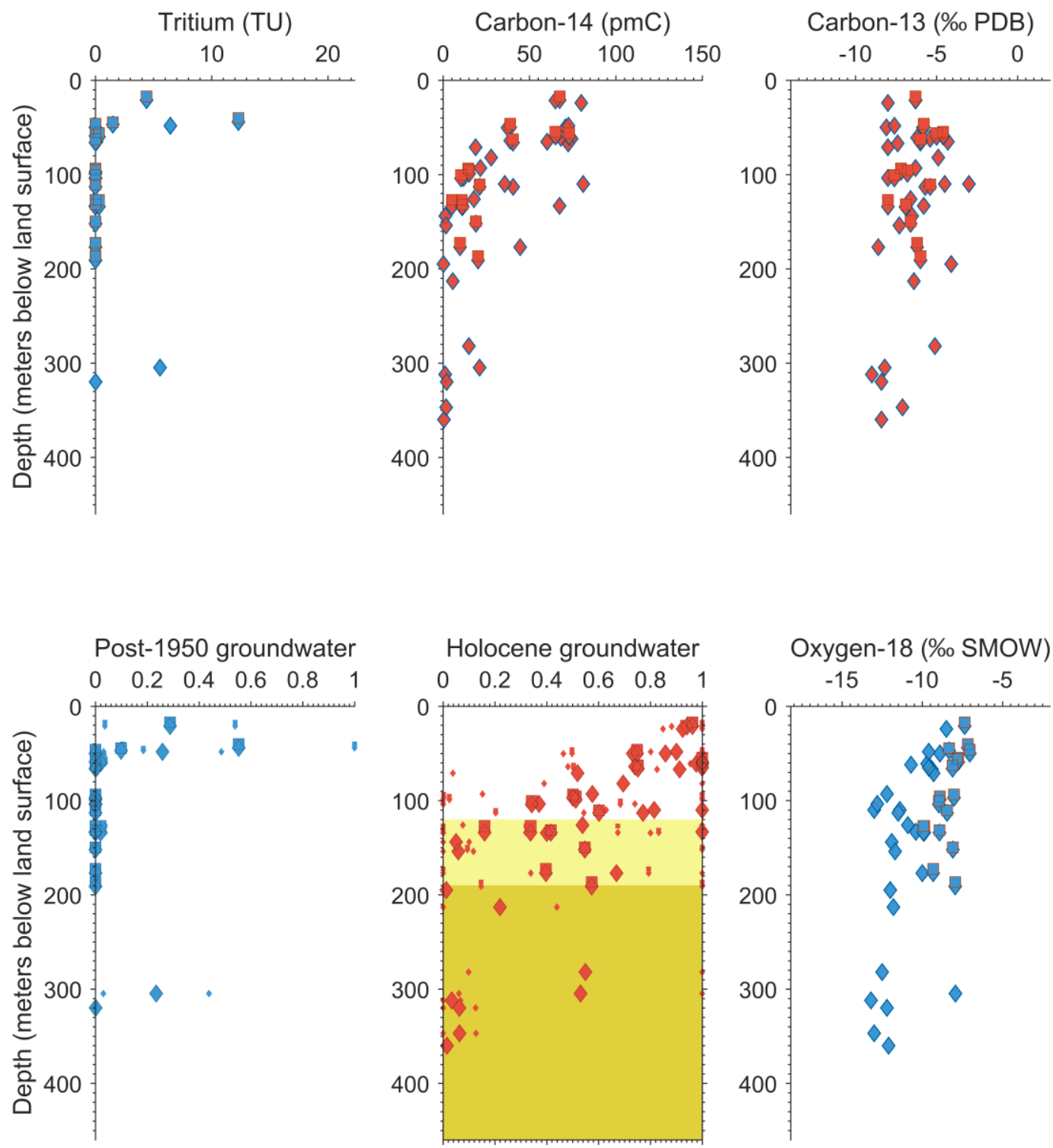

High Plains Aquifer: Central (Ogallala), USA 

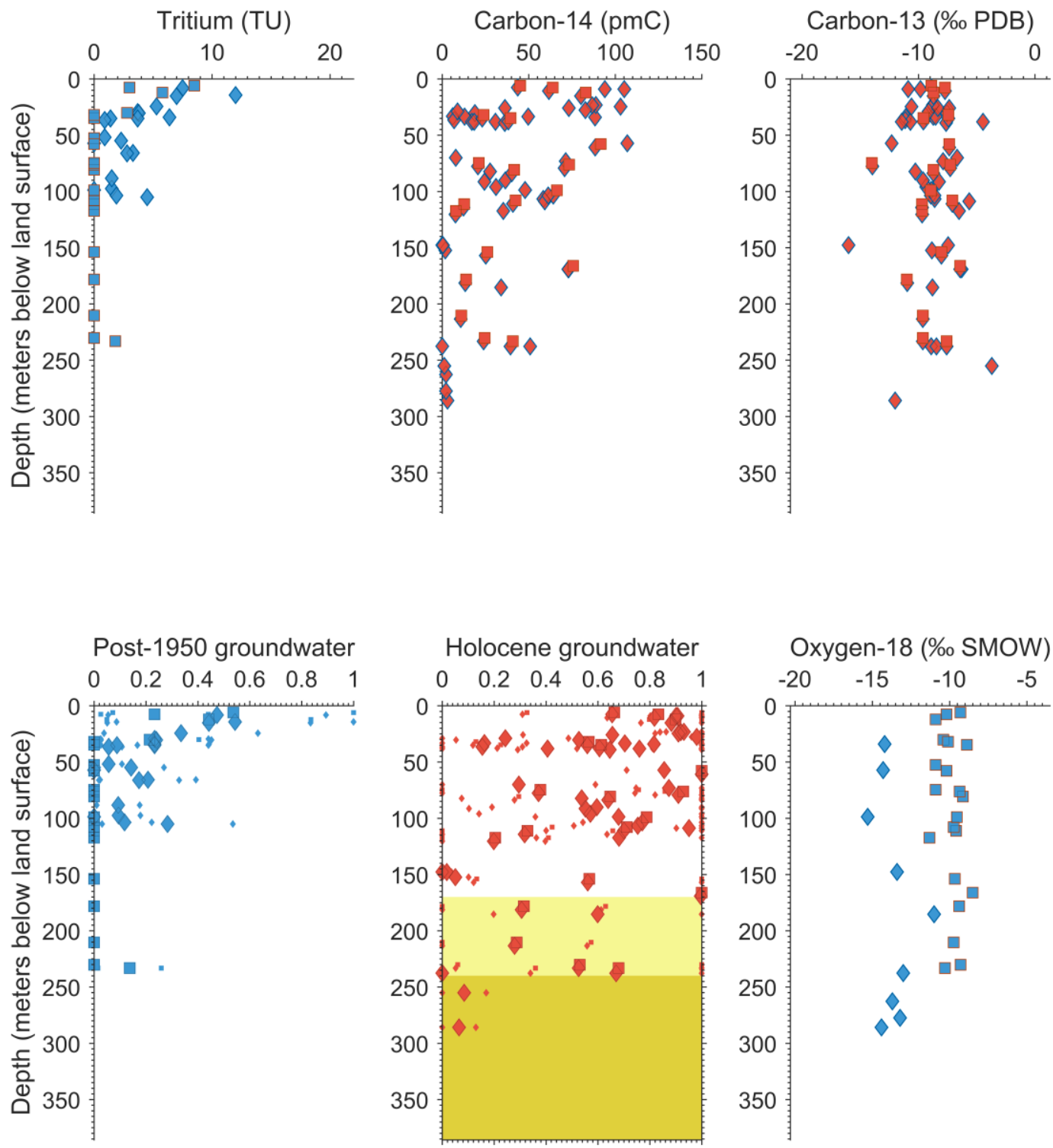

High Plains Aquifer: North (Ogallala), USA 

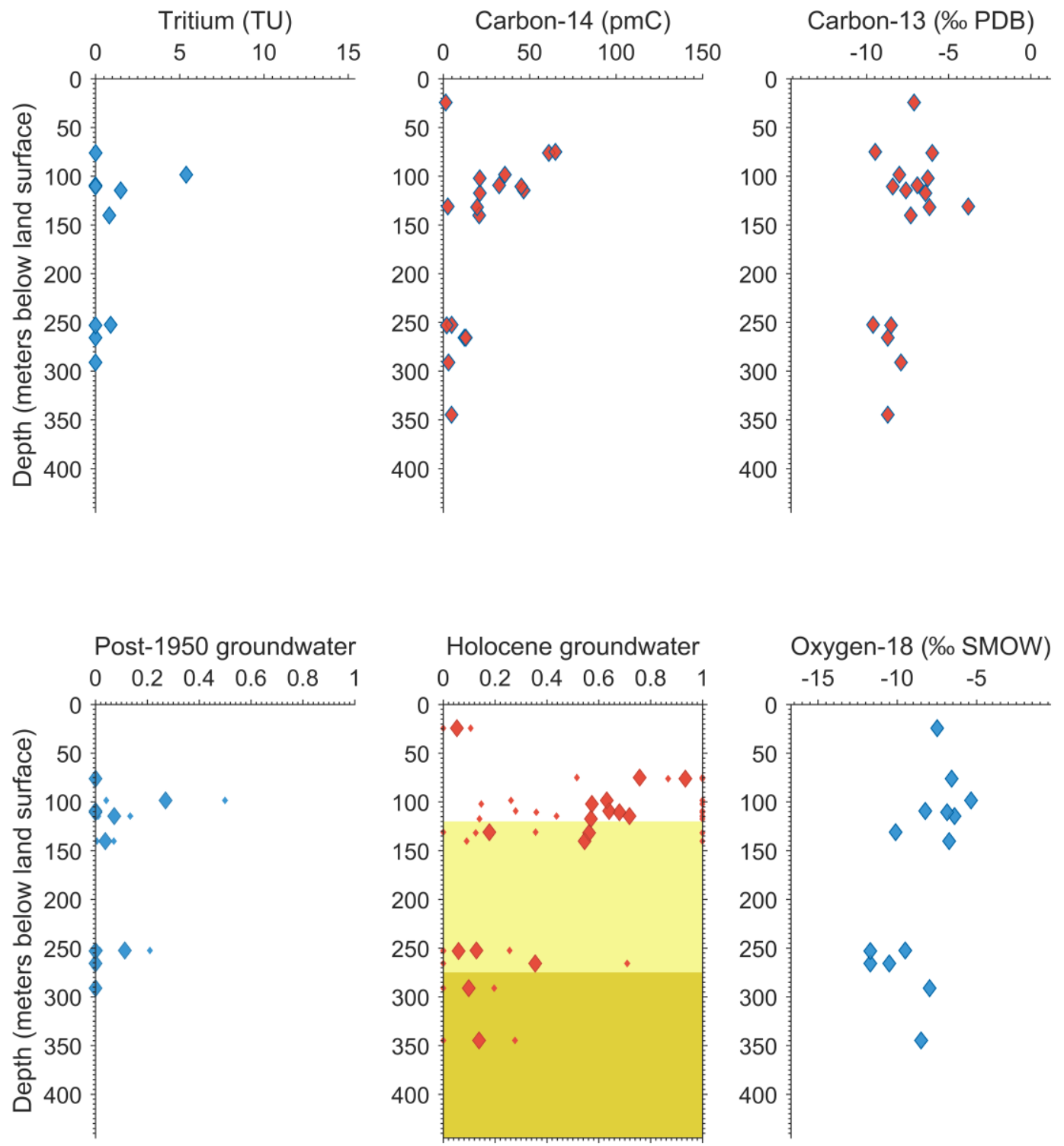

High Plains Aquifer: South (Dockum), USA 

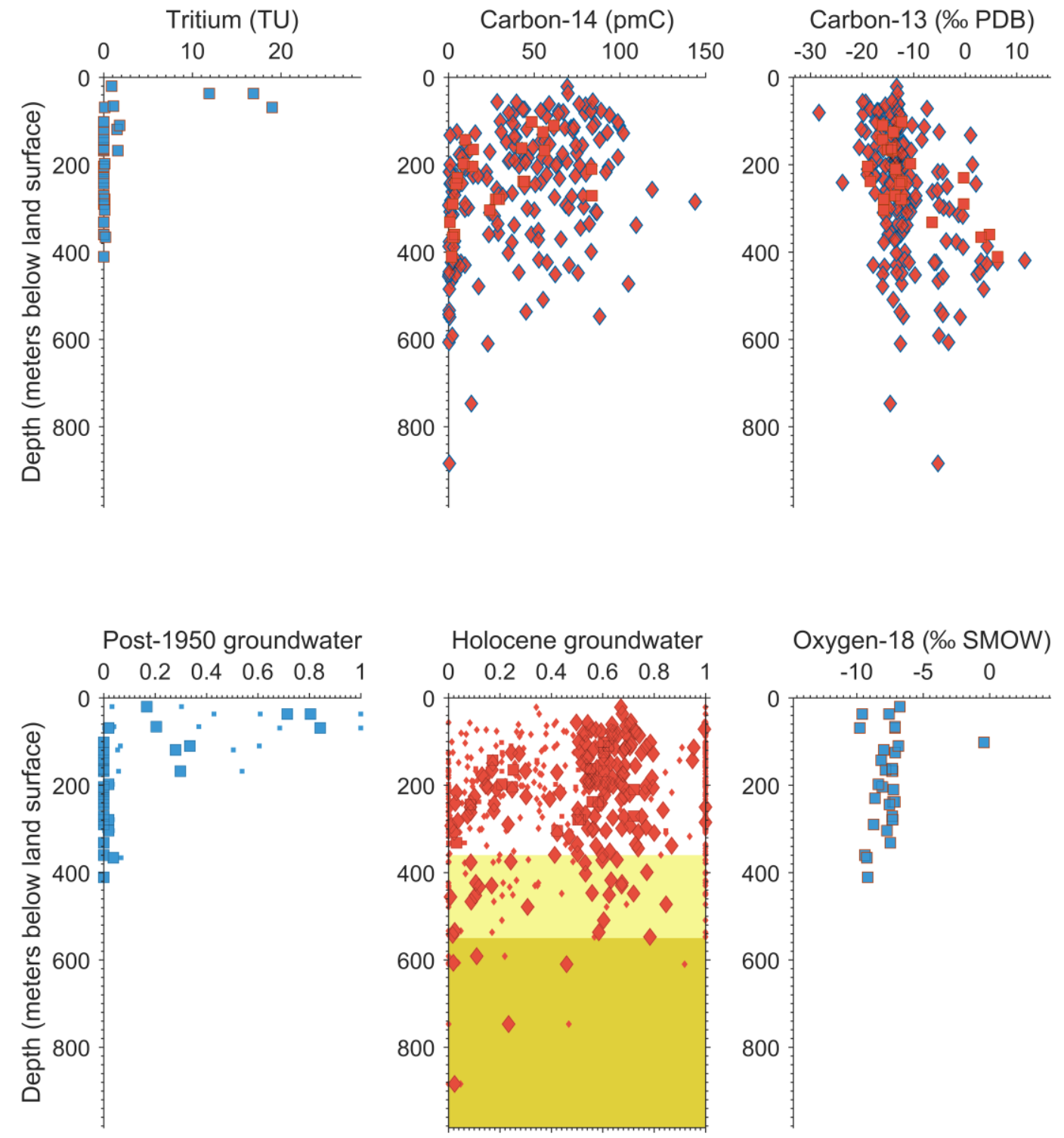

Los Angeles Basin, USA 

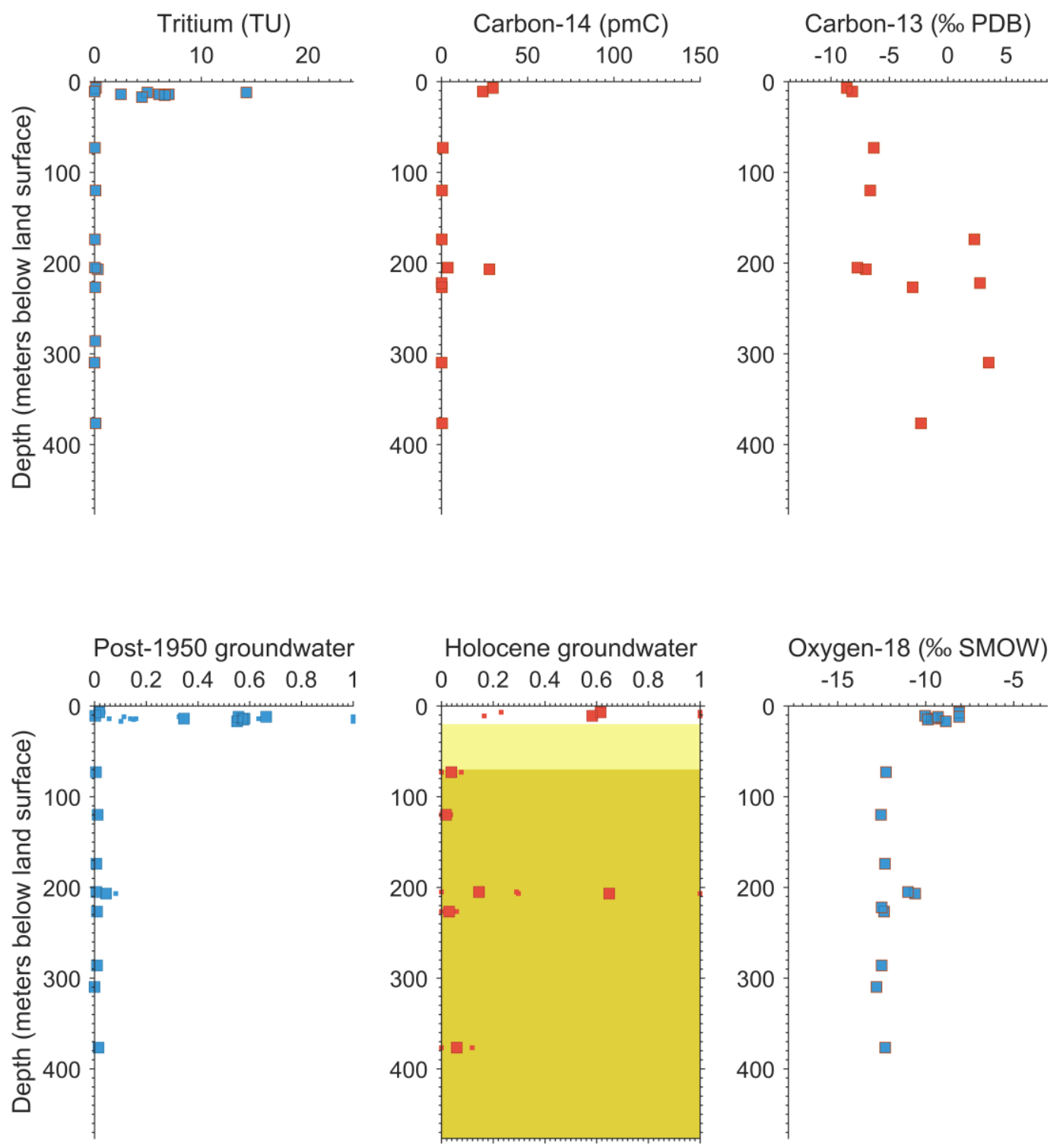

Kazan Trona Ore Field, Turkey (Paleowater transition based on oxygen-18) 

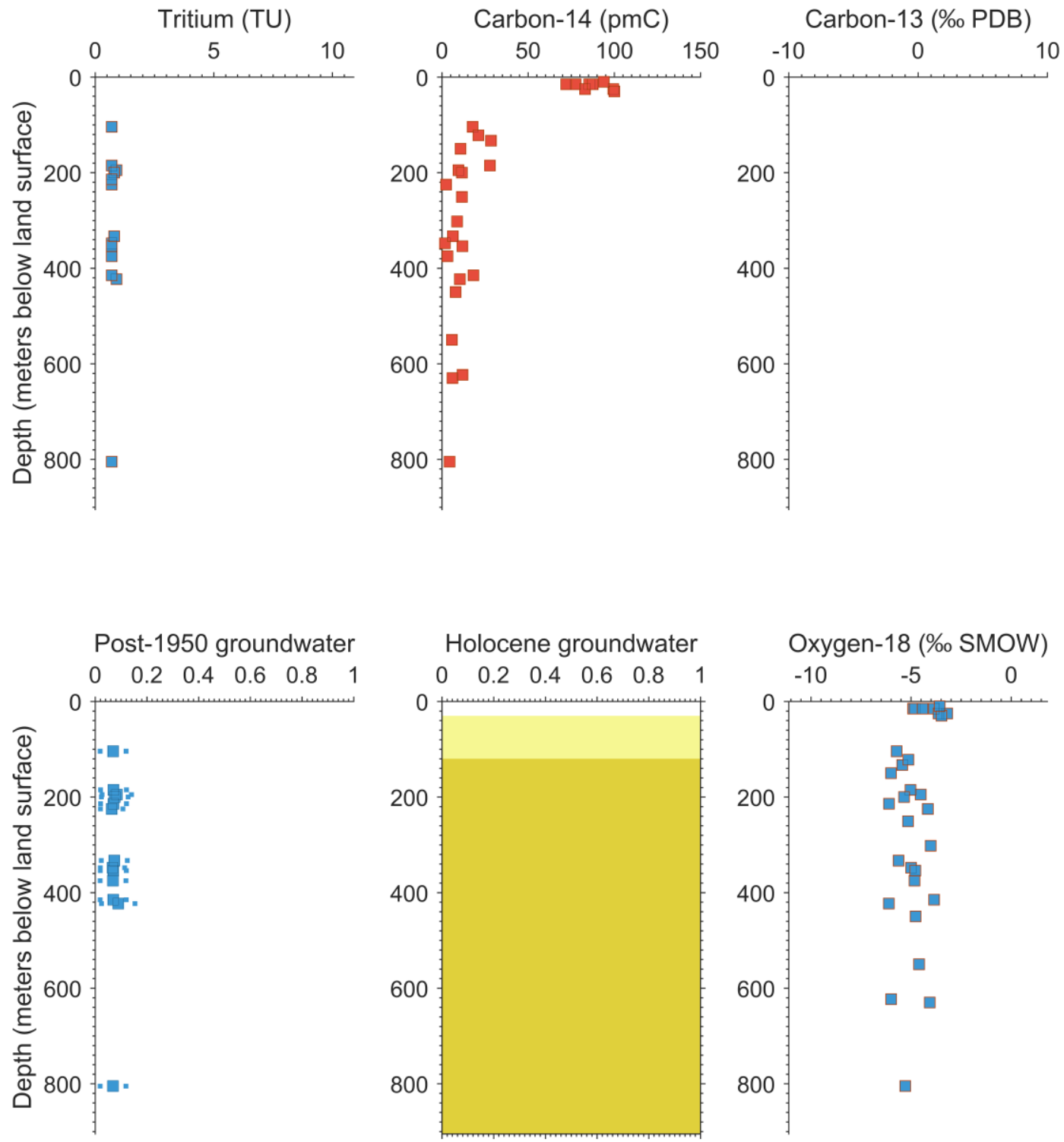

Le bassin du Djérid, Tunisia (Paleowater transition based on oxygen-1i 

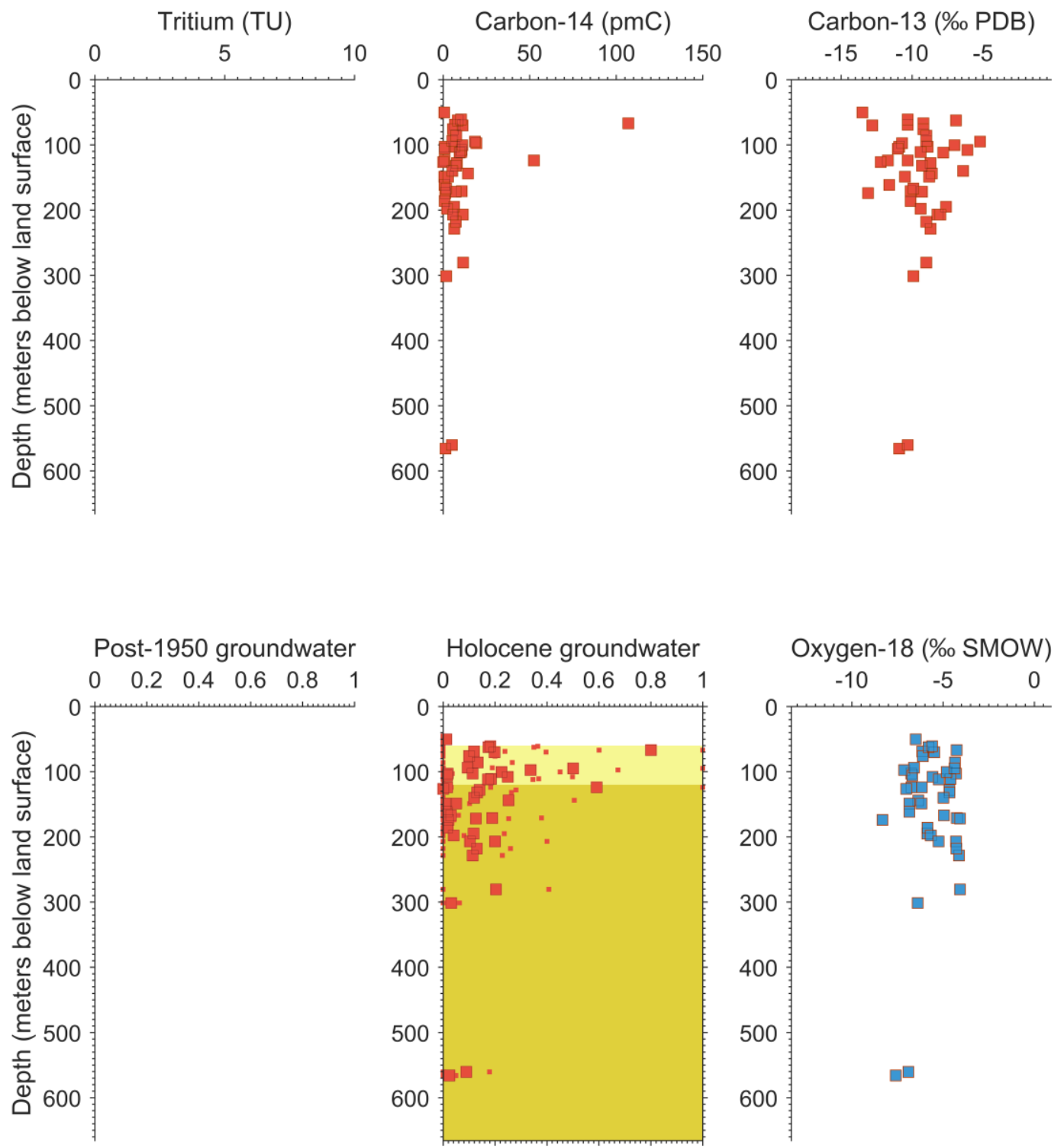

Bangkok Basin, Thailand 

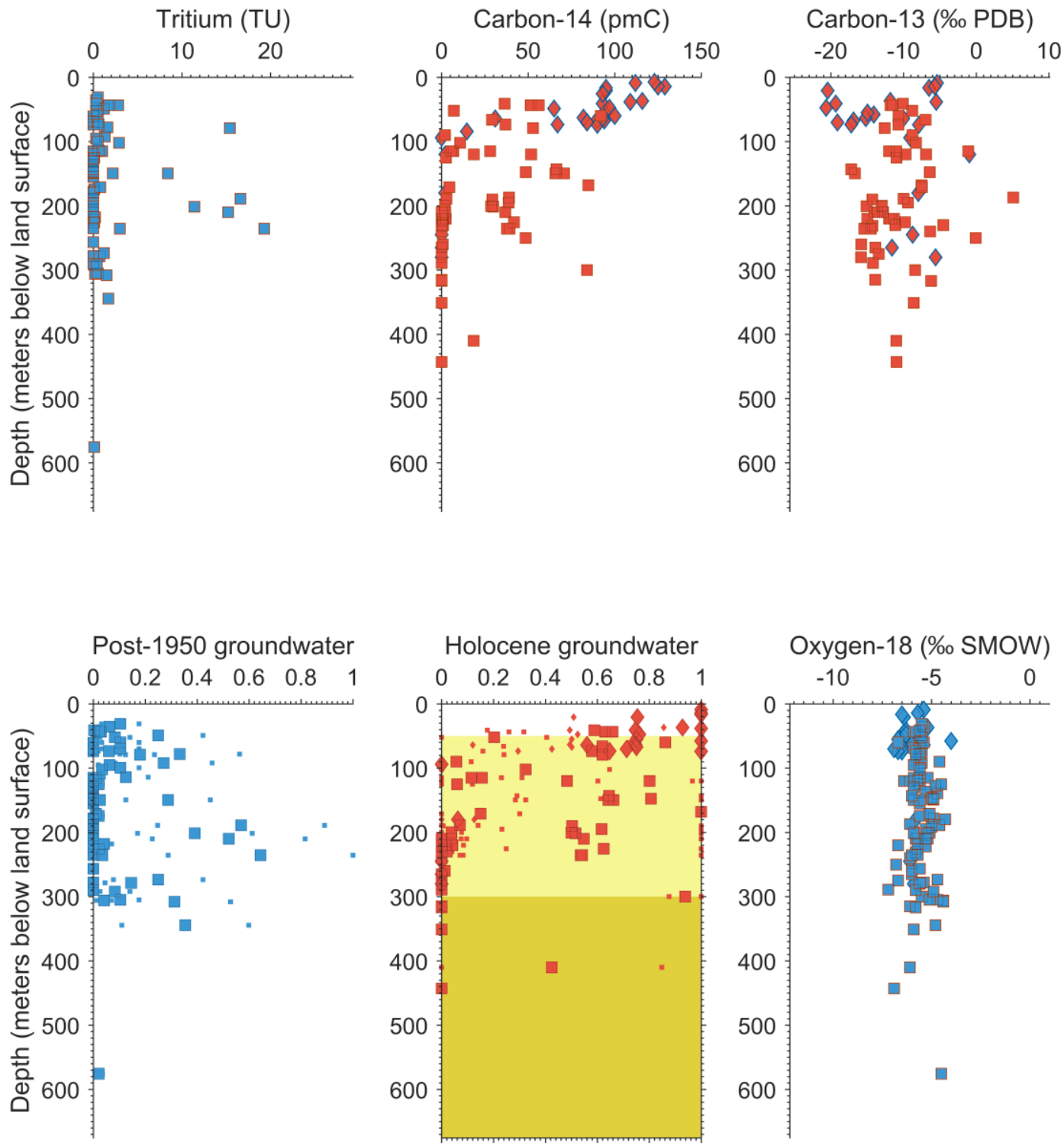

Diass aquifer system, Senegal 

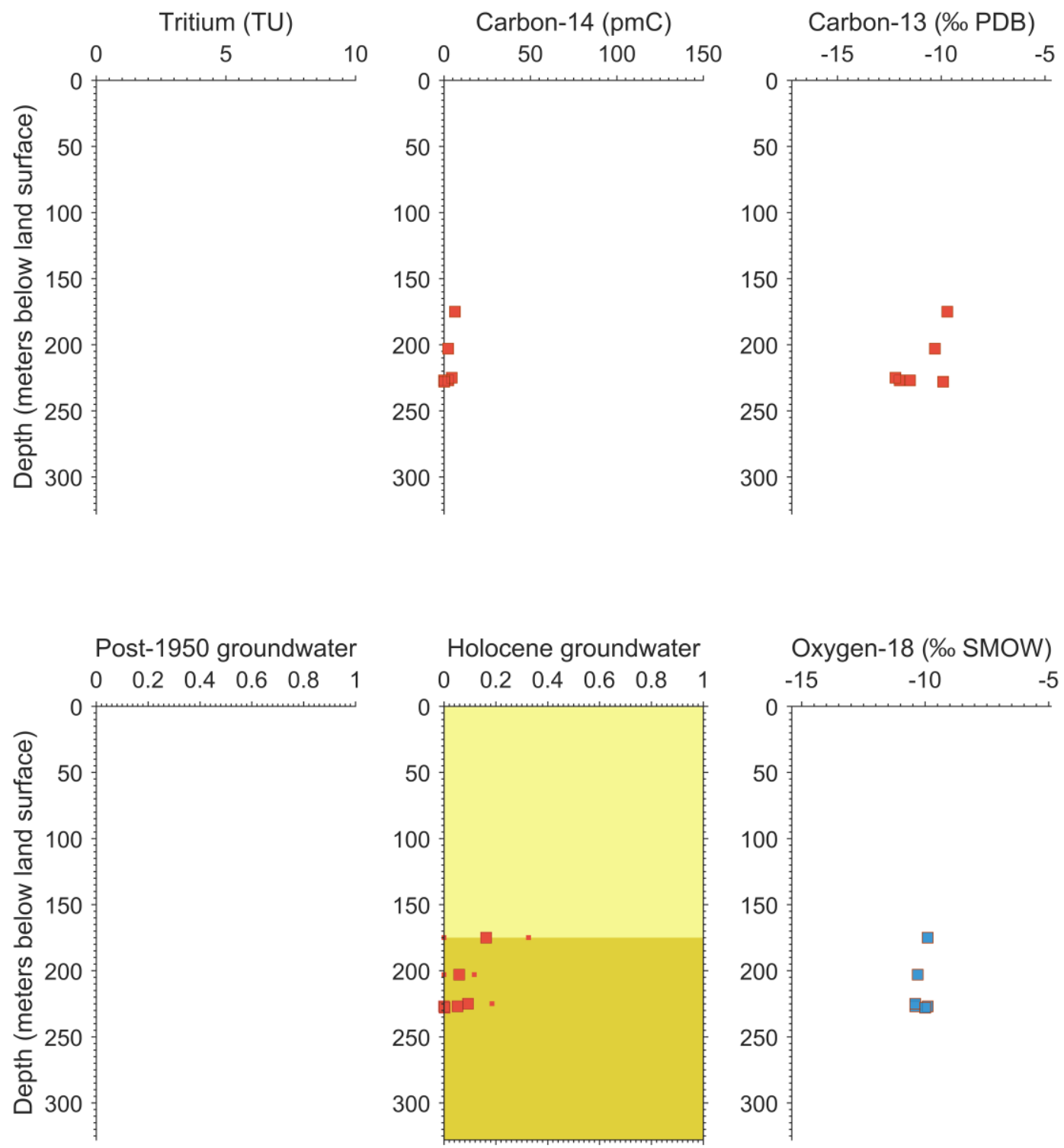

Mazovian basin: Oligocene aquifer, Poland 

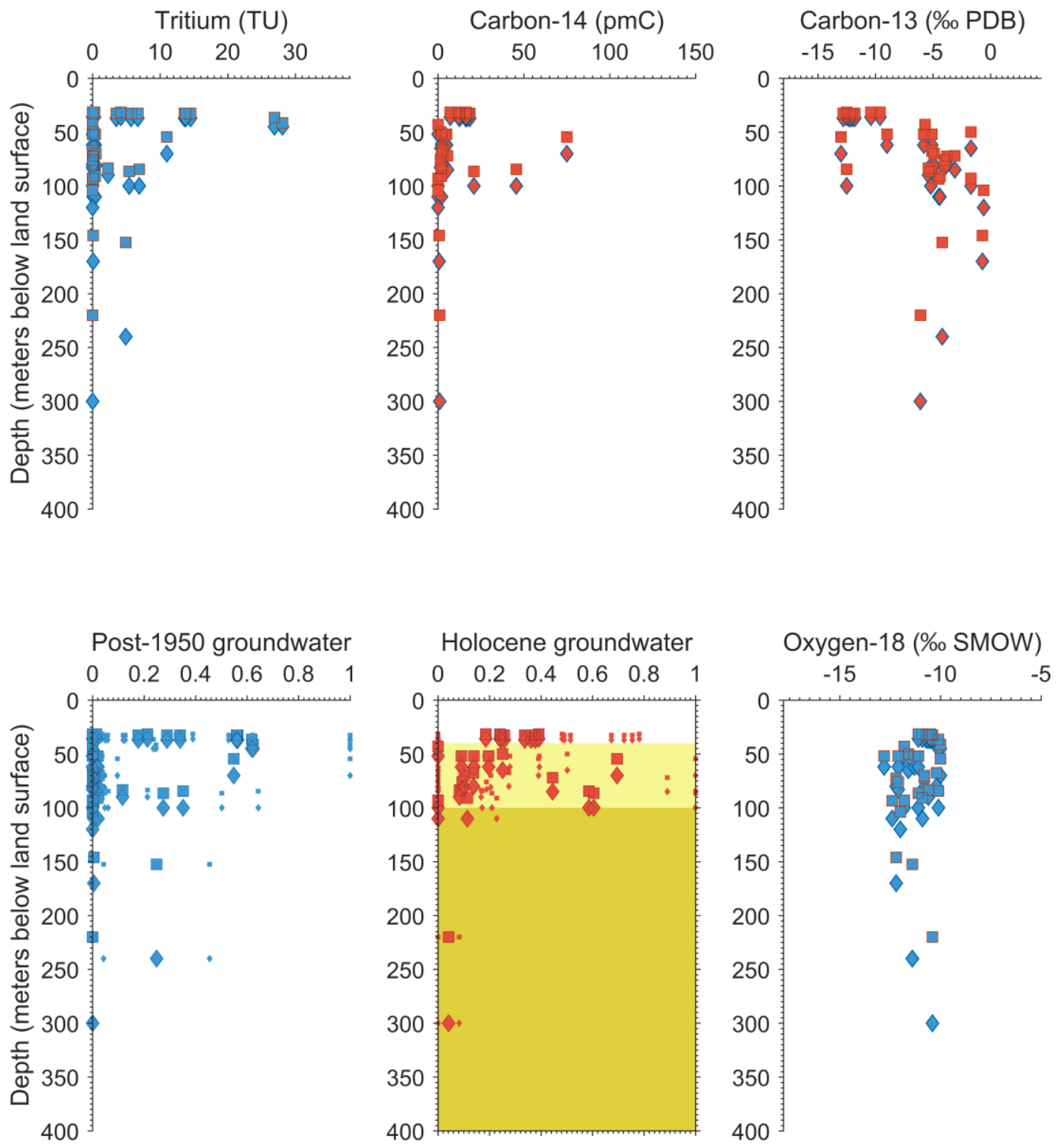

Malm Limestones, Poland 

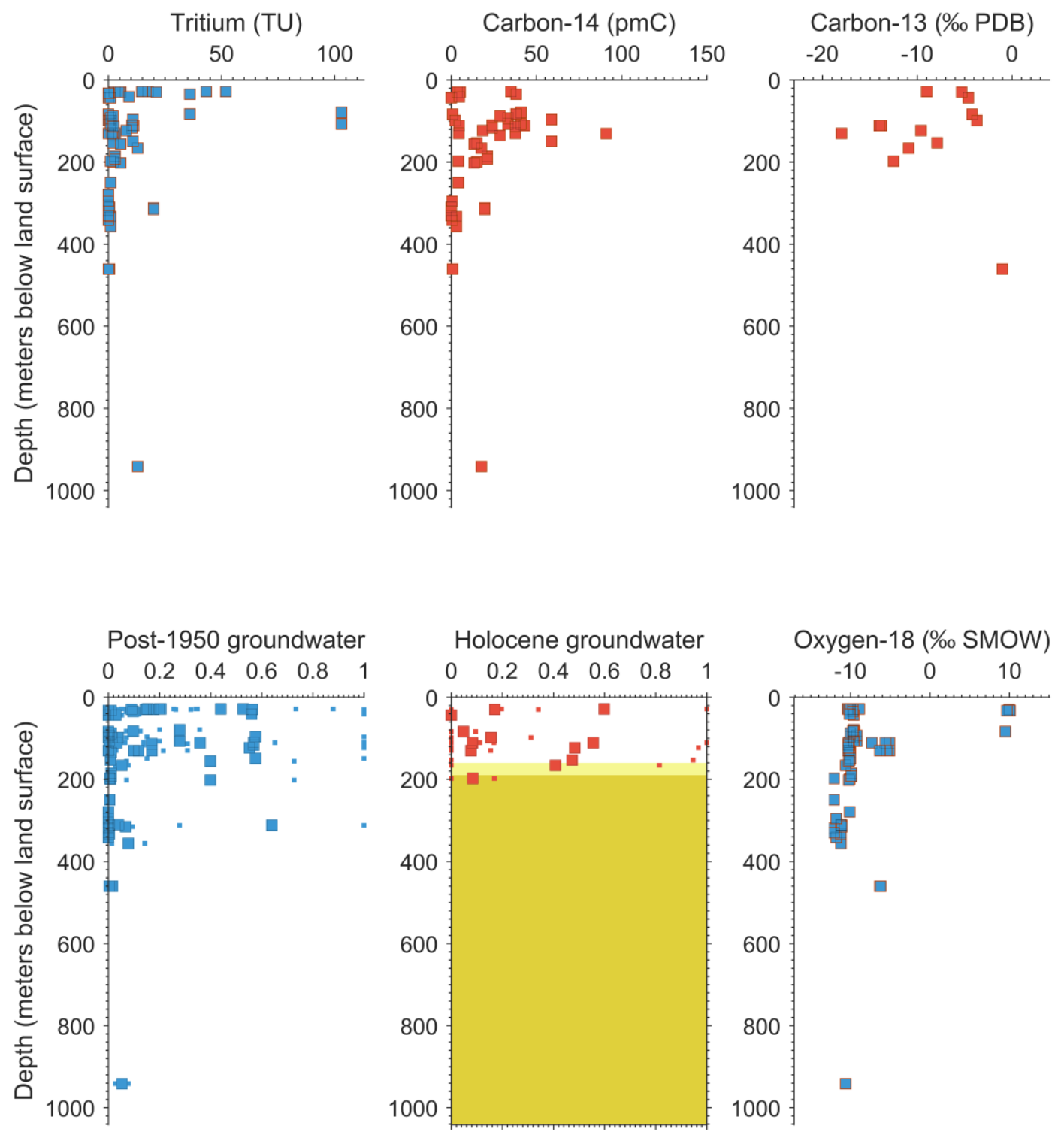

Southern Poland Carbonate Aquifers, Poland 

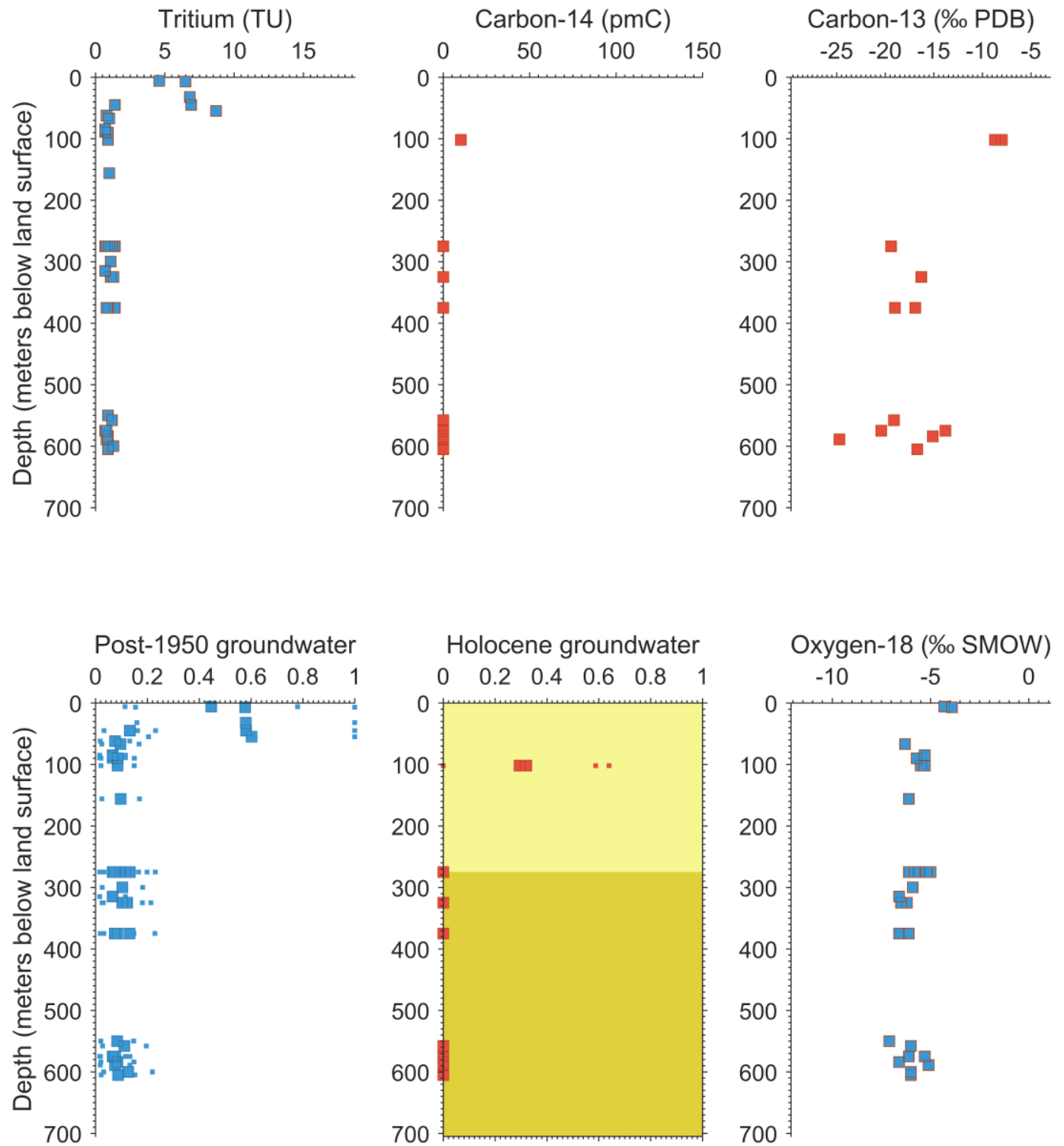

Chad Sedimentary basin (western), Nigeria 

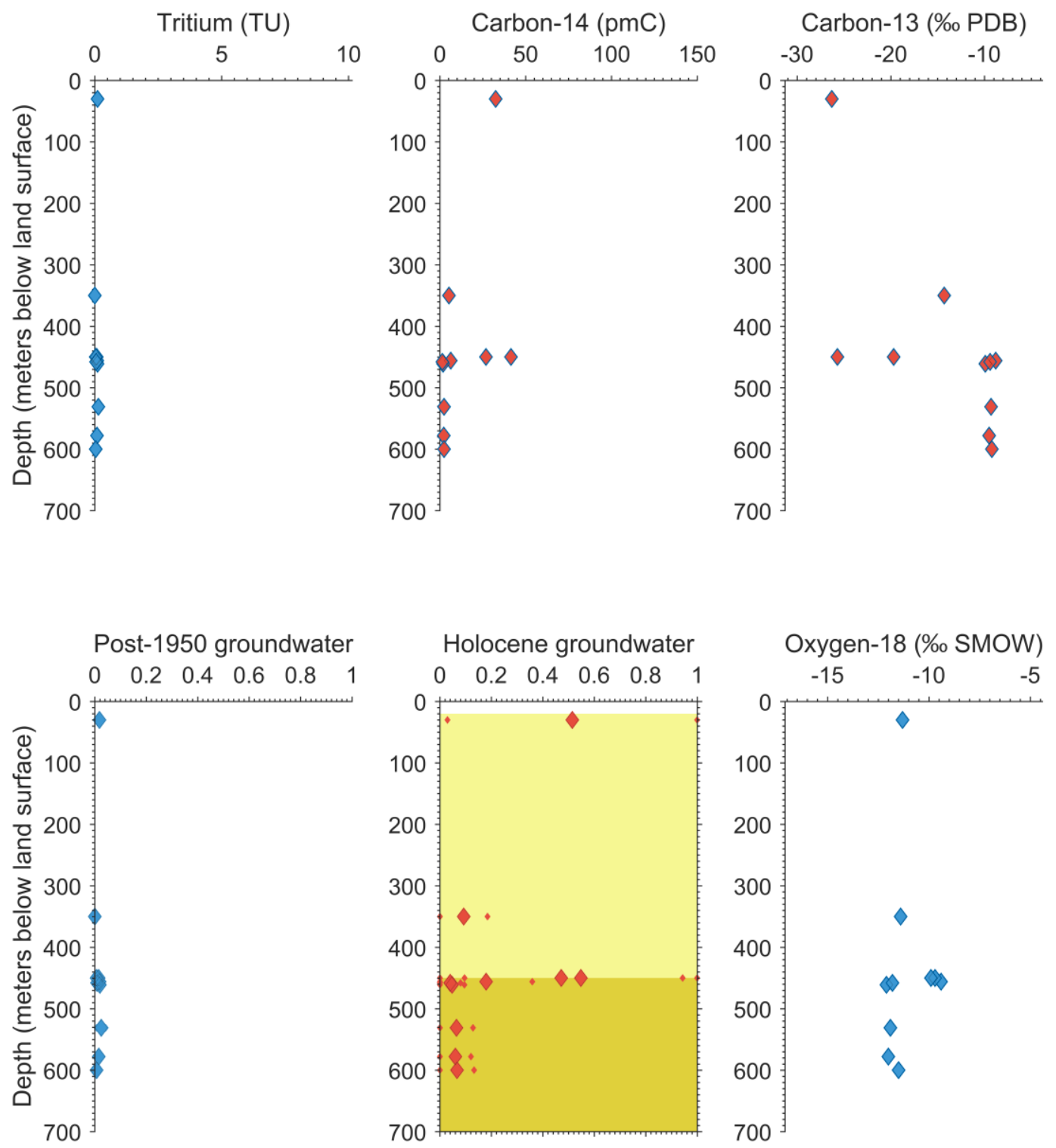

Kufra Basin, Libya 

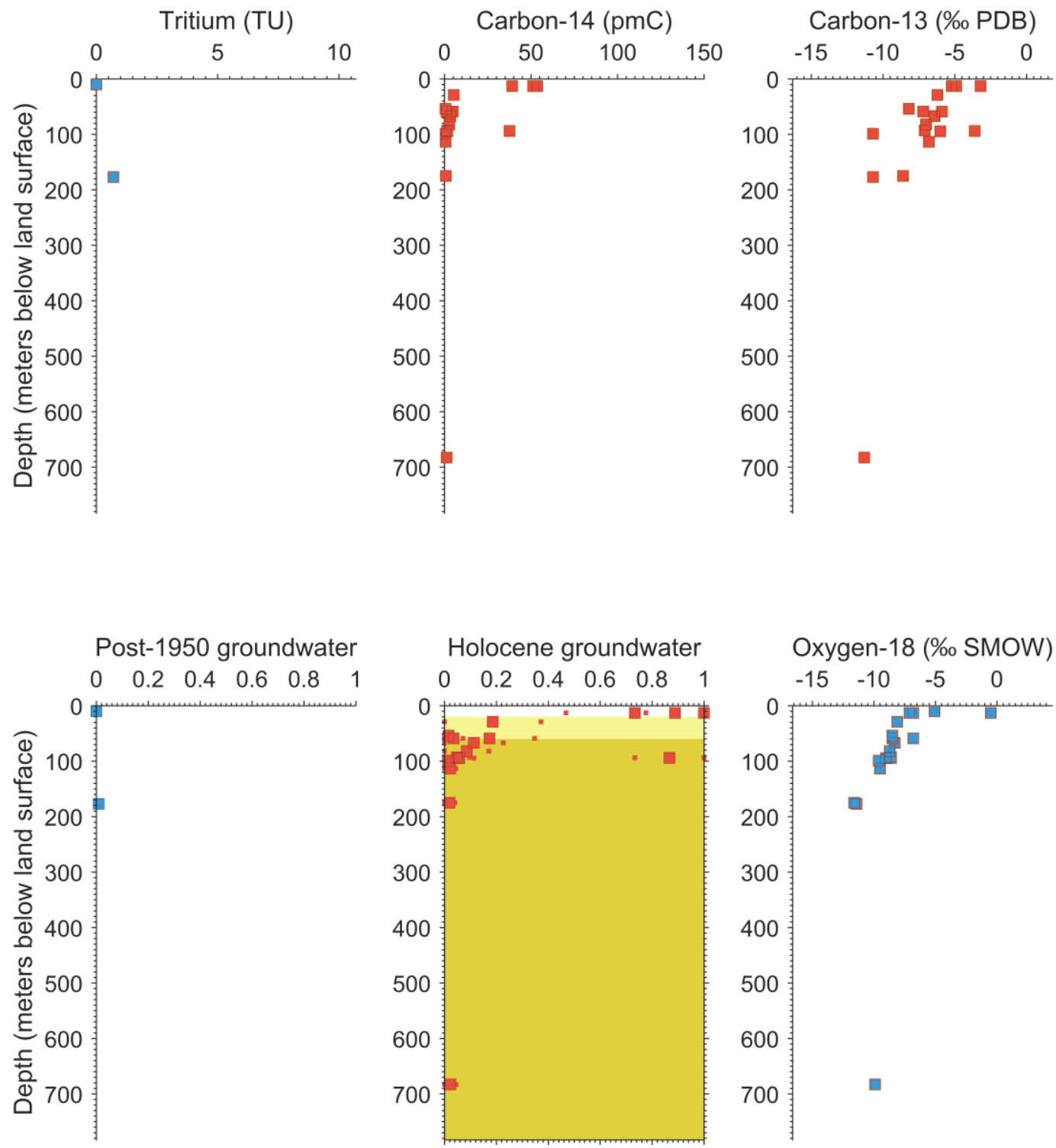

Sirte basin: N Libya, Libya 

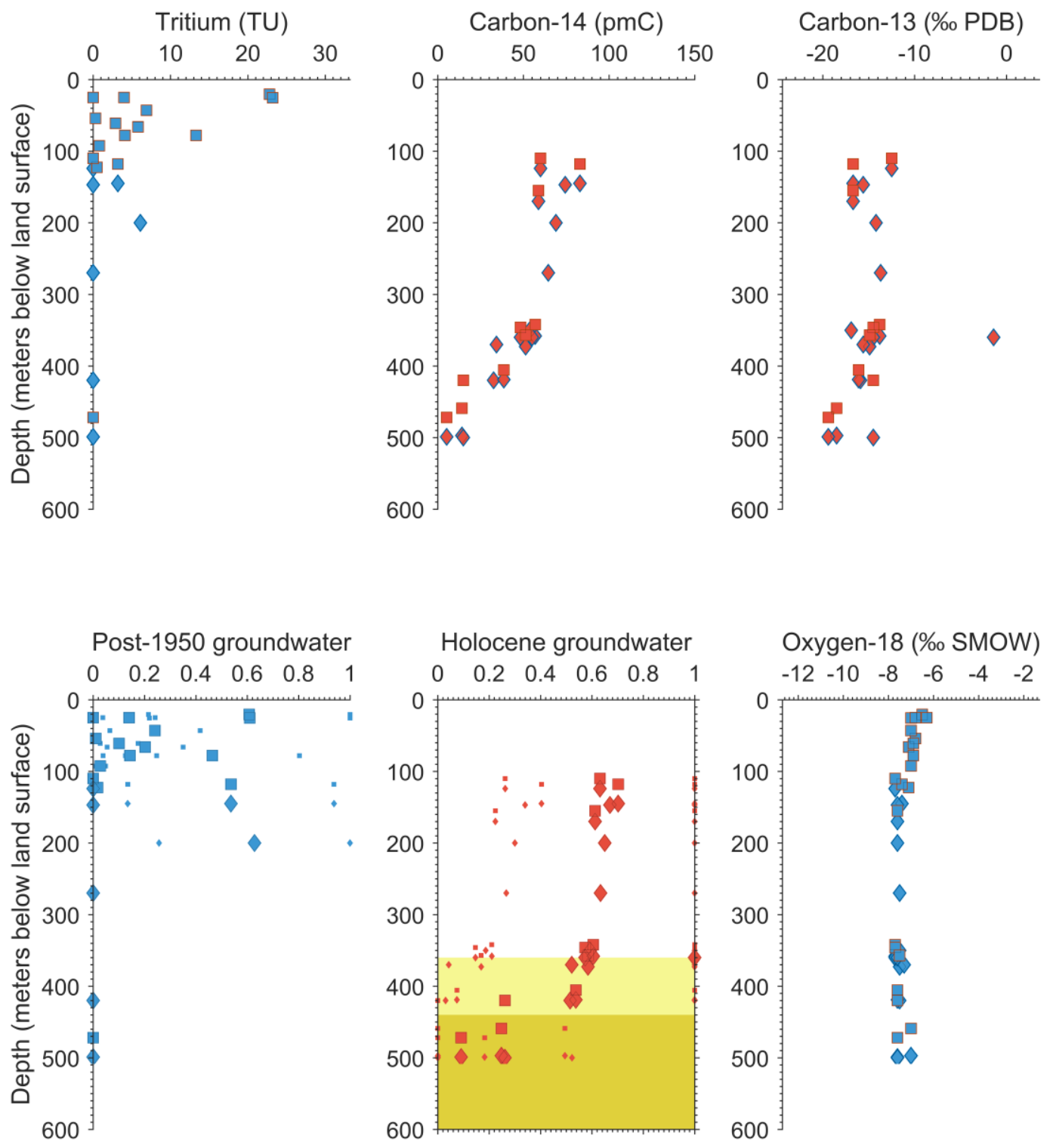

Kimitsu aquifer and Yoro River Basin, Japan 

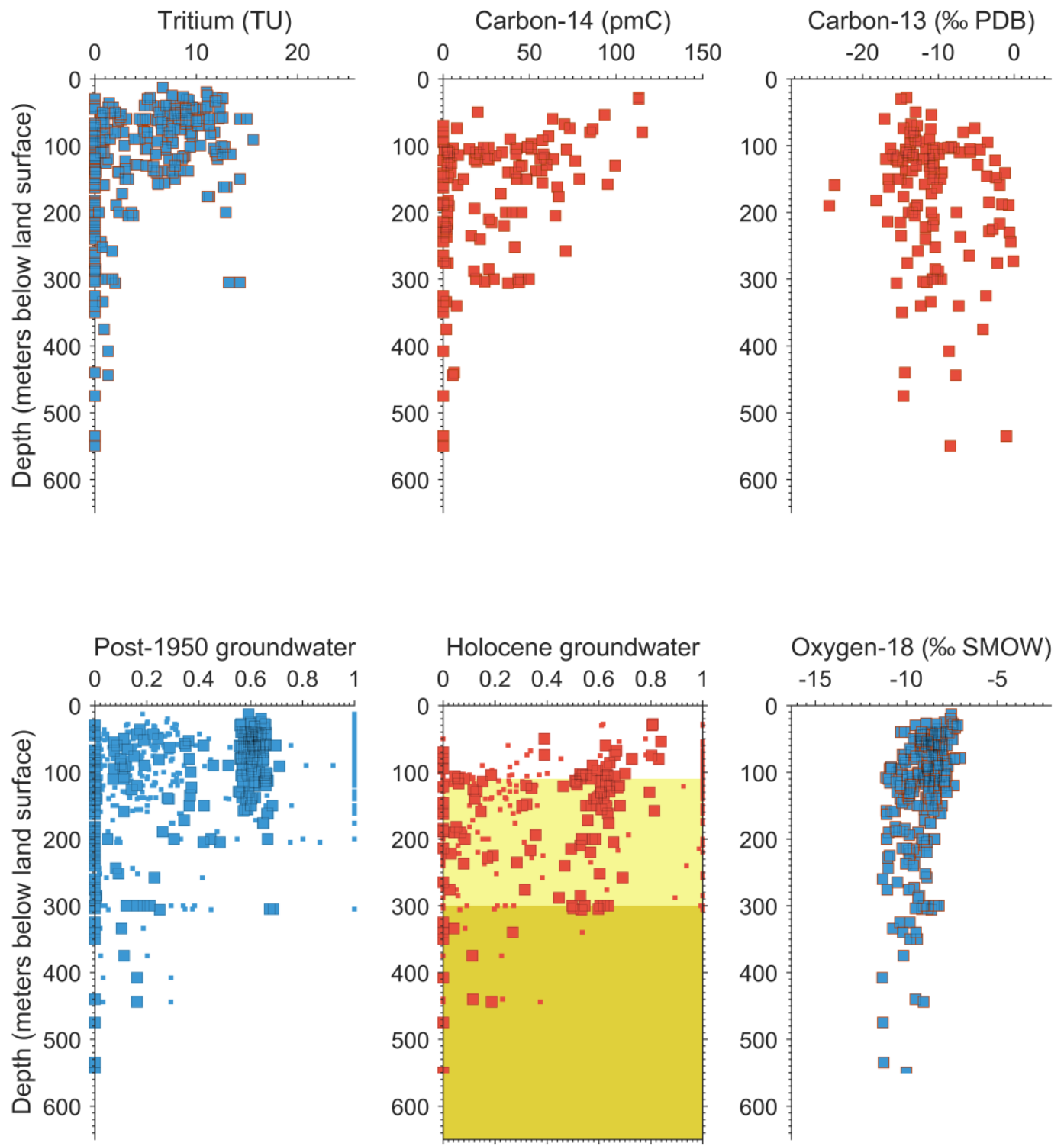

Emilia Romagna plain, Italy 

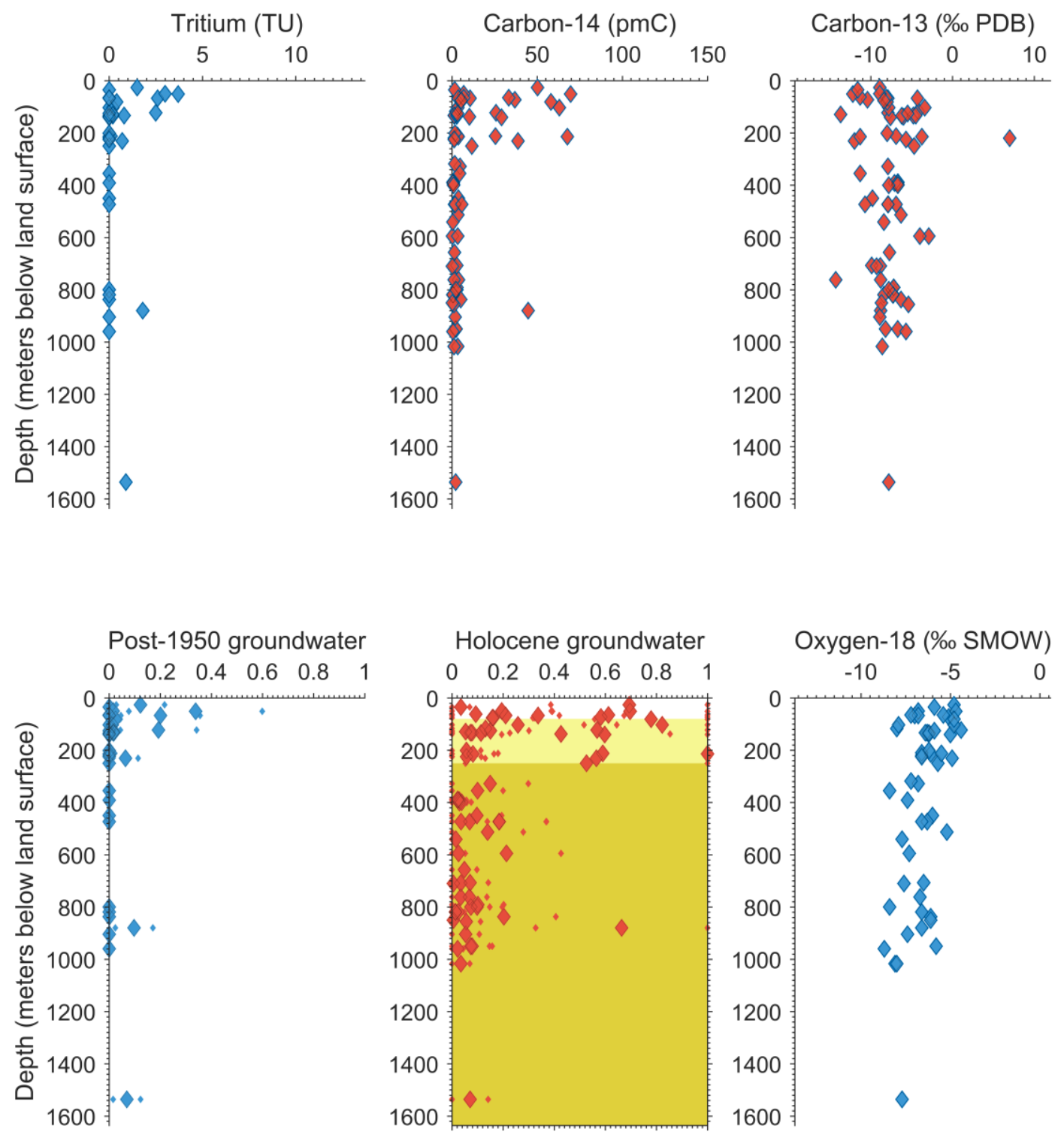

Arava Valley, Israel 

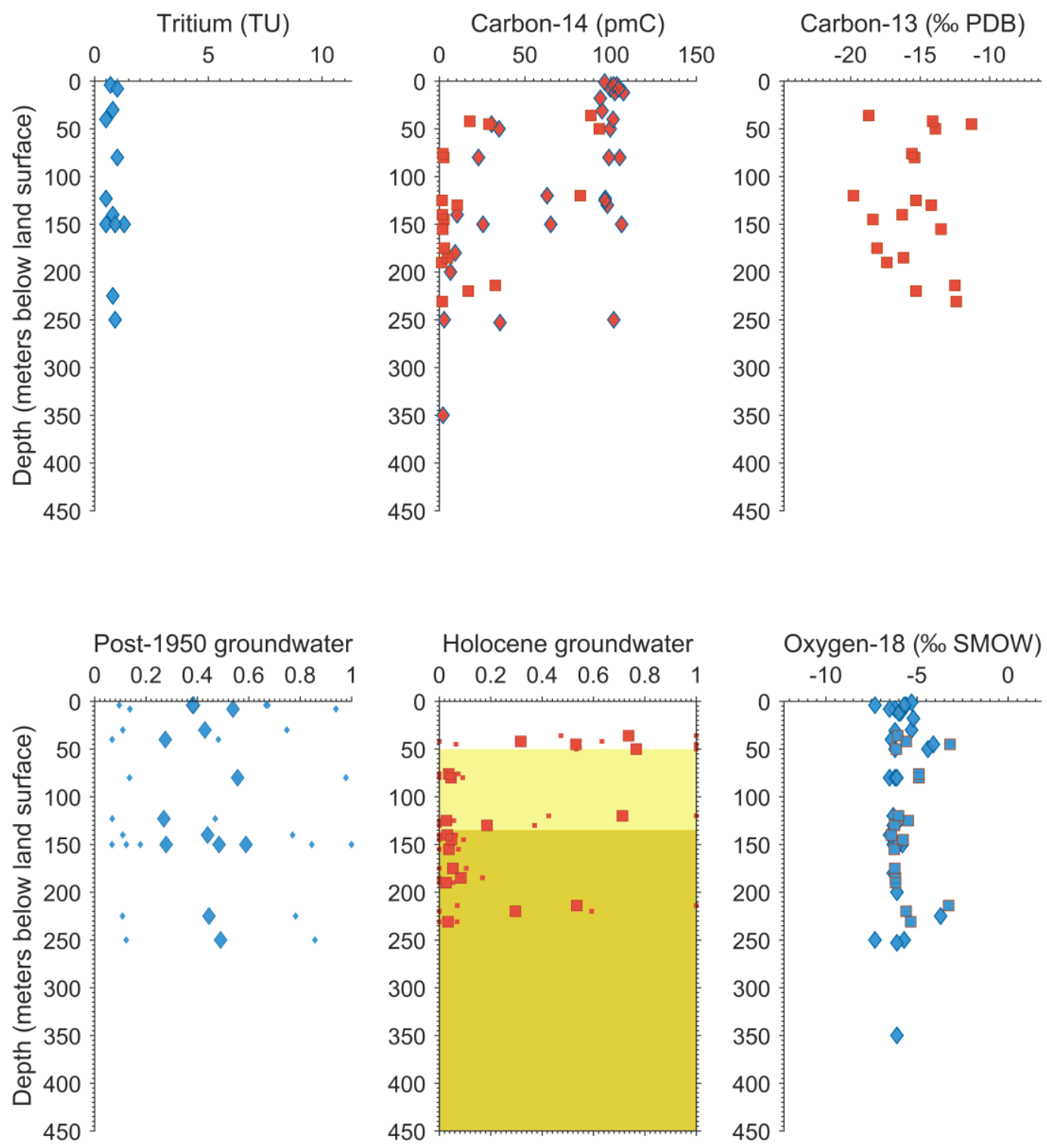

Jakarta basin aquifer, Indonesia 

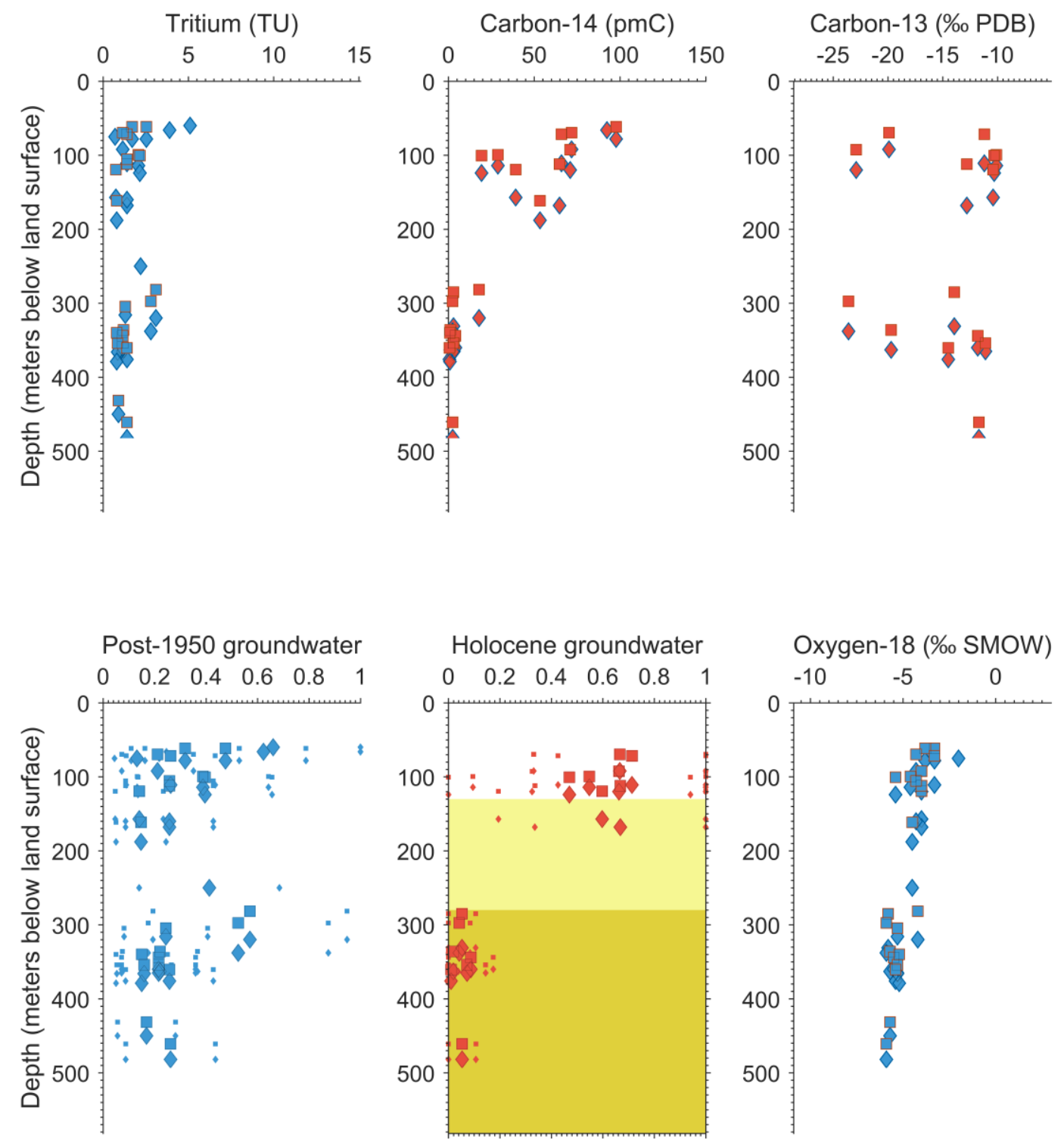

Tiruvadanai aquifer, India 

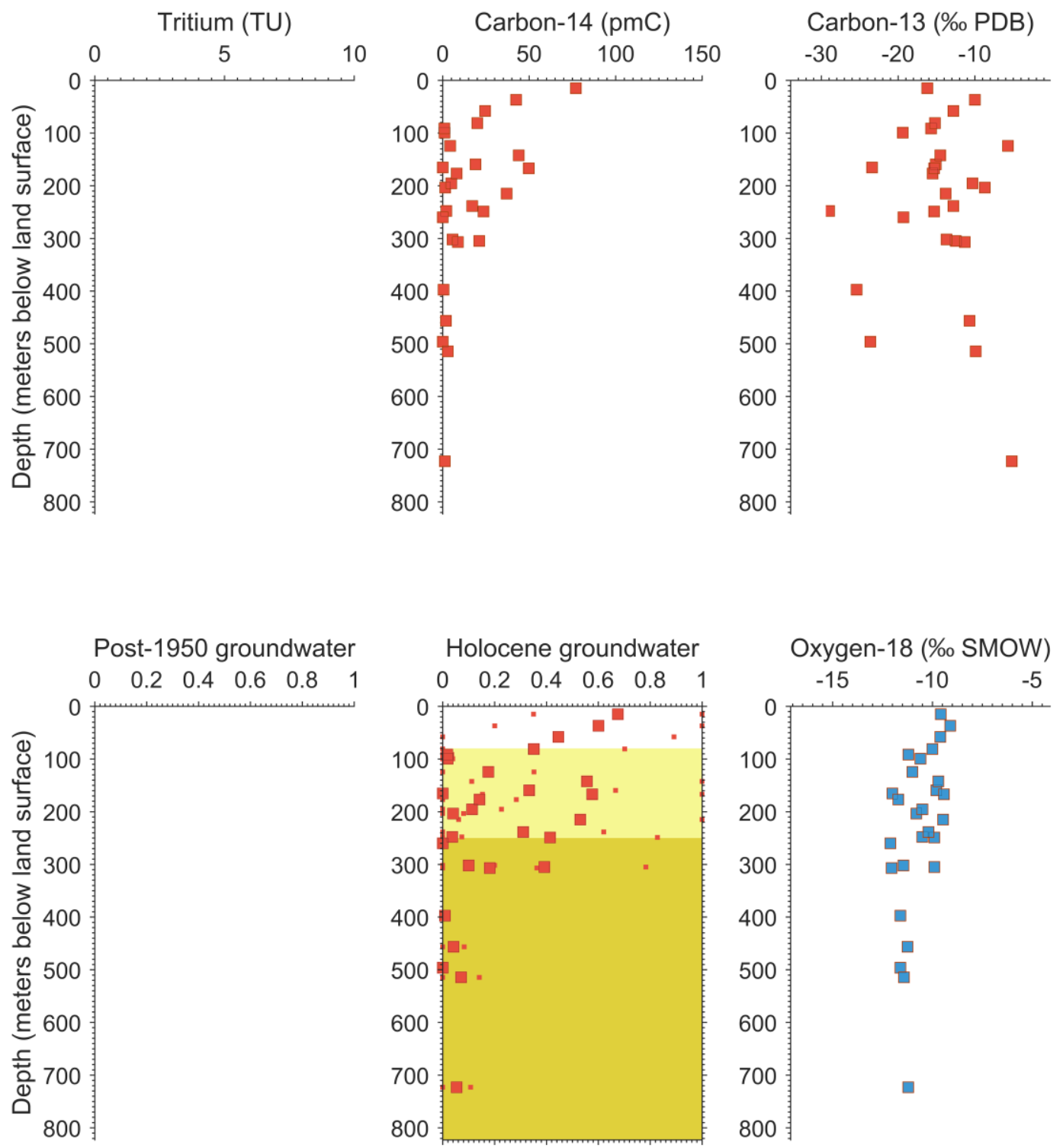

Great Hungarian Plain: Shallow unconfined, Hungary 

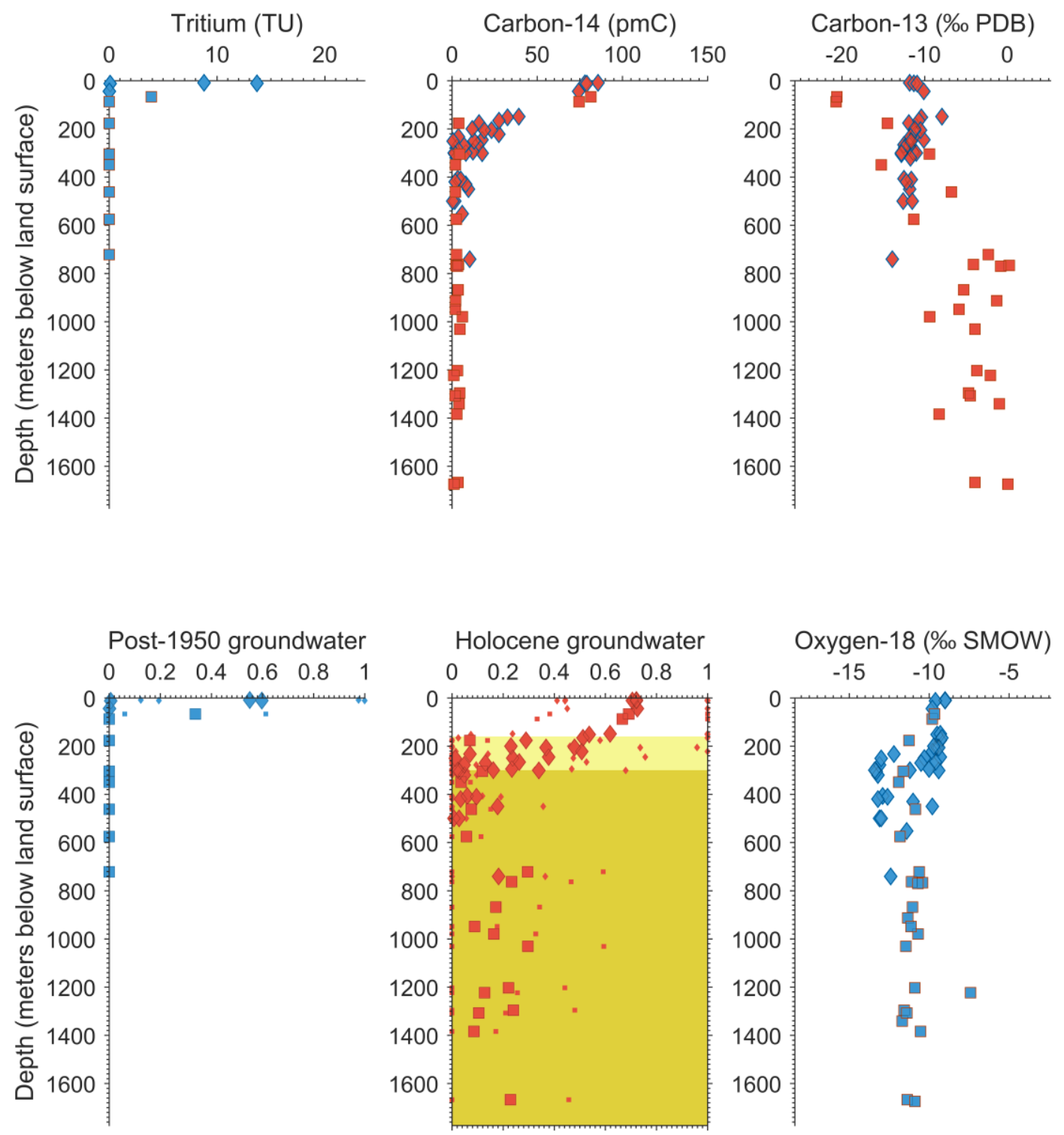

Pannonian Basin, Hungary 

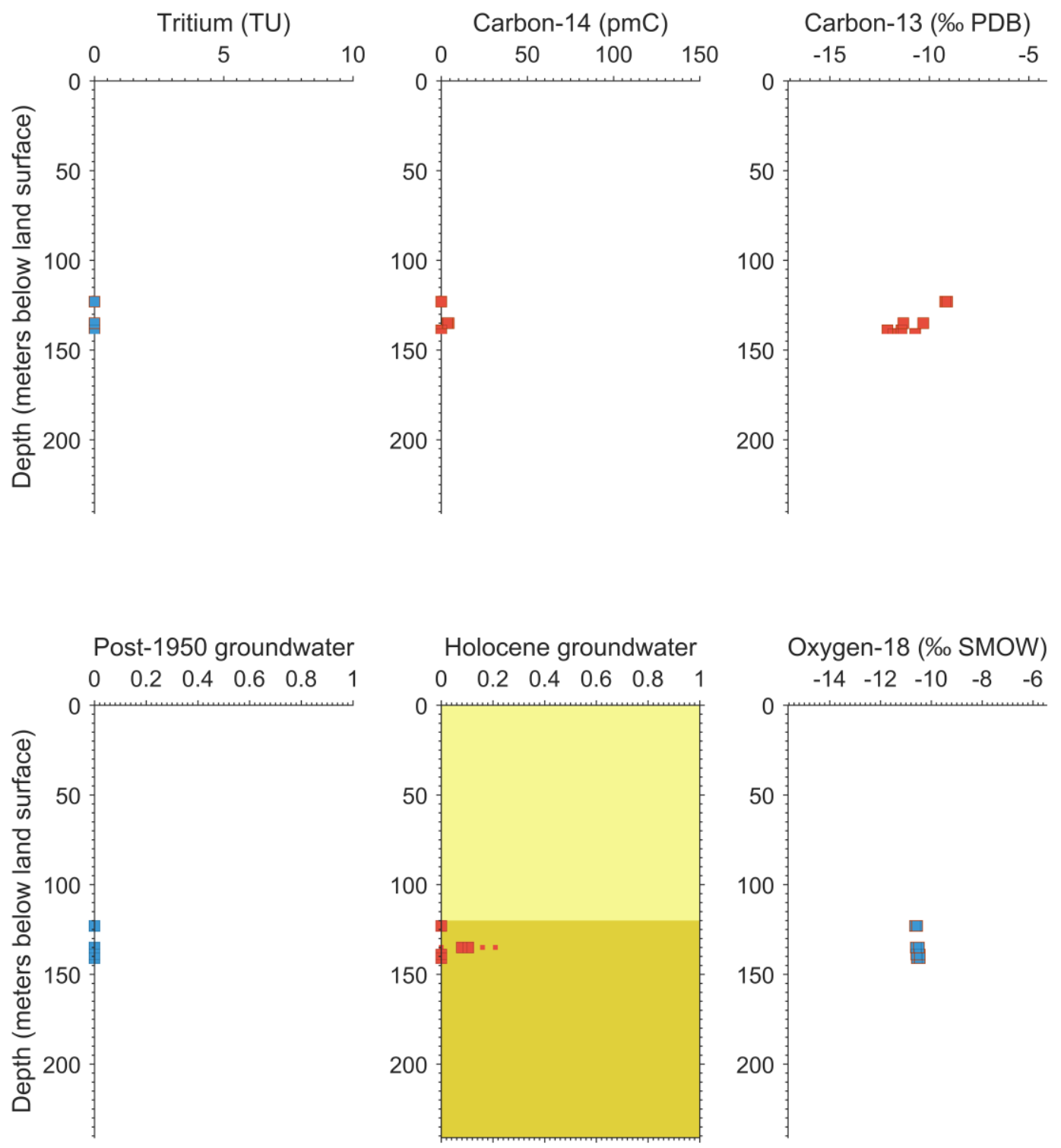

Benkerstein, Germany 

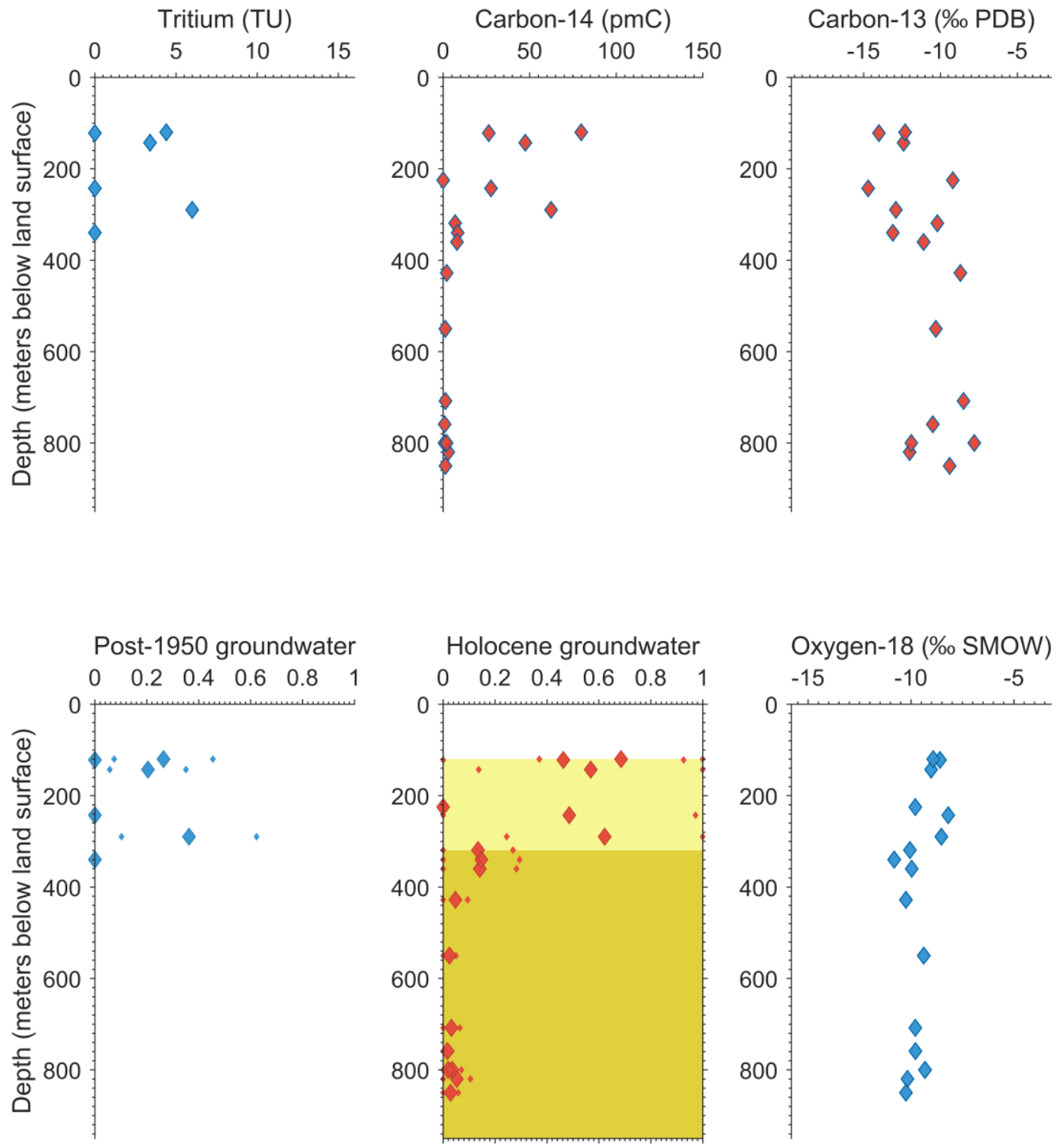

Lorraine Sandstone Aquifer, France 

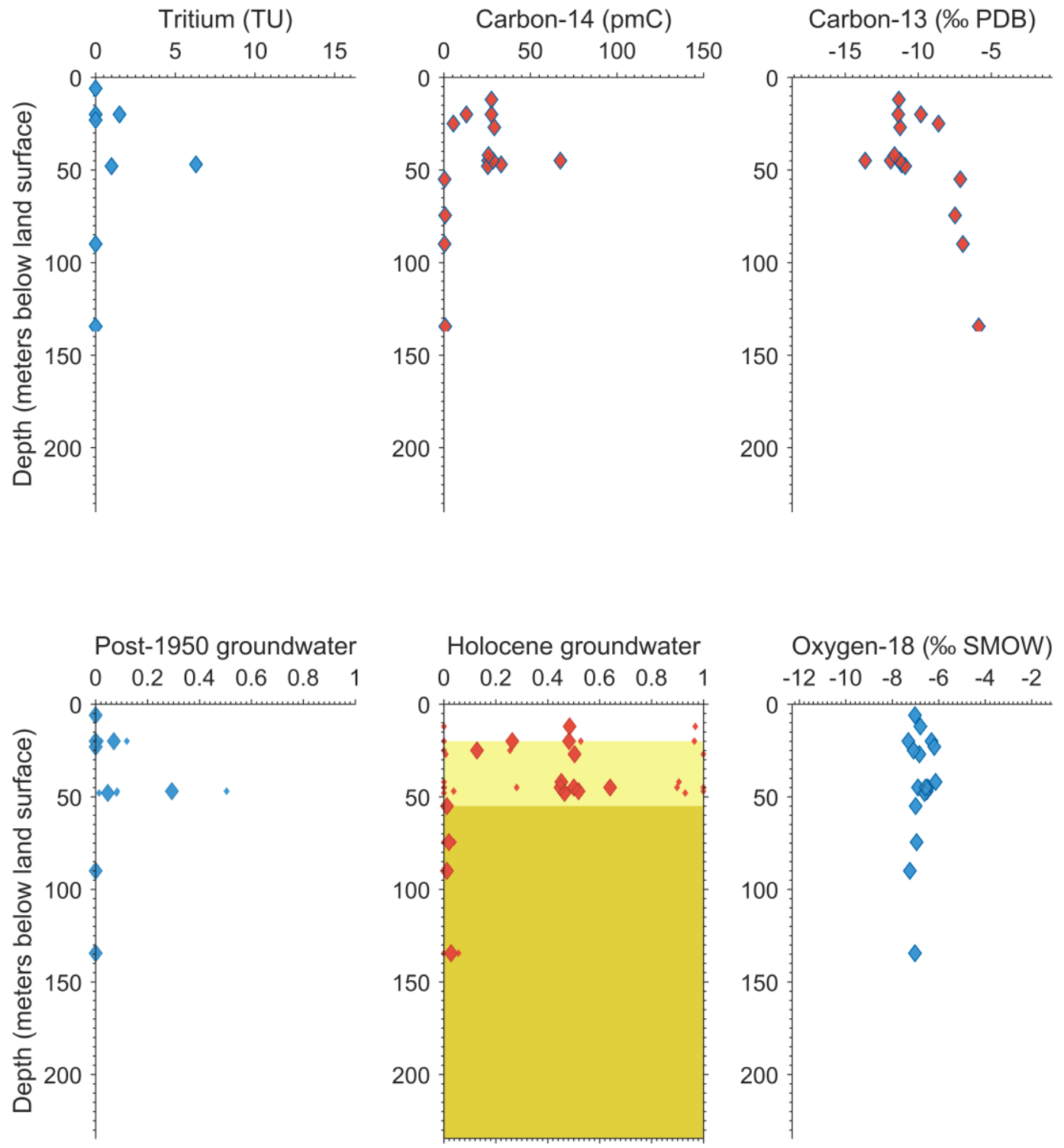

Bathonian and Bajocian coastal aquifer, France 

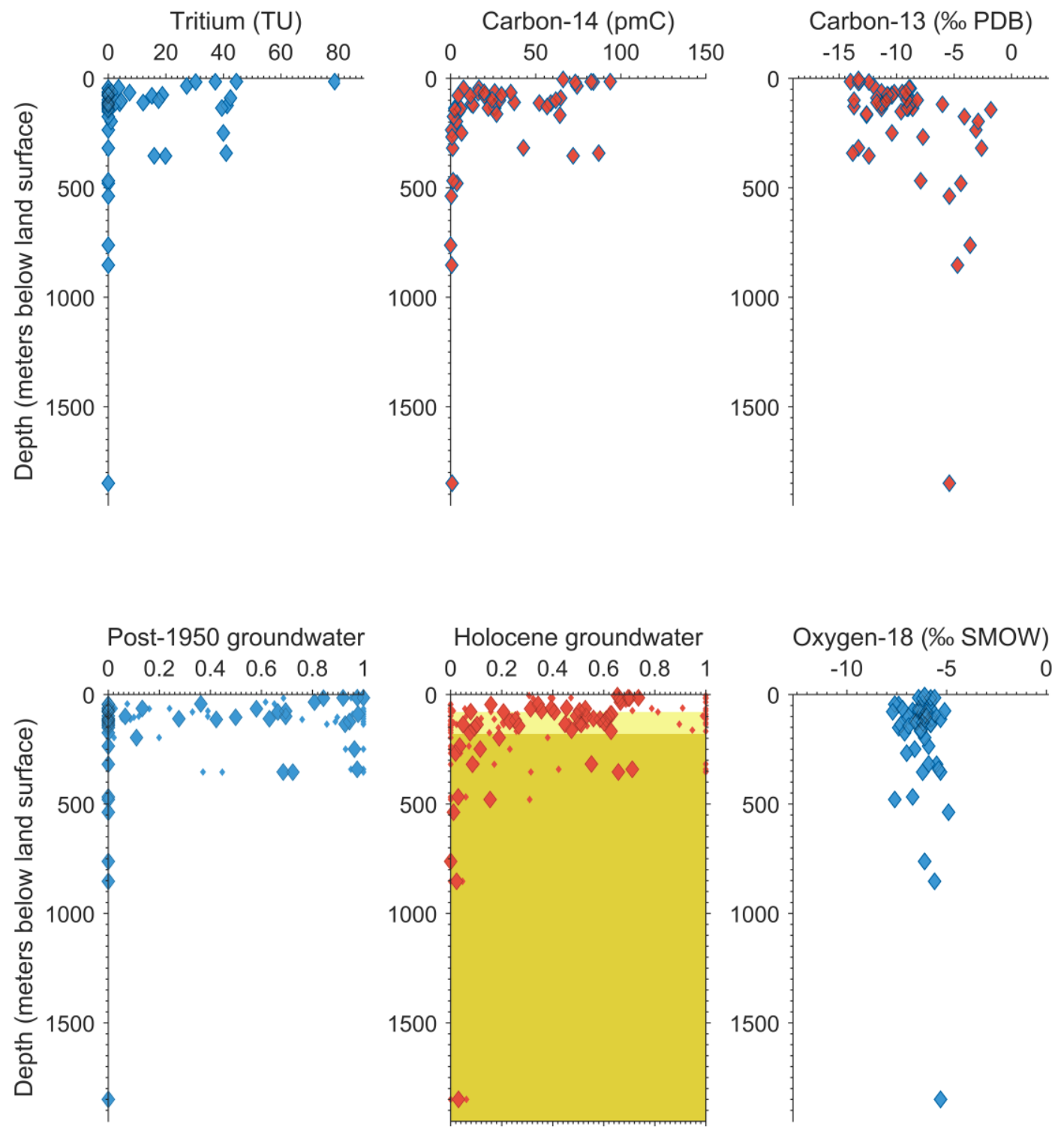

Aquitaine Basin, France 

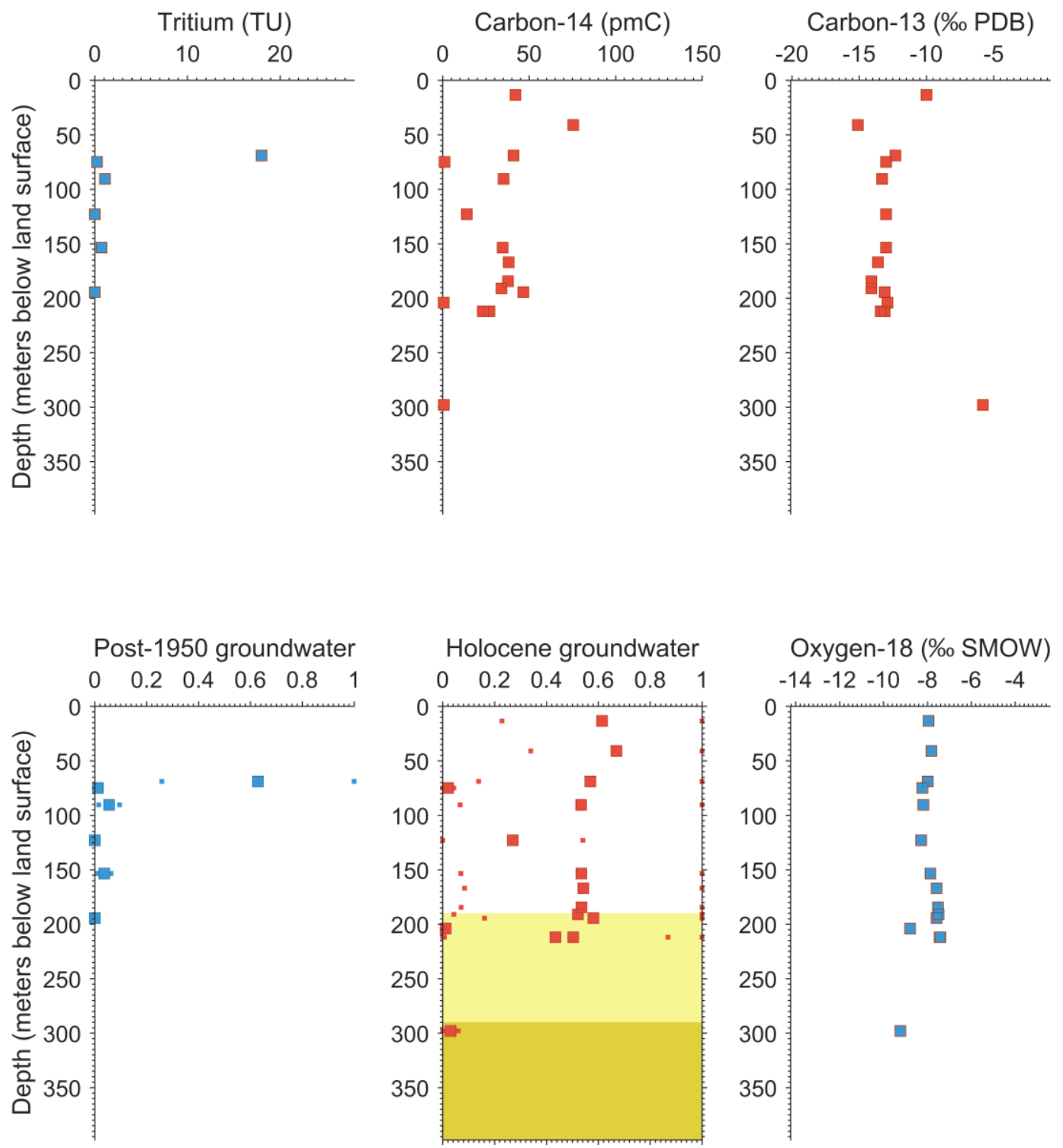

Ribe Formation, Denmark 

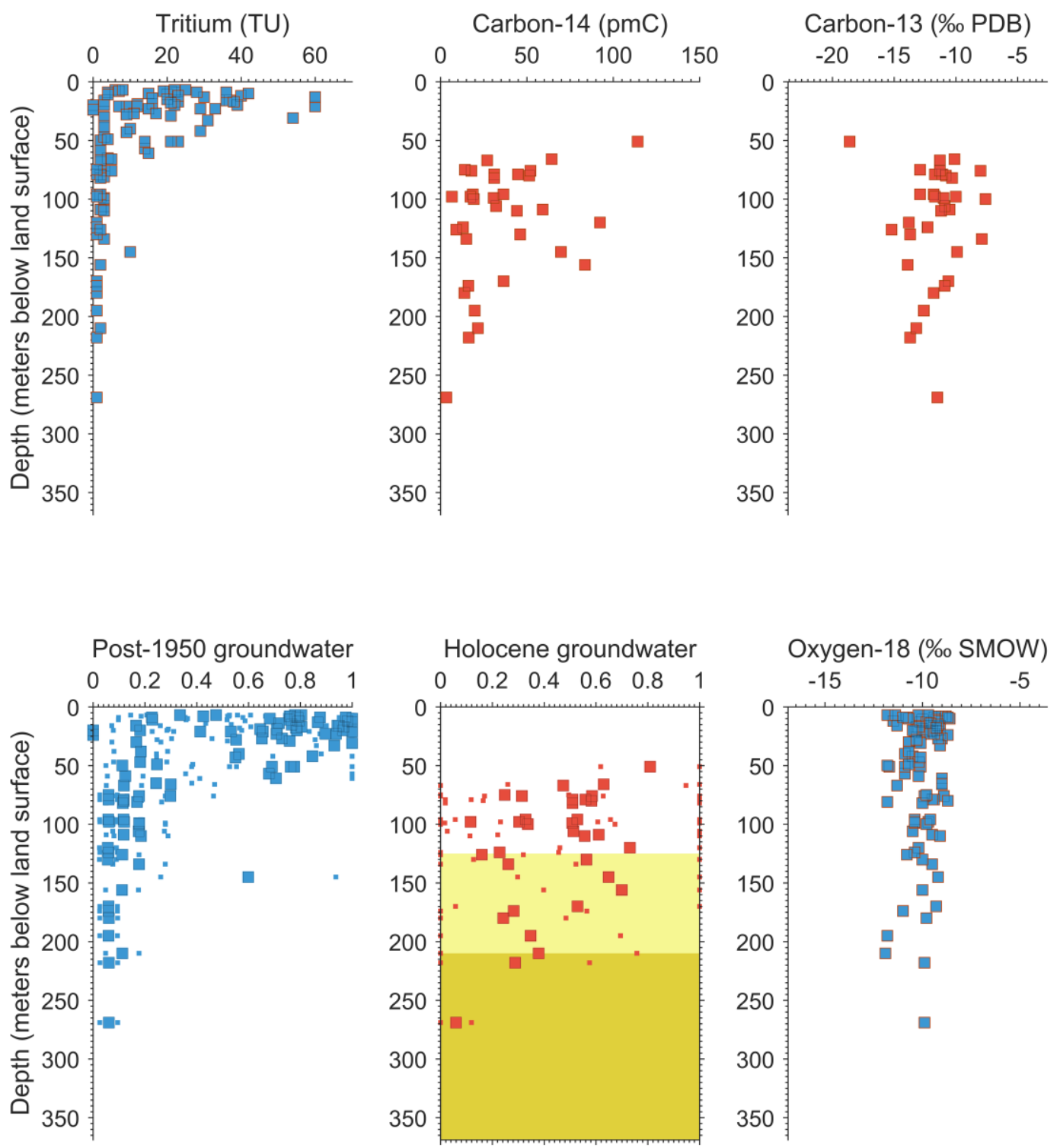

Songnen Plain aquifer, China 

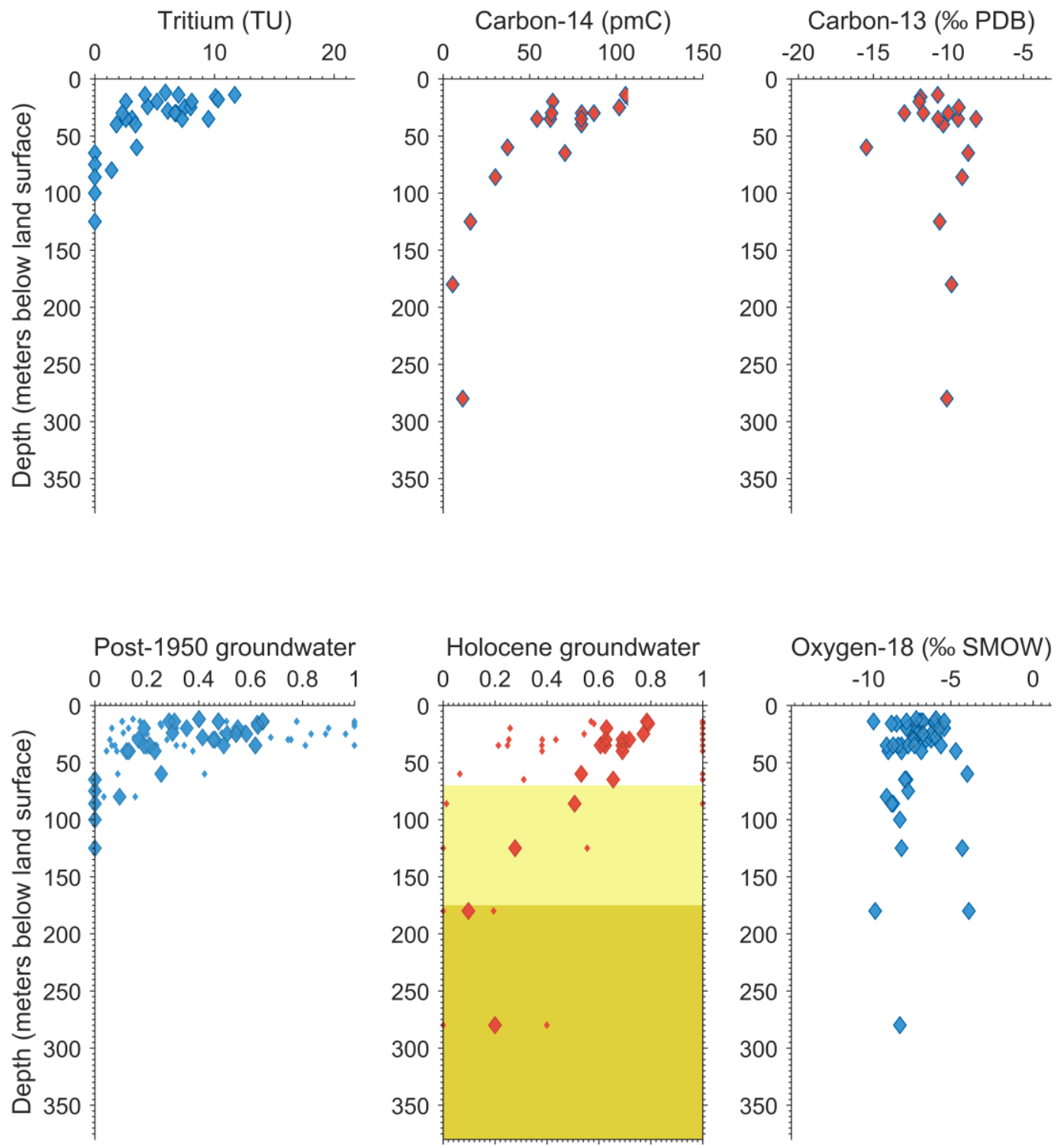

Laizhou Bay, China 

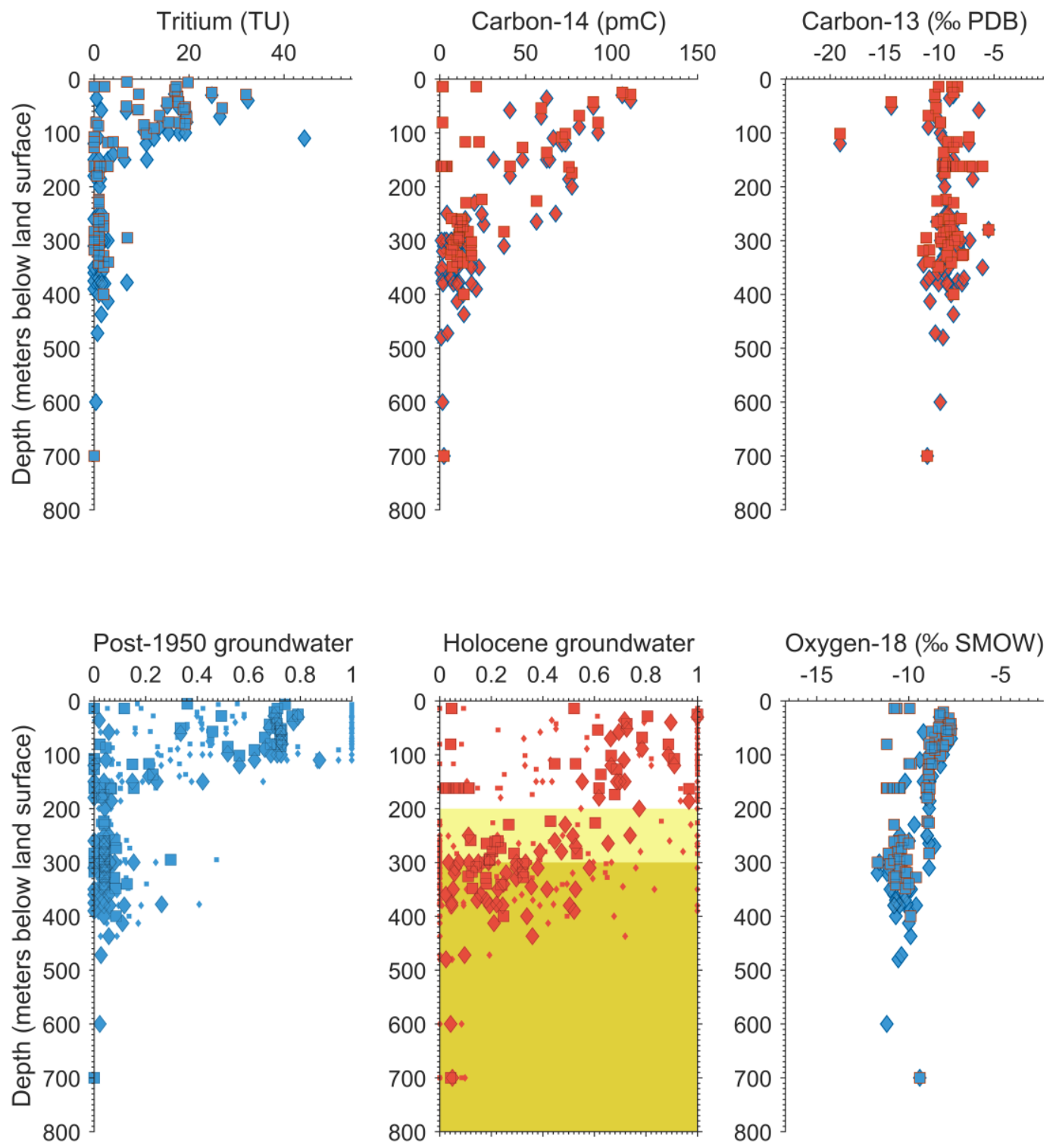

North China Plain, China 

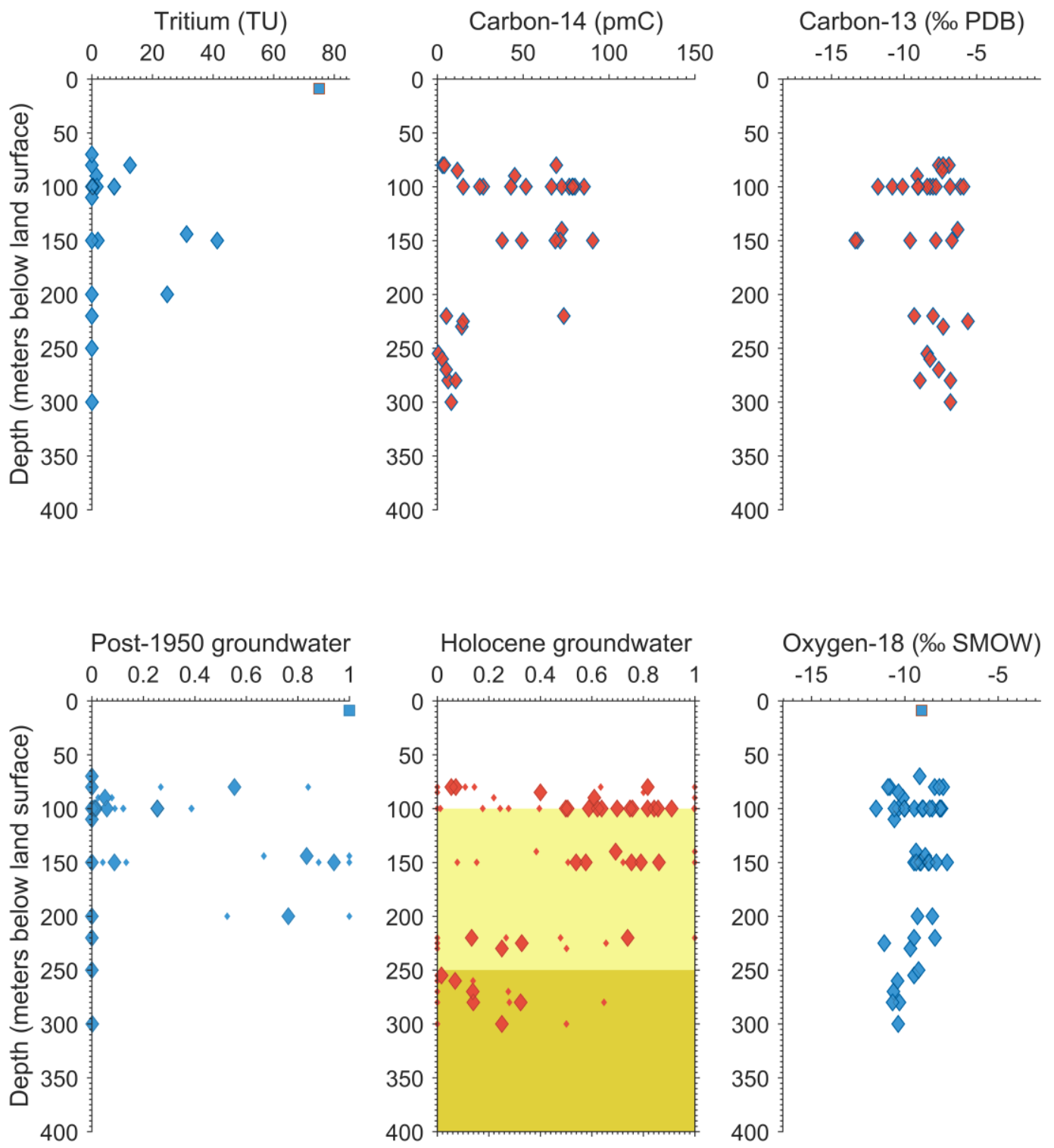

\section{Eastern Hexi Corridor, China}



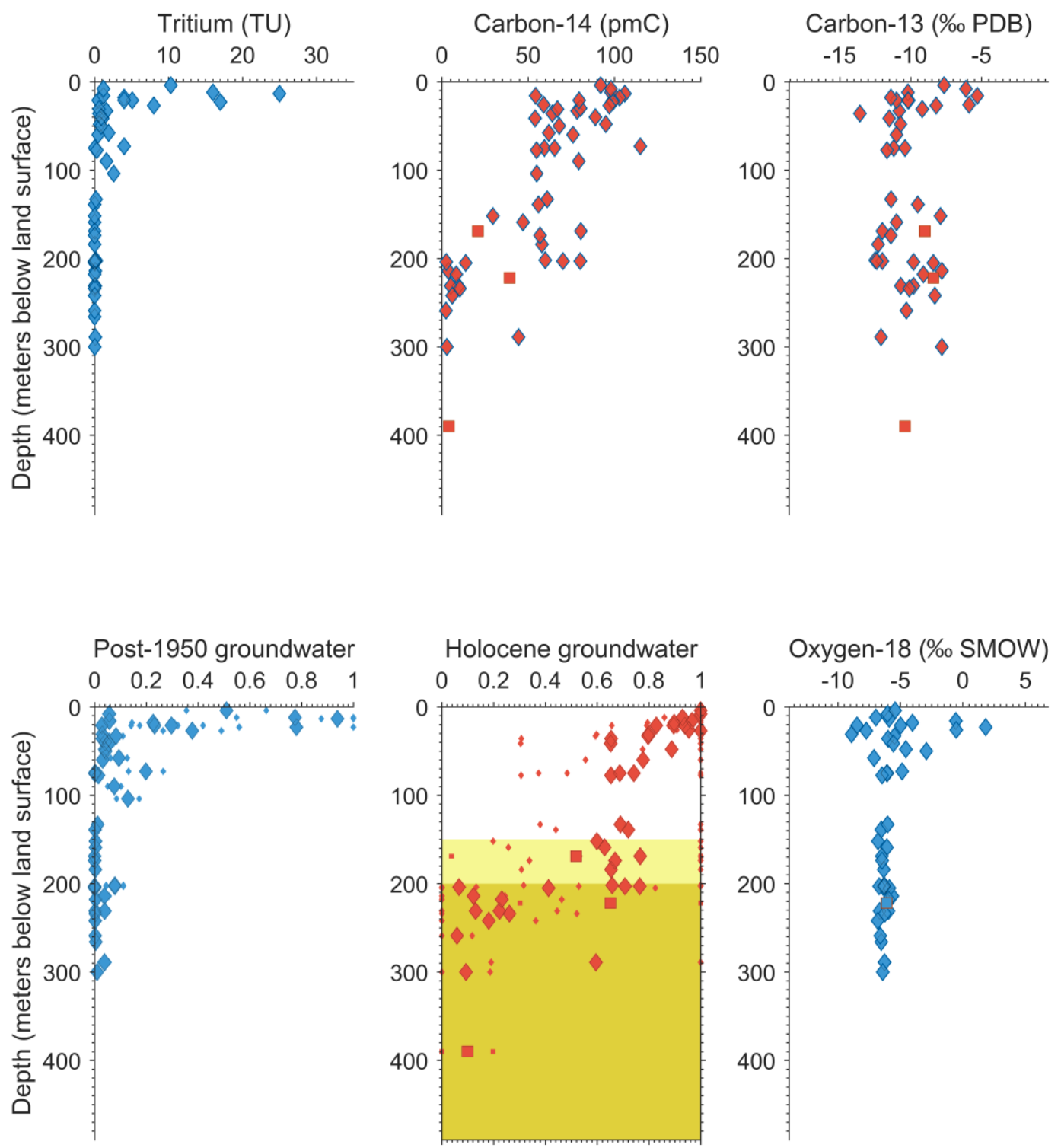

Kalahari Desert: Ntane Sandstone, Botswana 

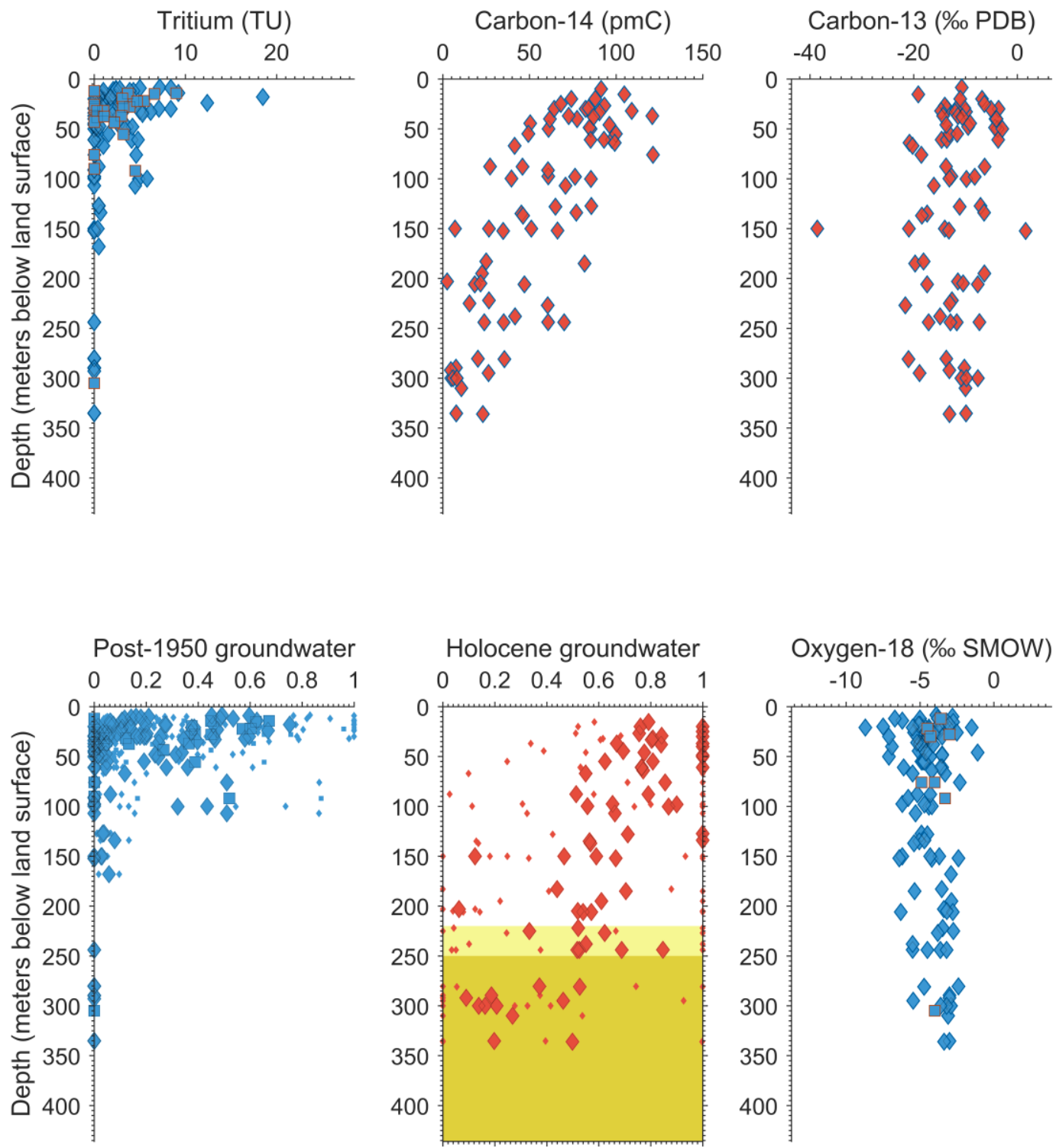

\section{Bengal Basin, Bangladesh}



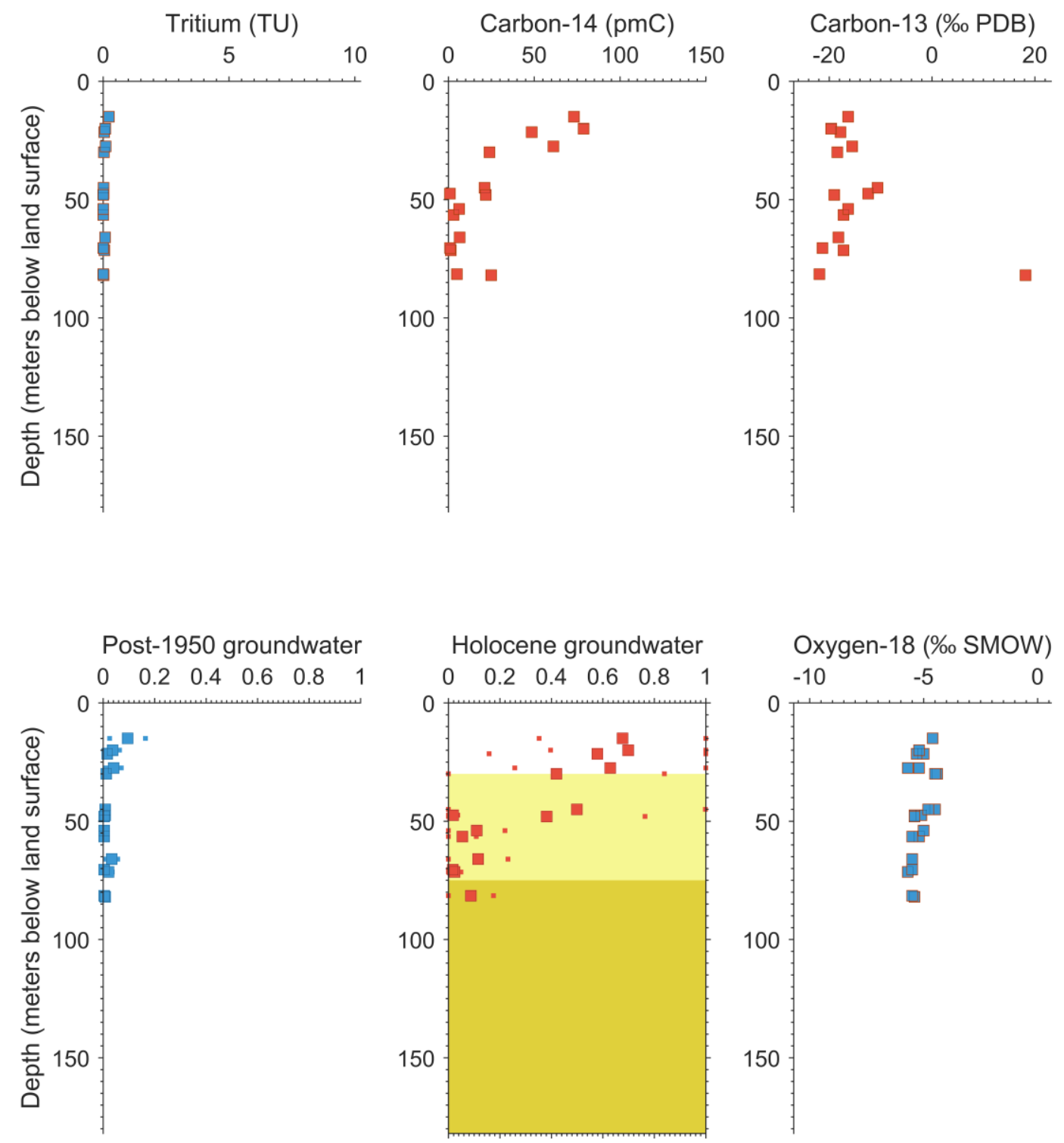

Western Port Basin, Australia 

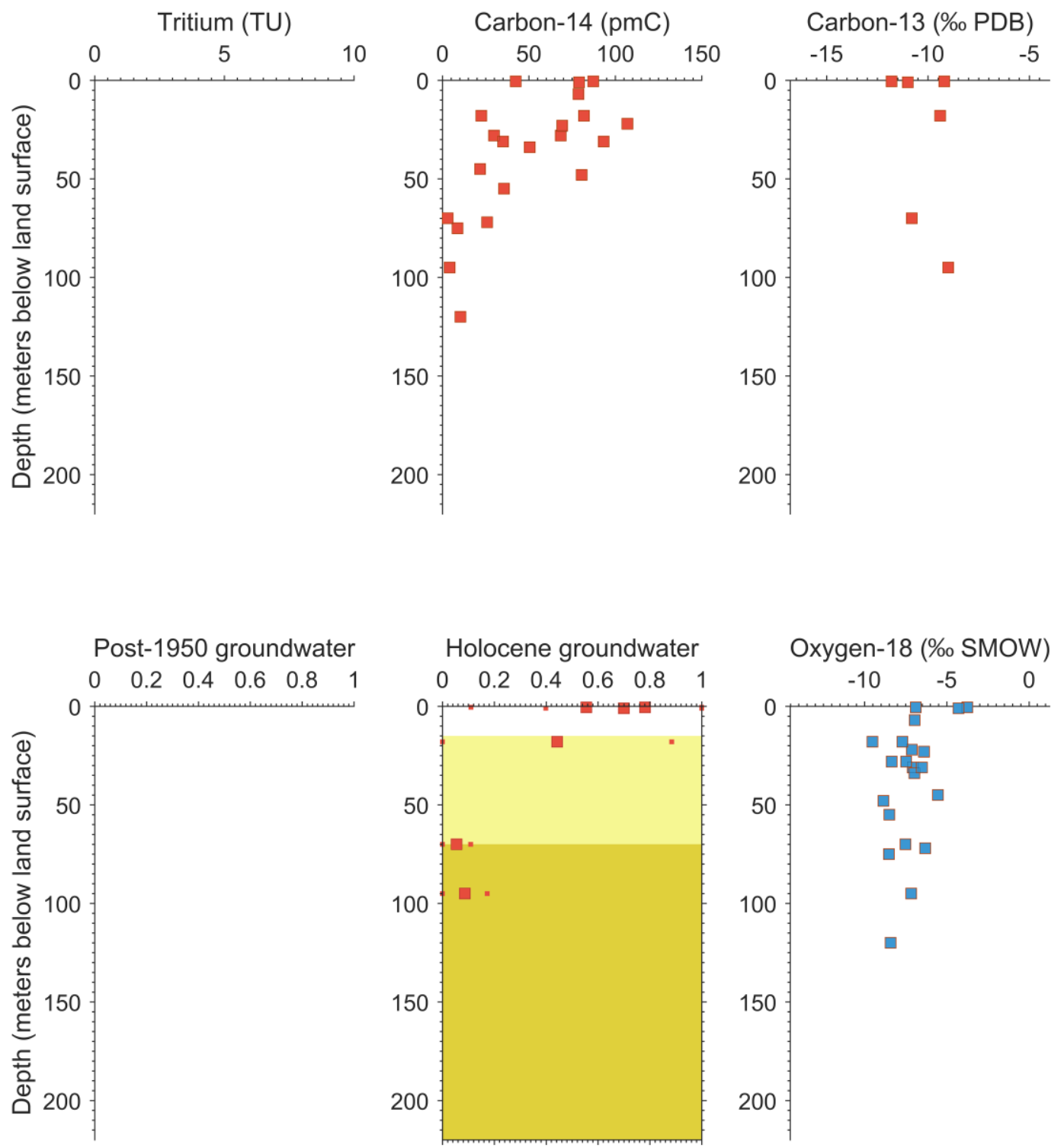

Ngalia and Amadeus Basin, Australia 